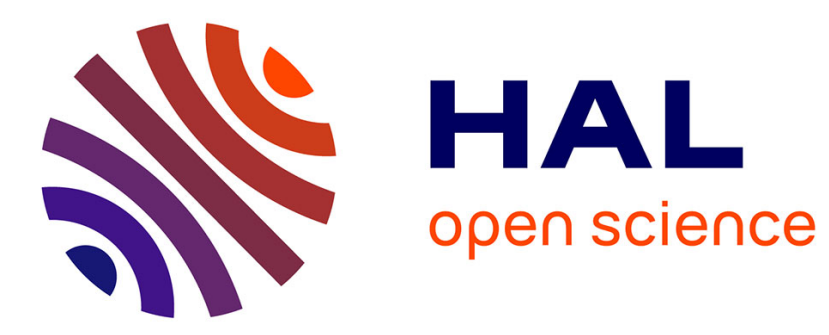

\title{
Analytical approximations of local-Heston volatility model and error analysis
}

Romain Bompis, Emmanuel Gobet

\section{To cite this version:}

Romain Bompis, Emmanuel Gobet. Analytical approximations of local-Heston volatility model and error analysis. 2015. hal-00839650v2

\section{HAL Id: hal-00839650 https://hal.science/hal-00839650v2}

Preprint submitted on 18 Mar 2015

HAL is a multi-disciplinary open access archive for the deposit and dissemination of scientific research documents, whether they are published or not. The documents may come from teaching and research institutions in France or abroad, or from public or private research centers.
L'archive ouverte pluridisciplinaire $\mathbf{H A L}$, est destinée au dépôt et à la diffusion de documents scientifiques de niveau recherche, publiés ou non, émanant des établissements d'enseignement et de recherche français ou étrangers, des laboratoires publics ou privés. 


\title{
ANALYTICAL APPROXIMATIONS OF LOCAL-HESTON VOLATILITY MODEL AND ERROR ANALYSIS*
}

\author{
R. BOMPIS ${ }^{\dagger}$ AND E. GOBET
}

\begin{abstract}
This paper consists in providing and mathematically analyzing the expansion of an option price (with bounded Lipschitz payoff) for model combining local and stochastic volatility. The local volatility part has a general form, with appropriate growth and boundedness assumptions. For the stochastic part, we choose a square root process, which is widely used for modeling the behavior of the variance process (Heston model). We rigorously establish tight error estimates of our expansions, using Malliavin calculus, which requires a careful treatment because of the lack of weak differentiability of the model; this error analysis is interesting on its own. Moreover, in the particular case of Call-Put options, we also provide expansions of the Black-Scholes implied volatility which allows to obtain very simple and rapid formulas in comparison to the Monte Carlo approach while maintaining a very competitive accuracy.
\end{abstract}

This version: March 18, 2015.

Key words. Analytical approximations; Local and stochastic volatilities; Malliavin calculus.

AMS subject classifications. $34 \mathrm{E} 10 ; 60 \mathrm{Hxx}$

\section{Introduction.}

$\triangleright$ Formulation of the problem. We aim at providing analytical approximations of

$$
\mathbb{E}\left[h\left(X_{T}\right)\right]
$$

for a bounded Lipschitz function $h: \mathbb{R} \mapsto \mathbb{R}$, a fixed maturity $T>0$, where $X$ is modeling the log-price of an asset, which dynamics are

$$
\begin{aligned}
\mathrm{d} X_{t} & =\sigma\left(t, X_{t}\right) \sqrt{V_{t}} \mathrm{~d} W_{t}-\frac{1}{2} \sigma^{2}\left(t, X_{t}\right) V_{t} \mathrm{~d} t, \quad X_{0}=x_{0} \in \mathbb{R}, \\
\mathrm{d} V_{t} & =\alpha_{t} \mathrm{~d} t+\xi_{t} \sqrt{V_{t}} \mathrm{~d} B_{t}, \quad V_{0}=v_{0}>0, \\
\mathrm{~d}\langle W, B\rangle_{t} & =\rho_{t} \mathrm{~d} t,
\end{aligned}
$$

where $\left(W_{t}, B_{t}\right)_{0 \leq t \leq T}$ is a two-dimensional correlated Brownian motion defined on a filtered probability space $\left(\Omega, \mathcal{F},\left(\mathcal{F}_{t}\right)_{0 \leq t \leq T}, \mathbb{P}\right)$ with the usual assumptions on the filtration. This problem is motivated by option pricing: in this framework, asset and option prices are forward prices or equivalently, interest-rates and dividends are set to 0 . In the model $(1.2)-(1.3), \sigma(.,$.$) is the time-$ dependent local volatility function, $\left(V_{t}\right)_{t \in[0, T]}$ is a square root process (a.k.a. CIR process), which models the stochastic variance; the non-negative drift $\left(\alpha_{t}\right)_{t}$, the non-negative volatility of volatility $\left(\xi_{t}\right)_{t}$ and the correlation $\left(\rho_{t}\right)_{t}$ are bounded measurable functions of time. Because the stochastic variance (1.3) is of the Heston form, we call this model local-Heston volatility model. Actually, our parametrization is equivalent to the usual "mean-reverting" form $\mathrm{d} \tilde{V}_{t}=\left(\alpha_{t}-\kappa_{t} \tilde{V}_{t}\right) \mathrm{d} t+\xi_{t} \sqrt{\tilde{V}_{t}} \mathrm{~d} B_{t}$

\footnotetext{
*A preliminary version has circulated as a working paper under the title "Price expansion formulas for model combining local and stochastic volatility", http://hal.archives-ouvertes.fr/hal-00839650

${ }^{\dagger}$ Email: romain.bompis@yahoo.fr. CMAP, Ecole Polytechnique and CNRS, Route de Saclay, 91128 Palaiseau cedex, France.

‡Email: emmanuel.gobet@polytechnique.edu. CMAP, Ecole Polytechnique and CNRS, Route de Saclay, 91128 Palaiseau cedex, France. Corresponding author. This research is part of the Chair Financial Risks of the Risk Foundation and the FiME Laboratory.
} 
(with deterministic $\left.\left(\kappa_{t}\right)_{t}\right)$ and $\mathrm{d} X_{t}=\sigma\left(t, X_{t}\right) \sqrt{\tilde{V}_{t}} \mathrm{~d} W_{t}-\frac{1}{2} \sigma^{2}\left(t, X_{t}\right) \tilde{V}_{t} \mathrm{~d} t$ : indeed by setting $V_{t}=$ $\tilde{V}_{t} \exp \left(\int_{0}^{t} \kappa_{s} \mathrm{~d} s\right)$, observe that $(X, V)$ solves a system of the form (1.2)-(1.3) with straightforward modifications of $\sigma, \xi, \alpha$. Our parametrization is more convenient for the subsequent analysis.

Models combining local and stochastic volatilities have emerged in the last decade to offer more flexibility in the skew and smile management: see for instance the well-known SABR model by Hagan etal. [HKLW02], the CEV-Heston model studied by Forde and Pogudin [FP13]. In view of the rather general form (1.2)-(1.3), the pricing (and thus the model calibration) is challenging, in particular because of the lack of closed-form formulas and because PDE or Monte-Carlo based numerical methods are too much time-consuming for real-time uses. In this work, we address this issue by deriving an expansion formula for $\mathbb{E}\left[h\left(X_{T}\right)\right]$ within the family of correlated local-Heston model. We do not argue that this is the best model to fit market data, nevertheless this is very popular among practitioners since it has the flavor to encompass the local volatility model and the Heston one. For works related to calibration, see [EKO11]. The interest of this work is not only the expansion formula but also the rigorous treatment of error estimates, an issue which is often not handled in the literature (sometimes computations are formal). In our case of square root process (little smooth), Lipschitz payoffs and pointwise ellipticity of local volatility, we develop a strategy of proof which is interesting on its own. The reader could argue that by smoothing data, expanding the modified $\mathbb{E}\left[h\left(X_{T}\right)\right]$ at a given order and then passing to the limit w.r.t. the smoothing parameter, we could avoid these technicalities. This may be incorrect: for instance in [FPSS04], this strategy applied to non-smooth data leads to a loss of theoretical accuracy (see the error estimates in [FPSS04, Theorem 3 and Lemma 1] in the context of call- option pricing). Even worse, in [GP14] about Backward Stochastic Differential Equations, the authors prove that the irregularity in the coefficients may discard any possibility of expansion at a given order. With these examples in mind, we believe that proving rigorous error estimates when coefficients/functions are not smooth is a serious mathematical issue which can not be avoided and which, once done, brings confidence in the derived expansion and sheds light on the needed assumptions.

$\triangleright$ Methodology. We follow the proxy approach initiated in [BGM09], by employing Malliavin calculus to compute expansion terms and to derive tight error estimates as a function of the most important model and payoff parameters (non-asymptotic error analysis). Inspired by [BGM09, BGM10b], we choose the following Gaussian proxy:

$$
\left\{\begin{array}{l}
\mathrm{d} X_{t}^{P}=\sigma\left(t, x_{0}\right) \sqrt{v_{t}} \mathrm{~d} W_{t}-\frac{1}{2} \sigma^{2}\left(t, x_{0}\right) v_{t} \mathrm{~d} t, \quad X_{0}^{P}=x_{0}, \\
v_{t}=v_{0}+\int_{0}^{t} \alpha_{s} \mathrm{~d} s .
\end{array}\right.
$$

Such an approximation can be justified if the volatility of volatility $\xi$ is small (thus $V_{t} \approx v_{t}$ ) and if one of the two following situations holds, both justifying $\sigma\left(t, X_{t}\right) \approx \sigma\left(t, x_{0}\right)$ : i) the local volatility function $\sigma(t, \cdot)$ has small variations; ii) the local part of the diffusion component is small (i.e. $|\sigma|_{\infty}$ small), which implies $X_{t} \approx x_{0}$. Besides we expect to have even more accurate approximations for small maturities (leading to $X_{t} \approx x_{0}$ and $V_{t} \approx v_{0}, t \in[0, T]$ ). These features are encoded in the error bounds of Theorems 2.4, 4.2 and 4.4, that read as multi-parameter error estimates. The above assumptions are rather realistic in practice, or nonetheless they define a domain of validity of our approximations.

In spite of the inspiration by [BGM10b], the mathematical analysis of error estimates must be quite different since the model (1.2)-(1.3) is not smooth enough in the Malliavin sense (because of the CIR process, see [AE08, De 11]). We briefly explain how we overcome this major problem, the 
arguments are interesting on their own and could be transposed to other studies: we use Malliavin calculus for a suitably perturbed payoff $h_{\delta}$ and a suitably perturbed random variable $\bar{X}_{T}$, this is inspired by [Fou08] but in a different context. Let us consider the Gaussian regularization $h_{\delta}(x):=$ $\mathbb{E}\left[h\left(x+\delta \bar{W}_{T}\right)\right]$ for an independent Brownian motion $\bar{W}$ and a small parameter $\delta>0$ (fixed later); we use the splitting-noise property $h_{\delta}(x)=\mathbb{E}\left[h_{\delta / \sqrt{2}}\left(x+\delta \bar{W}_{\frac{T}{2}}\right)\right]$. Write the decomposition

$$
\mathbb{E}\left[h\left(X_{T}\right)\right] \approx \mathbb{E}\left[h_{\delta}\left(\bar{X}_{T}\right)\right] \approx \mathbb{E}\left[h_{\delta}\left(X_{T}^{P}\right)\right]+\mathbb{E}\left[h_{\delta}^{\prime}\left(X_{T}^{P}\right)\left(\bar{X}_{T}-X_{T}^{P}\right)\right]+\ldots
$$

i) Since $h$ is Lipschitz, the first approximation is easily justified if $\delta$ is small enough and if $X_{T}$ and $\bar{X}_{T}$ are close enough in $L^{p}$-sense.

ii) The last expectation is computed (up to an error) using the techniques of [BGM09, BGM10b], and although cumbersome, the computations may be considered now as standard and yield the existence of explicit coefficients $\left(C_{k}\right)_{k}$ such that

$$
\begin{aligned}
\mathbb{E}\left[h_{\delta}^{\prime}\left(X_{T}^{P}\right)\left(\bar{X}_{T}-X_{T}^{P}\right)\right] & =\left.\sum_{k} C_{k} \partial_{x^{k}}^{k} \mathbb{E}\left[h_{\delta}\left(X_{T}^{P}+x\right)\right]\right|_{x=0}+\text { error } \\
& =\left.\sum_{k} C_{k} \partial_{x^{k}}^{k} \mathbb{E}\left[h\left(X_{T}^{P}+x\right)\right]\right|_{x=0}+\text { other error } .
\end{aligned}
$$

iii) The tough part of the analysis is related to the global error control, which enlightens the right choice of $\delta$ and $\bar{X}_{T}$. To account for non-smooth payoffs we use an integration by parts formula, which relies on the non-degeneracy of the interpolated random variable $X^{\lambda}:=$ $\lambda \bar{X}_{T}+(1-\lambda) X_{T}^{P}$ (for any fixed $\left.\lambda \in\right] 0,1\left[\right.$ ). Note that this excludes to take $\bar{X}_{T}=X_{T}$ that is not sufficiently Malliavin differentiable. Alternatively, we select a $\bar{X}_{T} \in \mathbb{D}^{\infty}$ which on the one hand, is close enough to $X_{T}$ in $L^{p}$, and which, on the other hand, is such that $X^{\lambda}$ is nondegenerate with high probability under the sole assumption $\int_{0}^{T} \sigma\left(t, x_{0}\right)^{2} v_{t} \mathrm{~d} t>0$ (which we call $\left(\mathcal{H}_{x_{0}}\right)$ and which reads as a pointwise ellipticity assumption). The non-degeneracy of $X^{\lambda}$ on a subset of $\Omega$ is not sufficient for an integration-by-parts formula for $\mathbb{E}\left[\partial_{x}^{k} h_{\delta}\left(X^{\lambda}\right) Y\right]$ ( $Y$ is an arbitrary random variable): but due to the splitting-noise property, the above expectation is equal to $\mathbb{E}\left[\partial_{x}^{k} h_{\delta / \sqrt{2}}\left(X^{\lambda}+\delta \bar{W}_{\frac{T}{2}}\right) Y\right]$, moreover $X^{\lambda}+\delta \bar{W}_{\frac{T}{2}}$ is uniformly non-degenerate, with nice estimates provided that $\delta$ is not too small.

The precise tuning of $\delta$ and the construction of $\bar{X}_{T}$ are detailed in Section 3. The final approximation formula (Theorem 2.4) takes the form of an explicit Gaussian representation

$$
\mathbb{E}\left[h\left(X_{T}\right)\right]=\mathbb{E}\left[h\left(X_{T}^{P}\right)\right]+\left.\sum_{k} C_{k} \partial_{x^{k}}^{k} \mathbb{E}\left[h\left(X_{T}^{P}+x\right)\right]\right|_{x=0}+\text { error. }
$$

where the above derivatives write as Greeks within the Gaussian model.

To summarize, the main strengths of our work are fourfold: (1) explicit expansions for bounded Lipschitz payoffs and for the implied volatility, (2) allowing time-dependent parameters and assetvolatility correlation, (3) non-asymptotic error analysis (dependence of error with respect to multiparameters) under a local non-degeneracy condition $\left(\mathcal{H}_{x_{0}}\right),(4)$ rigorous error analysis taking into account that $V$ has a non-smooth coefficient.

$\triangleright$ Comparison with the literature. In the two last decades, numerous papers have been devoted to the analytical approximation of financial models. For an overview, see [BG12, Gul12, FGG $\left.{ }^{+} 15\right]$ among others. Here, we mention the works that are the most related to our setting, and we emphasize the main differences compared to our works (keeping in mind the above main features). 
Firstly, some authors focus on small maturity asymptotics and use geodesic tools to compute the main expansion terms: see [BBF04, Hen08, Lew07] where short maturity implied volatility approximations are obtained. First expansion terms are derived for general local and stochastic volatilities models in [FJ11] but under the assumptions of null correlation and under rather strong hypotheses of the volatility coefficients (excluding the CIR process). The asymptotic expansion of the density function in the SABR model is investigated in [JT11]. Similar results are derived using PDE (parametrix, adjoint expansion) and Fourier arguments by Pascucci etal. [PP13] for general time-independent local-Heston volatility models, however the error analysis is not handled; extensions to general stochastic volatility models are performed in [LPP14] under stronger smoothness assumptions (excluding the CIR process) and uniform ellipticity conditions (stronger than in our setting, where we assume only that the initial volatility is not 0 and the correlation is arbitrary in $[-1,1])$. Observe that our non-degeneracy pointwise condition $\left(\mathcal{H}_{x_{0}}\right)$ does not imply that the law of $X_{T}$ has a density or that related PDEs have solution in the classical sense, which illustrates the difference with the setting of [LPP14].

Secondly, regarding long maturity asymptotics, Forde and Pogudin in [FP13] study the cases of SABR and CEV-Heston models in different strike regimes, but mainly assuming null-correlation.

Thirdly, the homogenization/multiscales approach by Fouque etal. [FPSK11] is developed in [FL11] to perform an asymptotic expansion w.r.t. a fast mean reversion parameter of the CIR volatility, in a model of type (1.2)-(1.3) with time-independent parameters. So far, the aforementioned works (except [LPP14]) assume time-independent parameters.

Fourthly, following the asymptotic expansion method of Watanabe, Takahashi and co-authors provide in a series of works small noise approximations of financial models (see [TY12] about general stochastic volatility models): however, their results can not apply to the current localHeston volatility model since it is not smooth in Malliavin sense.

$\triangleright$ Outline of the paper. The paper is organized as follows. In Section 2 we state a third order price approximation formula with error bounds (Theorem 2.4), which is the main result. Section 3 is devoted to the proof, which constitutes the technical core of the paper, interesting on its own. For the sake of clarity, we first give an outline of the proof, in a rather heuristic way, to highlight the main steps, the major difficulties and to sketch arguments to overcome them. The explicit calculus of the expansion coefficients is postponed to Appendix B. In Section 4 we apply our expansion formula to the particular case of Call/Put payoffs to derive implied volatility expansions with local volatility frozen at spot and at mid-point (between strike and spot). Results are stated in Theorems 4.2 and 4.4. Section 5 gathers numerical experiments illustrating the approximation formula accuracy, taking as a benchmark the Monte Carlo method. In Appendix, we give intermediate and complementary results.

\section{Main Result.}

\subsection{Notations and definitions.}

$\triangleright$ Extremes of deterministic functions. For measurable and bounded functions $f:[0, T] \rightarrow \mathbb{R}$ and $g:[0, T] \times \mathbb{R} \rightarrow \mathbb{R}$, we define $f_{\text {inf }}:=\operatorname{ess} \inf _{t \in[0, T]} f_{t}, f_{\text {sup }}:=\operatorname{ess} \sup _{t \in[0, T]} f_{t},|g|_{\infty}:=\operatorname{ess} \sup _{t \in[0, T], x \in \mathbb{R}}|g(t, x)|$. $\triangleright$ Differentiation and integration. We denote by $\mathcal{C}_{b}^{\infty}(\mathbb{R})$, the space of real-valued infinitely differentiable functions, bounded with bounded derivatives. For a sufficiently smooth function $\psi$ : $[0, T] \times \mathbb{R} \mapsto \mathbb{R}$, we write: $\psi_{t}^{(i)}(x)=\partial_{x^{i}}^{i} \psi(t, x)$. When considering the spatial point $x_{0}$, we often 
use the notations $\psi_{t}=\psi_{t}\left(x_{0}\right)$ and $\psi_{t}^{(i)}=\psi_{t}^{(i)}\left(x_{0}\right)$ whenever unambiguous.

Definition 2.1. The integral operator $\omega^{T}$ and its n-times iteration are defined as follows: for any integrable functions $l, l_{1}, \cdots, l_{n}$ and any $t \in[0, T]$,

$$
\omega(l)_{t}^{T}:=\int_{t}^{T} l_{u} d u \quad \text { and } \quad \omega\left(l_{1}, \cdots, l_{n}\right)_{t}^{T}:=\omega\left(l_{1} \omega\left(l_{2}, \cdots, l_{n}\right)^{T}\right)_{t}^{T} .
$$

DEFINITION 2.2. For $h: \mathbb{R} \mapsto \mathbb{R}$ a payoff function with exponential growth, the $i$-th Greek for $X_{T}^{P}$ and $h$ is defined ${ }^{1}$ by

$$
\mathcal{G}_{i}^{h}:=\left.\partial_{x^{i}}^{i} \mathbb{E}\left[h\left(X_{T}^{P}+x\right)\right]\right|_{x=0} .
$$

Given appropriate smoothness assumptions on $h$, one has $\mathcal{G}_{i}^{h}=\mathbb{E}\left[h^{(i)}\left(X_{T}^{P}\right)\right]$.

$\triangleright$ Assumptions on $\sigma($.$) and \left(V_{t}\right)_{t \leq T}$.

$\left(\mathcal{H}_{x_{0}}\right): \sigma($.$) is a bounded measurable function of (t, x) \in[0, T] \times \mathbb{R}$ and three times continuously differentiable w.r.t. $x$ with bounded derivatives. Set

$$
\mathcal{M}_{1}(\sigma)=\max _{1 \leq i \leq 3}\left|\partial_{x^{i}}^{i} \sigma(.)\right|_{\infty} \text { and } \mathcal{M}_{0}(\sigma)=\max _{0 \leq i \leq 3}\left|\partial_{x^{i}}^{i} \sigma(.)\right|_{\infty} .
$$

In addition, we assume the pointwise ellipticity condition $\int_{0}^{T} \sigma_{t}^{2} v_{t} \mathrm{~d} t>0$ where we recall $\sigma_{t}=\sigma\left(t, x_{0}\right)$.

$(\mathcal{P}): \alpha$ and $\xi$ are measurable, bounded on $[0, T]$ and positive, with $\xi_{\text {inf }}>0$ and $2\left(\frac{\alpha}{\xi^{2}}\right)_{\text {inf }} \geq 1$.

Because there exists a unique process $\left(V_{t}\right)_{t \leq T}$ satisfying the $\operatorname{SDE}(1.3),\left(\mathcal{H}_{x_{0}}\right)$ guarantees the existence and the uniqueness of a solution for (1.2), considering generalized stochastic integration w.r.t. semi-martingales (see [Pro04, Theorem 6 p. 249]). Remind (see [BGM10b, Lemma 4.2]) that $(\mathcal{P})$ implies that $\mathbb{P}\left(\forall t \in[0, T]: V_{t}>0\right)=1$. Additionally, the asset price process is a martingale, which is proved in Appendix A.

Proposition 2.3. Under $\left(\mathcal{H}_{x_{0}}\right)$ and $(\mathcal{P}),\left(e^{X_{t}}\right)_{0 \leq t \leq T}$ is a martingale.

This property will be used in Subsection 2.3 and Section 4 .

$\triangleright$ Assumptions on the payoff function. For realistic interesting applications in finance, we consider the space $\operatorname{Lip}_{\mathrm{b}}(\mathbb{R})$ of Lipschitz bounded functions $h$, i.e. satisfying

$$
C_{h}:=\sup _{x \in \mathbb{R}}|h(x)|<+\infty, \quad L_{h}:=\sup _{x \neq y,(x, y) \in \mathbb{R}^{2}}\left|\frac{h(y)-h(x)}{y-x}\right|<+\infty .
$$

This space includes the classical Put payoff function $x \rightarrow\left(K-e^{x}\right)_{+}$with strike $K$. Relaxing the boundedness assumption may lead to ill-posedness problems since the model (1.2)-(1.3) may be such that $\mathbb{E}\left[\left(e^{X_{T}}\right)^{p}\right]=+\infty$ for some $p>1$ (see [AP07]). Nevertheless, our approximation formula extends to the Call payoff owing to the Call/Put relation (valid in view of Proposition $2.3): \mathbb{E}\left[\left(e^{X_{T}}-K\right)_{+}\right]=e^{x_{0}}-K+\mathbb{E}\left[\left(K-e^{X_{T}}\right)_{+}\right]$.

$\triangleright$ Generic constants and upper bounds. We keep the same notation $c$ for all non-negative constants depending on: universal constants, on a number $p \geq 1$ arising in $L^{p}$ estimates, in a non decreasing way on $\xi_{\text {sup }}, \mathcal{M}_{0}(\sigma), \mathcal{M}_{1}(\sigma), T, \frac{|\sigma|_{\infty}^{2} T}{\int_{0}^{T} \sigma_{t}^{2} v_{t} \mathrm{~d} t}, v_{0}, 1 / v_{0}$ and $\alpha_{\text {sup }}$. We frequently use the short notation $A \leq_{c} B$ for positive $A$ which means that $A \leq c B$ for a generic constant $c$. Similarly " $A=\mathcal{O}(B)$ " means that $|A| \leq c B$ for a generic constant $c$.

$\triangleright$ Miscellaneous. The $L^{p}$-norm $(p \geq 1)$ of a random variable is denoted by $\|.\|_{p}$.

\footnotetext{
${ }^{1}$ well-defined, even if $h$ is not smooth, as soon as the variance $\int_{0}^{T} \sigma_{t}^{2} v_{t} \mathrm{~d} t$ of the Gaussian random variable $X_{T}^{P}$ is positive, i.e. our assumption $\left(\mathcal{H}_{x_{0}}\right)$.
} 
2.2. Third order approximation price formula. We state the main result of the paper:

THEOREM 2.4 (3rd order approximation price formula). Assume $\left(\mathcal{H}_{x_{0}}\right)$ and $(\mathcal{P})$. Then for any $h \in \operatorname{Lip}_{\mathrm{b}}(\mathbb{R})$, we have:

$$
\mathbb{E}\left[h\left(X_{T}\right)\right]=\mathbb{E}\left[h\left(X_{T}^{P}\right)\right]+\sum_{i=1}^{6} \eta_{i, T} \mathcal{G}_{i}^{h}+\text { Error }_{3, h},
$$

where:

$$
\begin{aligned}
\eta_{1, T}:= & \frac{C_{1, T}^{l}}{2}-\frac{C_{2, T}^{l}}{2}-\frac{C_{3, T}^{l}}{4}-\frac{C_{4, T}^{l}}{2}-C_{1, T}^{l s}, \\
\eta_{2, T}:= & -\frac{3 C_{1, T}^{l}}{2}+\frac{C_{2, T}^{l}}{2}+\frac{5 C_{3, T}^{l}}{4}+\frac{7 C_{4, T}^{l}}{2}+\frac{\left(C_{1, T}^{l}\right)^{2}}{8}-\frac{C_{1, T}^{s}}{2} \\
& +\frac{C_{3, T}^{s}}{4}+C_{1, T}^{l s}+\frac{C_{2, T}^{l s}}{2}+\frac{C_{3, T}^{l s}}{2}+C_{4, T}^{l s}+\frac{C_{5, T}^{l s}}{2}+\frac{C_{6, T}^{l s}}{4}, \\
\eta_{3, T}:= & C_{1, T}^{l}-2 C_{3, T}^{l}-6 C_{4, T}^{l}-\frac{3\left(C_{1, T}^{l}\right)^{2}}{4}+\frac{C_{1, T}^{s}}{2}-\frac{C_{2, T}^{s}}{2}-\frac{C_{3, T}^{s}}{2} \\
& -\frac{3 C_{2, T}^{l s}}{2}-\frac{3 C_{3, T}^{l s}}{2}-\frac{5 C_{4, T}^{l s}}{2}-C_{5, T}^{l s}-\frac{3 C_{6, T}^{l s}}{4}-\frac{C_{1, T}^{l} C_{1, T}^{s}}{4}, \\
\eta_{4, T}:= & C_{3, T}^{l}+3 C_{4, T}^{l}+\frac{13\left(C_{1, T}^{l}\right)^{2}}{8}+\frac{C_{2, T}^{s}}{2}+\frac{C_{3, T}^{s}}{4}+C_{2, T}^{l s} \\
& +C_{3, T}^{l s}+\frac{3 C_{4, T}^{l s}}{2}+\frac{C_{5, T}^{l s}}{2}+\frac{C_{6, T}^{l s}}{2}+\frac{\left(C_{1, T}^{s}\right)^{2}}{8}+C_{1, T}^{l} C_{1, T}^{s}, \\
\eta_{5, T}:= & -\frac{3\left(C_{1, T}^{l}\right)^{2}}{2}-\frac{\left(C_{1, T}^{s}\right)^{2}}{4}-\frac{5 C_{1, T}^{l} C_{1, T}^{s}}{4}, \\
\eta_{6, T}:= & \frac{\left(C_{1, T}^{l}\right)^{2}}{2}+\frac{\left(C_{1, T}^{s}\right)^{2}}{8}+\frac{C_{1, T}^{l} C_{1, T}^{s}}{2},
\end{aligned}
$$

and:

$$
\begin{array}{ll}
C_{1, T}^{l}:=\omega\left(\sigma^{2} v, \sigma \sigma^{(1)} v\right)_{0}^{T}, & C_{2, T}^{l}:=\omega\left(\sigma^{2} v,\left(\left(\sigma^{(1)}\right)^{2}+\sigma \sigma^{(2)}\right) v\right)_{0}^{T}, \\
C_{3, T}^{l}:=\omega\left(\sigma^{2} v, \sigma^{2} v,\left(\left(\sigma^{(1)}\right)^{2}+\sigma \sigma^{(2)}\right) v\right)_{0}^{T}, & C_{4, T}^{l}:=\omega\left(\sigma^{2} v, \sigma \sigma^{(1)} v, \sigma \sigma^{(1)} v\right)_{0}^{T}, \\
C_{1, T}^{s}:=\omega\left(\rho \xi \sigma v, \sigma^{2}\right)_{0}^{T}, & C_{2, T}^{s}:=\omega\left(\rho \xi \sigma v, \rho \xi \sigma, \sigma^{2}\right)_{0}^{T}, \\
C_{3, T}^{s}:=\omega\left(\xi^{2} v, \sigma^{2}, \sigma^{2}\right)_{0}^{T}, & \\
C_{1, T}^{l s}:=\omega\left(\rho \xi \sigma v, \sigma \sigma^{(1)}\right)_{0}^{T}, & C_{2, T}^{l s}:=\omega\left(\rho \xi \sigma v, \sigma^{2} v, \sigma \sigma^{(1)}\right)_{0}^{T}, \\
C_{3, T}^{l s}:=\omega\left(\sigma^{2} v, \rho \xi \sigma v, \sigma \sigma^{(1)}\right)_{0}^{T}, & C_{4, T}^{l s}:=\omega\left(\rho \xi \sigma v, \sigma^{2}, \sigma \sigma^{(1)} v\right)_{0}^{T}, \\
C_{5, T}^{l s}:=\omega\left(\rho \xi \sigma v, \sigma \sigma^{(1)} v, \sigma^{2}\right)_{0}^{T}, & C_{6, T}^{l s}:=\omega\left(\sigma^{2} v, \rho \xi \sigma^{(1)} v, \sigma^{2}\right)_{0}^{T} .
\end{array}
$$

Then the approximation error is estimated as follows:

$$
\text { Error }_{3, h}=\mathcal{O}\left(L_{h}|\sigma|_{\infty}\left[\xi_{\text {sup }}^{3}+\mathcal{M}_{1}(\sigma)\left(\mathcal{M}_{0}(\sigma)+\xi_{\text {sup }}\right)^{2}\right] T^{2}\right) .
$$

The notations $C^{l}, C^{s}, C^{l s}$ refer to the dependency of the coefficients w.r.t. the local volatility only, to the stochastic one, or to both (see explanations in Subsection 2.3).

We make several additional remarks. Firstly, note that contrary to [BGM10b, Theorem 2.2], we do not assume anymore that the correlation is bounded away from -1 and 1 . This is a nice improvement. 
Secondly, the error bound (2.4) justifies the label of third order approximation formula because using the notation $M=\max \left(\mathcal{M}_{0}(\sigma), \xi_{\text {sup }}\right)$, we readily have Error ${ }_{3, \mathrm{~h}}=\mathcal{O}\left((M \sqrt{T})^{4}\right)$. Besides, making reference to the introduction, we retrieve that if $|\sigma|_{\infty}=0$ or $\max \left(\mathcal{M}_{1}(\sigma), \xi_{\text {sup }}\right)=0$ or $T=0$, the approximation formula (2.3) is exact (the model and the proxy coincide and the $C$ coefficients vanish). Obviously if $L_{h}=0$ (i.e. constant $h$ ), the error and sensitivities are equal to zero.

Thirdly, if one prefers to restrict to a second order approximation, it simply writes:

$$
\begin{aligned}
\mathbb{E}\left[h\left(X_{T}\right)\right]= & \mathbb{E}\left[h\left(X_{T}^{P}\right)\right]+C_{1, T}^{l}\left[\frac{1}{2} \mathcal{G}_{1}^{h}-\frac{3}{2} \mathcal{G}_{2}^{h}+\mathcal{G}_{3}^{h}\right]+C_{1, T}^{s}\left[-\frac{\mathcal{G}_{2}^{h}}{2}+\frac{\mathcal{G}_{3}^{h}}{2}\right] \\
& +\mathcal{O}\left(L_{h}|\sigma|_{\infty}\left[\xi_{\text {sup }}^{2}+\mathcal{M}_{1}(\sigma)\left(\mathcal{M}_{0}(\sigma)+\xi_{\text {sup }}\right)\right] T^{\frac{3}{2}}\right) .
\end{aligned}
$$

We let the reader verify that the additional corrective terms of the expansion (2.3) are bounded (up to generic constants) by $L_{h}|\sigma|_{\infty}\left[\xi_{\text {sup }}^{2}+\mathcal{M}_{1}(\sigma)\left(\mathcal{M}_{0}(\sigma)+\xi_{\text {sup }}\right)\right] T^{\frac{3}{2}}$ using standard upper bounds for the derivatives of the Gaussian density and for the neglected coefficients $C_{{ }^{*} T}$.

\subsection{Corollaries.}

$\triangleright$ Particular cases of pure local volatility model and pure Heston model.

a) When $\xi_{\text {sup }}$ is equal to zero, the coefficients $C^{s}$ and $C^{l s}$ are null: then we exactly retrieve the expansion of the pure local volatility model proposed in [BGM10a]. The terms $C^{l}$ therefore read as purely local volatility contributions.

b) If $\mathcal{M}_{1}(\sigma)=0$ (case of pure Heston model), all the coefficients $C^{l}$ and $C^{l s}$ are equal to zero: we can retrieve the expansion derived in [BGM10b], by taking into account our parametrization of $V$ and by transforming the sensitivities w.r.t. the total variance in [BGM10b, Theorem 2.2] into sensitivities w.r.t. the log-spot.

c) Finally we interpret the coefficients $C^{l s}$ as a contribution related to the mixture of both the local and stochastic parts of the volatility. All these terms notably depend on the correlation. In case of independent $W$ and $B$, all the coefficients are equal to 0 except the $C^{l}$ terms and $C_{3, T}^{s}$.

$\triangleright$ Applications to Call payoff function. One can directly apply this theorem for the Put payoff function $h(x)=\left(K-e^{x}\right)_{+}$. The reader should remark that the above expansion formula is exact for the particular payoff function $h(x)=\exp (x)$ : indeed $\mathbb{E}\left[h\left(X_{T}\right)\right]=\mathbb{E}\left[h\left(X_{T}^{P}\right)\right]=\mathcal{G}_{i}^{\exp (.)}=e^{x_{0}}$ and the sum of the corrective terms is equal to zero, $\sum_{i=1}^{6} \eta_{i, T} \mathcal{G}_{i}^{\exp (.)}=e^{x_{0}} \sum_{i=1}^{6} \eta_{i, T}=0$. Therefore the expansion remains valid for the Call payoff function $h(x)=\left(e^{x}-K\right)_{+}$although $h \notin \operatorname{Lip}_{\mathrm{b}}(\mathbb{R})$, and furthermore, the Call/Put parity relationship (valid owing to Proposition 2.3) is preserved within these approximations.

\section{Error analysis.}

3.1. Outline of the proof. To relate the initial process (1.2)-(1.3) to the proxy process (1.4), we introduce a two-dimensional parameterized process given by:

$$
\begin{aligned}
\mathrm{d} X_{t}^{\eta} & =\sigma\left(t, \eta X_{t}^{\eta}+(1-\eta) x_{0}\right) \sqrt{V_{t}^{\eta}} \mathrm{d} W_{t}-\frac{1}{2} \sigma^{2}\left(t, \eta X_{t}^{\eta}+(1-\eta) x_{0}\right) V_{t}^{\eta} \mathrm{d} t, \quad X_{0}^{\eta}=x_{0}, \\
\mathrm{~d} V_{t}^{\eta} & =\alpha_{t} \mathrm{~d} t+\eta \xi_{t} \sqrt{V_{t}^{\eta}} \mathrm{d} B_{t}, V_{0}^{\eta}=v_{0},
\end{aligned}
$$

where $\eta$ is an interpolation parameter lying in the range $[0,1]$, so that on the one hand for $\eta=1$, $X_{t}^{1}=X_{t}$ and $V_{t}^{1}=V_{t}$, and on the other hand for $\eta=0, X_{t}^{0}=X_{t}^{P}$ and $V_{t}^{0}=v_{t}$. This parameterization is a tricky interpolation between models, which goal is to derive successive corrective 
processes in order to obtain a tractable explicit approximation formula. In addition $(\mathcal{P})$ implies that $\forall \eta \in[0,1], \mathbb{P}\left(\forall t \in[0, T]: V_{t}^{\eta}>0\right)=1$ (see [BGM10b, Lemma 4.2]).

We define the stochastic volatility process:

Definition 3.1. $\Lambda_{t}^{\eta}=\sqrt{V_{t}^{\eta}}, \forall t \in[0, T], \forall \eta \in[0,1]$.

In addition, we introduce $\left(\lambda_{t}\right)_{t \in[0, T]}$ defined for any $t \in[0, T]$ by:

$$
\lambda_{t}=\Lambda_{t}^{\eta=0}=\sqrt{V_{t}^{0}}=\sqrt{v_{t}}=\sqrt{v_{0}+\int_{0}^{t} \alpha_{s} \mathrm{~d} s} .
$$

We present here a sketch of proof in order to fix the main ideas and to highlight the main difficulties. The strategy of approximation has been globally explained in introduction.

$\triangleright$ 1st step. We construct corrective processes to approximate $X_{T}$ in $L^{p}$. Consider the parameterized process defined in (3.1)-(3.2). We recall that the Gaussian proxy process $\left(X_{t}^{P}\right)_{t \in[0, T]}$ defined in (1.4) is obtained by setting $\eta=0$. The following corrective processes $\left(X_{i, t}\right)_{t \in[0, T]^{-}}\left(V_{i, t}\right)_{t \in[0, T]^{-}}$ $\left(\Lambda_{i, t}\right)_{t \in[0, T]}$ for $i \in\{1,2\}$ are obtained by a formal $i$-times differentiation of (3.1)-(3.2) w.r.t. $\eta$ and by taking $\eta=0$ thereafter. For the first corrective processes, we obtain:

$$
\begin{aligned}
\mathrm{d} X_{1, t} & =\left[\left(X_{t}^{P}-x_{0}\right) \sigma_{t}^{(1)} \lambda_{t}+\Lambda_{1, t} \sigma_{t}\right]\left(\mathrm{d} W_{t}-\sigma_{t} \lambda_{t} \mathrm{~d} t\right), \quad X_{1,0}=0, \\
V_{1, t} & =\int_{0}^{t} \xi_{s} \lambda_{s} \mathrm{~d} B_{s} \\
\Lambda_{1, t} & =\frac{V_{1, t}}{2 \lambda_{t}}
\end{aligned}
$$

The second corrective processes are:

$$
\begin{aligned}
\mathrm{d} X_{2, t}= & \left\{\lambda_{t}\left[\left(X_{t}^{P}-x_{0}\right)^{2} \sigma_{t}^{(2)}+2 X_{1, t} \sigma_{t}^{(1)}\right]+2\left(X_{t}^{P}-x_{0}\right) \Lambda_{1, t} \sigma_{t}^{(1)}\right\}\left(\mathrm{d} W_{t}-\sigma_{t} \lambda_{t} \mathrm{~d} t\right) \\
& +\left\{\Lambda_{2, t} \sigma_{t} \mathrm{~d} W_{t}-\left[\left(X_{t}^{P}-x_{0}\right) V_{1, t} \sigma_{t}^{(1)} \sigma_{t}+\left(X_{t}^{P}-x_{0}\right)^{2}\left(\sigma_{t}^{(1)}\right)^{2} v_{t}+\frac{V_{2, t}}{2} \sigma_{t}^{2}\right] \mathrm{d} t\right\}, X_{2,0}=0 \\
V_{2, t}= & \int_{0}^{t} \xi_{s} \frac{V_{1, s}}{\lambda_{s}} \mathrm{~d} B_{s} \\
\Lambda_{2, t}= & \frac{V_{2, t}}{2 \lambda_{t}}-\frac{V_{1, t}^{2}}{4\left(\lambda_{t}\right)^{3}}
\end{aligned}
$$

Under $\left(\mathcal{H}_{x_{0}}\right)$, these corrective processes $\left(X_{i, t}\right)_{t \in[0, T]^{-}}\left(V_{i, t}\right)_{t \in[0, T]^{-}}\left(\Lambda_{i, t}\right)_{t \in[0, T]}$ for $i \in\{1,2\}$ are well defined.

$\triangleright$ 2nd step. We compute the corrective terms. To start with, assume that $h \in \mathcal{C}_{b}^{\infty}(\mathbb{R})$ and perform a third order Taylor expansion for the function $h$ at $x=X_{T}$ around $x=X_{T}^{P}$ :

$$
\begin{aligned}
\mathbb{E}\left[h\left(X_{T}\right)\right]= & \mathbb{E}\left[h\left(X_{T}^{P}\right)\right]+\mathbb{E}\left[h^{(1)}\left(X_{T}^{P}\right)\left(X_{T}-X_{T}^{P}\right)\right]+\frac{1}{2} \mathbb{E}\left[h^{(2)}\left(X_{T}^{P}\right)\left(X_{T}-X_{T}^{P}\right)^{2}\right] \\
& +\mathbb{E}\left[\left(X_{T}-X_{T}^{P}\right)^{3} \int_{0}^{1} h^{(3)}\left(X_{T}^{P}+\eta\left(X_{T}-X_{T}^{P}\right) \frac{(1-\eta)^{2}}{2} \mathrm{~d} \eta\right]\right. \\
= & \mathbb{E}\left[h\left(X_{T}^{P}\right)\right]+\mathbb{E}\left[h^{(1)}\left(X_{T}^{P}\right) X_{1, T}\right]+\mathbb{E}\left[h^{(1)}\left(X_{T}^{P}\right) \frac{X_{2, T}}{2}\right]+\frac{1}{2} \mathbb{E}\left[h^{(2)}\left(X_{T}^{P}\right) X_{1, T}^{2}\right]+\text { Error }_{3, \mathrm{~h}}, \\
\text { Error }_{3, h}= & \mathbb{E}\left[h^{(1)}\left(X_{T}^{P}\right)\left(X_{T}-\sum_{j=0}^{2} \frac{X_{j, T}}{j !}\right)\right]+\frac{1}{2} \mathbb{E}\left[h^{(2)}\left(X_{T}^{P}\right)\left(X_{T}-X_{T}^{P}-X_{1, T}\right)\left(X_{T}-X_{T}^{P}+X_{1, T}\right)\right]
\end{aligned}
$$




$$
+\mathbb{E}\left[\left(X_{T}-X_{T}^{P}\right)^{3} \int_{0}^{1} h^{(3)}\left(X_{T}^{P}+\eta\left(X_{T}-X_{T}^{P}\right)\right) \frac{(1-\eta)^{2}}{2} \mathrm{~d} \eta\right]
$$

with the convention $X_{T}^{P}=X_{T}^{0}=X_{0, T}$. Then we transform the terms $\mathbb{E}\left[h^{(1)}\left(X_{T}^{P}\right) X_{1, T}\right], \mathbb{E}\left[h^{(1)}\left(X_{T}^{P}\right) \frac{X_{2, T}}{2}\right]$ and $\frac{1}{2} \mathbb{E}\left[h^{(2)}\left(X_{T}^{P}\right) X_{1, T}^{2}\right]$ into a weighted sum of sensitivities, yielding the sum in (2.3). To achieve this transformation, we apply a key lemma which proof is postponed to Appendix B:

Lemma 3.2. Let $\varphi$ be a $\mathcal{C}_{b}^{\infty}(\mathbb{R})$ function and $\left(f_{t}\right)_{t}$ be a measurable and bounded deterministic function. Let $N \geq 1$ be fixed, and consider measurable and bounded deterministic functions $t \mapsto l_{i, t}$ for $i=1, \ldots, N$. Then, using the convention $\mathrm{d} W_{t}^{0}=\mathrm{d} t, \mathrm{~d} W_{t}^{1}=\mathrm{d} W_{t}$ and $\mathrm{d} W_{t}^{2}=\mathrm{d} B_{t}$, for any $\left(I_{1}, \ldots, I_{N}\right) \in\{0,1,2\}^{N}$ we have:

$$
\begin{aligned}
& \mathbb{E}\left[\varphi\left(\int_{0}^{T} f_{t} \mathrm{~d} W_{t}\right) \int_{0}^{T} l_{N, t_{N}} \int_{0}^{t_{N}} l_{N-1, t_{N}-1} \ldots \int_{0}^{t_{2}} l_{1, t_{1}} \mathrm{~d} W_{t_{1}}^{I_{1}} \ldots \mathrm{d} W_{t_{N-1}}^{I_{N-1}} \mathrm{~d} W_{t_{N}}^{I_{N}}\right] \\
& =\left.\omega\left(\widehat{l}_{1}, \ldots, \widehat{l}_{N}\right)_{0}^{T} \partial_{x \neq\left\{k: I_{k} \neq 0\right\}}^{\#\left\{k: I_{k} \neq 0\right\}} \mathbb{E}\left[\varphi\left(\int_{0}^{T} f_{t} \mathrm{~d} W_{t}+x\right)\right]\right|_{x=0},
\end{aligned}
$$

where $\widehat{l}_{k, t}:= \begin{cases}l_{k, t} & \text { if } I_{k}=0, \\ f_{t} l_{k, t} & \text { if } I_{k}=1, \\ f_{t} \rho_{t} l_{k, t} & \text { if } I_{k}=2 .\end{cases}$

Details of the complete derivation of the corrective terms appearing in (2.3) is given in Appendix B. Remind that these sensitivities are well defined even if $h$ is not smooth.

$\triangleright$ 3rd step: error analysis. Last but not least, one has to estimate the residual terms. In the smooth case for $h$, owing to (3.10), it is sufficient to estimate the $L^{p}$ norms of the residual processes $X_{T}-\sum_{j=0}^{i} \frac{X_{j, T}}{j !}$ for $i \in\{1,2\}$ and there is no other difficulties in order to conclude. Under the sole assumption that $h \in \operatorname{Lip}_{\mathrm{b}}(\mathbb{R})$, the previous decomposition doesn't apply (because of $h^{(2)}$ and $h^{(3)}$ ). To get rid off the derivatives of $h$, a natural idea is first to smooth $h$, second to employ Malliavin integration by parts formulas, and then to take the smoothing parameter to 0. But this strategy applied to the representation (3.10) fails because the random variable $X_{T}^{P}+\eta\left(X_{T}-X_{T}^{P}\right)$ does not belong to the space $\mathbb{D}^{\infty}$ for $\eta \neq 0$ : indeed, the coefficient function of the square root model does not satisfy the standard assumptions. Malliavin differentiability is studied by hand in [AE08] up to the second order.

To overcome these difficulties, the trick is twofold:

1. we replace $X_{T}$ by the smooth random variable (in Malliavin sense) $X_{T}^{P}+X_{1, T}+\frac{X_{2, T}}{2}$ close to $X_{T}$ in $L^{p}$,

2. we apply a Gaussian regularization to $h$, giving a new payoff $h_{\delta}$ (defined later in (3.32)) which will support the Malliavin calculus computations (inspired by [GM14]).

Therefore $\mathbb{E}\left[h\left(X_{T}\right)\right]$ is approximated by $\mathbb{E}\left[h_{\delta}\left(X_{T}\right)\right]$ which can be decomposed as follows (with similar arguments as (3.12)):

$$
\begin{aligned}
\mathbb{E}\left[h_{\delta}\left(X_{T}\right)\right] & =\mathbb{E}\left[h_{\delta}\left(X_{T}^{P}+X_{1, T}+\frac{X_{2, T}}{2}\right)\right]+\mathbb{E}\left[\left(X_{T}-\sum_{j=0}^{2} \frac{X_{j, T}}{j !}\right) \int_{0}^{1} h_{\delta}^{(1)}\left((1-\eta) \sum_{j=0}^{2} \frac{X_{j, T}}{j !}+\eta X_{T}\right) \mathrm{d} \eta\right] \\
& =\mathbb{E}\left[h_{\delta}\left(X_{T}^{P}\right)\right]+\mathbb{E}\left[h_{\delta}^{(1)}\left(X_{T}^{P}\right)\left(X_{1, T}+\frac{X_{2, T}}{2}\right)\right]+\frac{1}{2} \mathbb{E}\left[h_{\delta}^{(2)}\left(X_{T}^{P}\right) X_{1, T}^{2}\right]+\text { Error }_{3, h_{\delta}},
\end{aligned}
$$




$$
\begin{aligned}
\operatorname{Error}_{3, h_{\delta}}= & \mathbb{E}\left[\left(X_{T}-\sum_{j=0}^{2} \frac{X_{j, T}}{j !}\right) \int_{0}^{1} h_{\delta}^{(1)}\left((1-\eta) \sum_{j=0}^{2} \frac{X_{j, T}}{j !}+\eta X_{T}\right) \mathrm{d} \eta\right] \\
& +\frac{1}{2} \mathbb{E}\left[h_{\delta}^{(2)}\left(X_{T}^{P}\right)\left(X_{1, T} X_{2, T}+\frac{X_{2, T}^{2}}{4}\right)\right] \\
& +\mathbb{E}\left[\left(X_{1, T}+\frac{X_{2, T}}{2}\right)^{3} \int_{0}^{1} \frac{(1-\eta)^{2}}{2} h_{\delta}^{(3)}\left(X_{T}^{P}+\eta\left(X_{1, T}+\frac{X_{2, T}}{2}\right)\right) \mathrm{d} \eta\right] .
\end{aligned}
$$

Observe that the equations (3.11) and (3.14) are the same (up to the modification of $h$ into $h_{\delta}$ ) but the representations of error (3.12) and (3.15) differ. The latter will be that to use in our analysis with Malliavin calculus. As $h \in \operatorname{Lip}_{\mathrm{b}}(\mathbb{R})$, the first term of (3.15) involving only $h_{\delta}^{(1)}$ can be handled directly. Integration by parts from Malliavin calculus should be applied to the two last terms of (3.15), which contain higher derivatives of $h_{\delta}$ with the random variables $X_{T}^{P}, X_{1, T}$ and $X_{2, T}$ belonging to $\mathbb{D}^{\infty}$, but $X_{T}^{P}+\eta\left(X_{1, T}+\frac{X_{2, T}}{2}\right)$ suffers from degeneracy (in the Malliavin sense) for $\eta \neq 0$. This is where Gaussian regularization plays an important role: it allows to integrate by parts w.r.t. the random variable $X_{T}^{P}+\eta\left(X_{1, T}+\frac{X_{2, T}}{2}\right)+$ Gaussian r.v., which is now non degenerate. Details are explained in Subsection 3.3.

The complete analysis of the error estimate (2.4) is given in the following subsection, along several steps:

1. $L^{p}$ norms estimates of the residuals processes,

2. small Gaussian noise perturbation to smooth the function $h$,

3. careful use of Malliavin integration by parts formulas to achieve the proof.

\subsection{Approximation of $X, V, \Lambda$ and error estimates.}

\section{Approximation of $V, \Lambda$ and related error estimates.}

Definition 3.3. Assume $(\mathcal{P})$. We introduce for $i \in\{0,1,2\}$ the $\Lambda$-residual processes defined by $(t \in[0, T])$

$$
R_{i, t}^{\Lambda}:=\Lambda_{t}-\sum_{j=0}^{i} \frac{\Lambda_{j, t}}{j !}
$$

where by convention $\Lambda_{0, t}=\lambda_{t}$ and the corrective processes $\left(\Lambda_{1}, \Lambda_{2}\right)$ are defined in (3.6)-(3.9). By replacing $\Lambda$ by $V$, we define similarly the $V$-residual processes using the notation $R^{V}$.

Proposition 3.4. Assume $(\mathcal{P})$. Then for any $p \geq 1$, we have:

$$
\begin{aligned}
\sqrt{v_{0}} \leq \lambda_{\text {inf }} \leq \lambda_{\text {sup }} & \leq \sqrt{v_{0}+T \alpha_{\text {sup }}}, \\
& \sup _{t \in[0, T]}\left\|\Lambda_{i, t}\right\|_{p} \leq{ }_{c}\left(\xi_{\text {sup }} \sqrt{T}\right)^{i}, \forall i \in\{1,2\}, \\
& \sup _{t \in[0, T]}\left\|R_{i, t}^{\Lambda}\right\|_{p} \leq_{c}\left(\xi_{\text {sup }} \sqrt{T}\right)^{i+1}, \forall i \in\{0,1,2\} .
\end{aligned}
$$

Proof. (3.16) is obvious in view of (3.3). The proofs of (3.17) and (3.18) can be found in [BGM10b, Propositions 4.6, 4.7 and 4.8] replacing in the quoted paper $\kappa$ by 0 and $\kappa \theta_{t}$ by $\alpha_{t}$.

Corollary 3.5. Assume $(\mathcal{P})$. Then for any $p \geq 1$ we have

$$
v_{0} \leq v_{\text {inf }} \leq v_{\text {sup }} \leq v_{0}+T \alpha_{\text {sup }},
$$




$$
\begin{aligned}
& \sup _{t \in[0, T]}\left\|V_{t}\right\|_{p} \leq_{c} 1+v_{0}, \\
& \sup _{t \in[0, T]}\left\|V_{i, t}\right\|_{p} \leq_{c}\left(\xi_{\text {sup }} \sqrt{T}\right)^{i}, \forall i \in\{1,2\}, \\
& \sup _{t \in[0, T]}\left\|R_{i, t}^{V}\right\|_{p} \leq_{c}\left(\xi_{\sup } \sqrt{T}\right)^{i+1}, \forall i \in\{0,1,2\} .
\end{aligned}
$$

Proof. The proofs of (3.19) and (3.20) are easy. The inequality (3.21) is readily obtained thanks to (3.5), (3.8) and (3.17). The proof of (3.22) is available in [BGM10b, Corollary 4.9].

\section{Approximation of $X$ and related error estimates.}

Definition 3.6. Assume $\left(\mathcal{H}_{x_{0}}\right)$. We introduce for $i \in\{0,1,2\}$ the $X$-residual processes defined by $(t \in[0, T])$

$$
R_{i, t}^{X}:=X_{t}-\sum_{j=0}^{i} \frac{X_{j, t}}{j !},
$$

where by convention $X_{0, t}=X_{t}^{0}=X_{t}^{P}$ and the corrective processes $\left(X_{1}, X_{2}\right)$ are defined in (3.4)(3.7). When writing a Taylor expansion of $\sigma_{t}($.$) at x=X_{t}$ around $x=x_{0}$, we denote by $R_{n, \sigma}\left(X_{t}\right)$ the $n^{\text {th }}$ Taylor residual:

$$
R_{n, \sigma}\left(X_{t}\right):=\sigma_{t}\left(X_{t}\right)-\sum_{i=0}^{n} \frac{\left(X_{t}-x_{0}\right)^{i}}{i !} \sigma_{t}^{(i)}
$$

Replacing $\sigma$ by $\sigma^{2}$, we use the similar notation $R_{n, \sigma^{2}}\left(X_{t}\right)$.

Let $p \geq 2$, standard computations involving Burkholder-Davis-Gundy and Holder inequalities yield:

$$
\begin{aligned}
\left\|X_{t}-x_{0}\right\|_{p}^{p} & \leq{ }_{c} t^{\frac{p}{2}-1} \int_{0}^{t}\left\|\sigma_{s}\left(X_{s}\right) \sqrt{V_{s}}\right\|_{p}^{p} \mathrm{~d} s+t^{p-1} \int_{0}^{t}\left\|\sigma_{s}^{2}\left(X_{s}\right) V_{s}\right\|_{p}^{p} \mathrm{~d} s \\
& \leq{ }_{c} t^{\frac{p}{2}-1}|\sigma|_{\infty}^{p} \int_{0}^{t} \mathbb{E}\left[V_{s}^{p / 2}\right] \mathrm{d} s+t^{p-1}|\sigma|_{\infty}^{2 p} \int_{0}^{t} \mathbb{E}\left[V_{s}^{p}\right] \mathrm{d} s \leq_{c}\left(|\sigma|_{\infty} \sqrt{T}\right)^{p},
\end{aligned}
$$

where we have applied the estimate (3.20) at the last inequality. Observe that the above estimate is valid also for $p \in[1,2)$ by using $\|\cdot\|_{p} \leq\|\cdot\|_{q}$ for $p \leq q$.

We now aim at handling $X$-residual processes, and the next results are intermediate steps.

Lemma 3.7. Assume $\left(\mathcal{H}_{x_{0}}\right)$ and $(\mathcal{P})$. For any $p \geq 1$ :

$$
\begin{aligned}
& \sup _{t \in[0, T]}\left\|X_{t}^{P}-x_{0}\right\|_{p} \leq_{c}|\sigma|_{\infty} \sqrt{T}, \\
& \sup _{t \in[0, T]}\left\|\left.X_{i, t}\left|\|_{p} \leq_{c}\right| \sigma\right|_{\infty}\left[\xi_{\text {sup }}^{i}+\mathcal{M}_{1}(\sigma)\left(\mathcal{M}_{0}(\sigma)+\xi_{\text {sup }}\right)^{i-1}\right] T^{\frac{i+1}{2}}, \forall i \in\{1,2\} .\right.
\end{aligned}
$$

Proof. It is enough to prove the inequalities for $p \geq 2$ : thus, now consider such a $p$. The proof of (3.25) is similar to (3.24). For (3.26) $i=1$ : starting from (3.4), the same computations as before give:

$$
\left\|X_{1, t}\right\|_{p} \leq_{c} \mathcal{M}_{1}(\sigma) \sqrt{T}\left(1+\mathcal{M}_{0}(\sigma) \sqrt{T}\right) \sup _{t \in[0, T]}\left\|X_{t}^{P}-x_{0}\right\|_{p}
$$




$$
+|\sigma|_{\infty} \sqrt{T}\left(1+\mathcal{M}_{0}(\sigma) \sqrt{T}\right) \sup _{t \in[0, T]}\left\|V_{1, t}\right\|_{p}
$$

We conclude using (3.25) and (3.21). For (3.26) $i=2$, one has from (3.7):

$$
\begin{aligned}
\left\|X_{2, t}\right\|_{p} \leq{ }_{c} & \mathcal{M}_{1}(\sigma) \sqrt{T}\left(1+\mathcal{M}_{0}(\sigma) \sqrt{T}\right)\left(\sup _{t \in[0, T]}\left\|\left(X_{t}^{P}-x_{0}\right)^{2}\right\|_{p}+\sup _{t \in[0, T]}\left\|X_{1, t}\right\|_{p}\right)+ \\
& +|\sigma|_{\infty} \sqrt{T}\left(1+\mathcal{M}_{0}(\sigma) \sqrt{T}\right) \sup _{t \in[0, T]}\left\|V_{2, t}\right\|_{p}+|\sigma|_{\infty} \sqrt{T} \sup _{t \in[0, T]}\left\|V_{1, t}^{2}\right\|_{p} \\
& +\mathcal{M}_{1}(\sigma) \sqrt{T}\left(1+\mathcal{M}_{0}(\sigma) \sqrt{T}\right) \sup _{t \in[0, T]}\left\|X_{t}^{P}-x_{0}\right\|_{2 p} \sup _{t \in[0, T]}\left\|V_{1, t}\right\|_{2 p} .
\end{aligned}
$$

We conclude using (3.25), (3.26) $i=1$ and (3.21).

The following Lemma provides the explicit equations solved by the $X$-residual processes, in a form appropriate for tight $L^{p}$ estimates.

Lemma 3.8. Assume $\left(\mathcal{H}_{x_{0}}\right)$ and $(\mathcal{P})$. One has:

$$
\begin{aligned}
\mathrm{d} R_{0, t}^{X}= & \left.\lambda_{t} R_{0, \sigma}\left(X_{t}\right)+\sigma_{t}\left(X_{t}\right) R_{0, t}^{\Lambda}\right] \mathrm{d} W_{t}-\frac{1}{2}\left[v_{t} R_{0, \sigma^{2}}\left(X_{t}\right)+\sigma_{t}^{2}\left(X_{t}\right) R_{0, t}^{V}\right] \mathrm{d} t, R_{0,0}^{X}=0 \\
\mathrm{~d} R_{1, t}^{X}= & {\left[\lambda_{t} R_{1, \sigma}\left(X_{t}\right)+\Lambda_{1, t} R_{0, \sigma}\left(X_{t}\right)+\sigma_{t}\left(X_{t}\right) R_{1, t}^{\Lambda}+\lambda_{t} \sigma_{t}^{(1)} R_{0, t}^{X}\right] \mathrm{d} W_{t} } \\
& -\frac{1}{2}\left[v_{t} R_{1, \sigma^{2}}\left(X_{t}\right)+V_{1, t} R_{0, \sigma^{2}}\left(X_{t}\right)+\sigma_{t}^{2}\left(X_{t}\right) R_{1, t}^{V}+2 v_{t} \sigma_{t} \sigma_{t}^{(1)} R_{0, t}^{X}\right] \mathrm{d} t, R_{1,0}^{X}=0 \\
\mathrm{~d} R_{2, t}^{X}= & \lambda_{t} R_{2, \sigma}\left(X_{t}\right)+\Lambda_{1, t} R_{1, \sigma}\left(X_{t}\right)+\frac{\Lambda_{2, t}}{2} R_{0, \sigma}\left(X_{t}\right)+\sigma_{t}\left(X_{t}\right) R_{2, t}^{\Lambda}+\lambda_{t} \sigma_{t}^{(1)} R_{1, t}^{X}+\lambda_{t} \frac{\sigma_{t}^{(2)}}{2} R_{0, t}^{X}\left(X_{t}+X_{t}^{P}-2 x_{0}\right) \\
& \left.+\Lambda_{1, t} \sigma_{t}^{(1)} R_{0, t}^{X}\right] \mathrm{d} W_{t}-\frac{1}{2}\left[v_{t} R_{2, \sigma^{2}}\left(X_{t}\right)+V_{1, t} R_{1, \sigma^{2}}\left(X_{t}\right)+\frac{V_{2, t}}{2} R_{0, \sigma^{2}}\left(X_{t}\right)+\sigma_{t}^{2}\left(X_{t}\right) R_{2, t}^{V}+2 v_{t} \sigma_{t} \sigma_{t}^{(1)} R_{1, t}^{X}\right. \\
& \left.+v_{t}\left(\left(\sigma_{t}^{(1)}\right)^{2}+\sigma_{t}^{(2)} \sigma_{t}\right) R_{0, t}^{X}\left(X_{t}+X_{t}^{P}-2 x_{0}\right)+2 V_{1, t} \sigma_{t} \sigma_{t}^{(1)} R_{0, t}^{X}\right] \mathrm{d} t, R_{2,0}^{X}=0 .
\end{aligned}
$$

Proof. The verification of these identities is tedious but without mathematical difficulties. For convenience, we detail some computations. To obtain (3.27), start from (1.2)-(1.3) and (1.4) and write:

$$
\begin{aligned}
\mathrm{d} R_{0, t}^{X} & =\left[\sigma_{t}\left(X_{t}\right) \Lambda_{t}-\sigma_{t} \lambda_{t}\right] \mathrm{d} W_{t}-\frac{1}{2}\left[\sigma_{t}^{2}\left(X_{t}\right) V_{t}-\sigma_{t}^{2} v_{t}\right] \mathrm{d} t \\
& =\left[\lambda_{t}\left(\sigma_{t}\left(X_{t}\right)-\sigma_{t}\right)+\sigma_{t}\left(X_{t}\right)\left(\Lambda_{t}-\lambda_{t}\right)\right] \mathrm{d} W_{t}-\frac{1}{2}\left[v_{t}\left(\sigma_{t}^{2}\left(X_{t}\right)-\sigma_{t}^{2}\right)+\sigma_{t}^{2}\left(X_{t}\right)\left(V_{t}-v_{t}\right)\right] \mathrm{d} t \\
& =\left[\lambda_{t} R_{0, \sigma}\left(X_{t}\right)+\sigma_{t}\left(X_{t}\right) R_{0, t}^{\Lambda}\right] \mathrm{d} W_{t}-\frac{1}{2}\left[v_{t} R_{0, \sigma^{2}}\left(X_{t}\right)+\sigma_{t}^{2}\left(X_{t}\right) R_{0, t}^{V}\right] \mathrm{d} t .
\end{aligned}
$$

Similarly for (3.28), using (3.27) and (3.4), we get:

$$
\begin{aligned}
\mathrm{d} R_{1, t}^{X}= & \mathrm{d} R_{0, t}^{X}-\mathrm{d} X_{1, t} \\
= & {\left[\lambda_{t} R_{0, \sigma}\left(X_{t}\right)+\sigma_{t}\left(X_{t}\right) R_{0, t}^{\Lambda}\right] \mathrm{d} W_{t}-\frac{1}{2}\left[v_{t} R_{0, \sigma^{2}}\left(X_{t}\right)+\sigma_{t}^{2}\left(X_{t}\right) R_{0, t}^{V}\right] \mathrm{d} t } \\
& -\left[\left(X_{t}^{P}-x_{0}\right) \sigma_{t}^{(1)} \lambda_{t}+\Lambda_{1, t} \sigma_{t}\right]\left(\mathrm{d} W_{t}-\sigma_{t} \lambda_{t} \mathrm{~d} t\right) \\
= & {\left[\lambda_{t} R_{0, \sigma}\left(X_{t}\right)+\Lambda_{1, t} R_{0, \sigma}\left(X_{t}\right)+\sigma_{t}\left(X_{t}\right) R_{1, t}^{\Lambda}\right] \mathrm{d} W_{t}-\frac{1}{2}\left[v_{t} R_{0, \sigma^{2}}\left(X_{t}\right)+V_{1, t} R_{0, \sigma^{2}}\left(X_{t}\right)+\sigma_{t}^{2}\left(X_{t}\right) R_{1, t}^{V}\right] \mathrm{d} t } \\
& -\left(X_{t}^{P}-x_{0}\right) \sigma_{t}^{(1)} \lambda_{t}\left(\mathrm{~d} W_{t}-\sigma_{t} \lambda_{t} \mathrm{~d} t\right)
\end{aligned}
$$




$$
\begin{aligned}
= & {\left[\lambda_{t} R_{1, \sigma}\left(X_{t}\right)+\Lambda_{1, t} R_{0, \sigma}\left(X_{t}\right)+\sigma_{t}\left(X_{t}\right) R_{1, t}^{\Lambda}+\lambda_{t} \sigma_{t}^{(1)} R_{0, t}^{X}\right] \mathrm{d} W_{t} } \\
& -\frac{1}{2}\left[v_{t} R_{1, \sigma^{2}}\left(X_{t}\right)+V_{1, t} R_{0, \sigma^{2}}\left(X_{t}\right)+\sigma_{t}^{2}\left(X_{t}\right) R_{1, t}^{V}+2 v_{t} \sigma_{t} \sigma_{t}^{(1)} R_{0, t}^{X}\right] \mathrm{d} t .
\end{aligned}
$$

Now consider (3.29). Start from (3.28)-(3.7) and write:

$$
\begin{aligned}
& \mathrm{d} R_{2, t}^{X}=\mathrm{d} R_{1, t}^{X}-\frac{1}{2} \mathrm{~d} X_{2, t} \\
& =\left[\lambda_{t} R_{1, \sigma}\left(X_{t}\right)+\Lambda_{1, t} R_{0, \sigma}\left(X_{t}\right)+\sigma_{t}\left(X_{t}\right) R_{1, t}^{\Lambda}+\lambda_{t} \sigma_{t}^{(1)} R_{0, t}^{X}\right] \mathrm{d} W_{t} \\
& -\frac{1}{2}\left[v_{t} R_{1, \sigma^{2}}\left(X_{t}\right)+V_{1, t} R_{0, \sigma^{2}}\left(X_{t}\right)+\sigma_{t}^{2}\left(X_{t}\right) R_{1, t}^{V}+2 v_{t} \sigma_{t} \sigma_{t}^{(1)} R_{0, t}^{X}\right] \mathrm{d} t \\
& -\frac{1}{2}\left\{\lambda_{t}\left[\left(X_{t}^{P}-x_{0}\right)^{2} \sigma_{t}^{(2)}+2 \sigma_{t}^{(1)} X_{1, t}\right]+2\left(X_{t}^{P}-x_{0}\right) \Lambda_{1, t} \sigma_{t}^{(1)}\right\}\left(\mathrm{d} W_{t}-\sigma_{t} \lambda_{t} \mathrm{~d} t\right) \\
& -\frac{1}{2}\left\{\Lambda_{2, t} \sigma_{t} \mathrm{~d} W_{t}-\left[\left(X_{t}^{P}-x_{0}\right) V_{1, t} \sigma_{t}^{(1)} \sigma_{t}+\left(X_{t}^{P}-x_{0}\right)^{2}\left(\sigma_{t}^{(1)}\right)^{2} v_{t}+\frac{V_{2, t}}{2} \sigma_{t}^{2}\right] \mathrm{d} t\right\} \\
& =\left[\lambda_{t} R_{1, \sigma}\left(X_{t}\right)+\Lambda_{1, t} R_{0, \sigma}\left(X_{t}\right)+\frac{\Lambda_{2, t}}{2} R_{0, \sigma}\left(X_{t}\right)+\sigma_{t}\left(X_{t}\right) R_{2, t}^{\Lambda}+\lambda_{t} \sigma_{t}^{(1)} R_{1, t}^{X}\right] \mathrm{d} W_{t} \\
& -\frac{1}{2}\left[v_{t} R_{1, \sigma^{2}}\left(X_{t}\right)+V_{1, t} R_{0, \sigma^{2}}\left(X_{t}\right)+\frac{V_{2, t}}{2} R_{0, \sigma^{2}}\left(X_{t}\right)+\sigma_{t}^{2}\left(X_{t}\right) R_{2, t}^{V}+2 v_{t} \sigma_{t} \sigma_{t}^{(1)} R_{1, t}^{X}\right] \mathrm{d} t \\
& -\frac{1}{2}\left\{\lambda_{t}\left(X_{t}^{P}-x_{0}\right)^{2} \sigma_{t}^{(2)}+2\left(X_{t}^{P}-x_{0}\right) \Lambda_{1, t} \sigma_{t}^{(1)}\right\}\left(\mathrm{d} W_{t}-\sigma_{t} \lambda_{t} \mathrm{~d} t\right) \\
& +\frac{1}{2}\left[\left(X_{t}^{P}-x_{0}\right) V_{1, t} \sigma_{t}^{(1)} \sigma_{t}+\left(X_{t}^{P}-x_{0}\right)^{2}\left(\sigma_{t}^{(1)}\right)^{2} v_{t}\right] \mathrm{d} t \\
& =\left[\lambda_{t} R_{2, \sigma}\left(X_{t}\right)+\Lambda_{1, t} R_{0, \sigma}\left(X_{t}\right)+\frac{\Lambda_{2, t}}{2} R_{0, \sigma}\left(X_{t}\right)+\sigma_{t}\left(X_{t}\right) R_{2, t}^{\Lambda}+\lambda_{t} \sigma_{t}^{(1)} R_{1, t}^{X}+\lambda_{t} \frac{\sigma_{t}^{(2)}}{2} R_{0, t}^{X}\left(X_{t}+X_{t}^{P}-2 x_{0}\right)\right] \mathrm{d} W_{t} \\
& -\frac{1}{2}\left[v_{t} R_{2, \sigma^{2}}\left(X_{t}\right)+V_{1, t} R_{0, \sigma^{2}}\left(X_{t}\right)+\frac{V_{2, t}}{2} R_{0, \sigma^{2}}\left(X_{t}\right)+\sigma_{t}^{2}\left(X_{t}\right) R_{2, t}^{V}+v_{t}\left(\left(\sigma_{t}^{(1)}\right)^{2}+\sigma_{t}^{(2)} \sigma_{t}\right) R_{0, t}^{X}\left(X_{t}+X_{t}^{P}-2 x_{0}\right)\right. \\
& \left.+2 v_{t} \sigma_{t} \sigma_{t}^{(1)} R_{1, t}^{X}\right] \mathrm{d} t-\left(X_{t}^{P}-x_{0}\right) \Lambda_{1, t} \sigma_{t}^{(1)}\left(\mathrm{d} W_{t}-\sigma_{t} \lambda_{t} \mathrm{~d} t\right)+\frac{1}{2}\left(X_{t}^{P}-x_{0}\right) V_{1, t} \sigma_{t}^{(1)} \sigma_{t} \mathrm{~d} t \\
& =\left[\lambda_{t} R_{2, \sigma}\left(X_{t}\right)+\Lambda_{1, t} R_{1, \sigma}\left(X_{t}\right)+\frac{\Lambda_{2, t}}{2} R_{0, \sigma}\left(X_{t}\right)+\sigma_{t}\left(X_{t}\right) R_{2, t}^{\Lambda}+\lambda_{t} \sigma_{t}^{(1)} R_{1, t}^{X}+\lambda_{t} \frac{\sigma_{t}^{(2)}}{2} R_{0, t}^{X}\left(X_{t}+X_{t}^{P}-2 x_{0}\right)\right. \\
& \left.+\Lambda_{1, t} \sigma_{t}^{(1)} R_{0, t}^{X}\right] \mathrm{d} W_{t}-\frac{1}{2}\left[v_{t} R_{2, \sigma^{2}}\left(X_{t}\right)+V_{1, t} R_{1, \sigma^{2}}\left(X_{t}\right)+\frac{V_{2, t}}{2} R_{0, \sigma^{2}}\left(X_{t}\right)+\sigma_{t}^{2}\left(X_{t}\right) R_{2, t}^{V}+2 v_{t} \sigma_{t} \sigma_{t}^{(1)} R_{1, t}^{X}\right. \\
& \left.+v_{t}\left(\left(\sigma_{t}^{(1)}\right)^{2}+\sigma_{t}^{(2)} \sigma_{t}\right) R_{0, t}^{X}\left(X_{t}+X_{t}^{P}-2 x_{0}\right)+2 V_{1, t} \sigma_{t} \sigma_{t}^{(1)} R_{0, t}^{X}\right] \mathrm{d} t .
\end{aligned}
$$

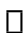

Another intermediate result is the estimates of $R_{n, \sigma}\left(X_{t}\right)$ and $R_{n, \sigma^{2}}\left(X_{t}\right)$. In view of $\left(\mathcal{H}_{x_{0}}\right)$, we have $\left|R_{n, \sigma}\left(X_{t}\right)\right| \leq_{c}\left|X_{t}-x_{0}\right|^{n+1} \mathcal{M}_{1}(\sigma)$ and $\left|R_{n, \sigma^{2}}\left(X_{t}\right)\right| \leq_{c}\left|X_{t}-x_{0}\right|^{n+1} \mathcal{M}_{0}(\sigma) \mathcal{M}_{1}(\sigma)$ for $n=0,1,2$. Combined with (3.24), this readily gives $\forall p \geq 1$ and $\forall j \in\{0,1,2\}$ :

$$
\sup _{t \in[0, T]}\left\|R_{j, \sigma}\left(X_{t}\right)\right\|_{p} \leq_{c}\left(|\sigma|_{\infty} \sqrt{T}\right)^{j+1} \mathcal{M}_{1}(\sigma), \sup _{t \in[0, T]}\left\|R_{j, \sigma^{2}}\left(X_{t}\right)\right\|_{p} \leq_{c}\left(|\sigma|_{\infty} \sqrt{T}\right)^{j+1} \mathcal{M}_{0}(\sigma) \mathcal{M}_{1}(\sigma) .
$$

We are now in a position to estimate the residuals processes, by taking advantage of the explicit representation of Lemma 3.8.

Proposition 3.9. Assume that $\left(\mathcal{H}_{x_{0}}\right)$ and $(\mathcal{P})$ hold. Then for any $p \geq 1$, we have:

$$
\sup _{t \in[0, T]}\left\|R_{j, t}^{X}\right\|_{p} \leq_{c}|\sigma|_{\infty}\left\{\xi_{\text {sup }}^{j+1}+\mathcal{M}_{1}(\sigma)\left(\mathcal{M}_{0}(\sigma)+\xi_{\text {sup }}\right)^{j}\right\} T^{\frac{j}{2}+1}, \forall j \in\{0,1,2\} .
$$


Proof. As before, it is enough to consider $p \geq 2$. We begin with $R_{0, t}^{X}$. Starting from (3.27) and using standard inequalities, it readily follows:

$$
\left\|R_{0, t}^{X}\right\|_{p} \leq{ }_{c} \sqrt{T}\left[\sup _{t \leq T}\left\|R_{0, \sigma}\left(X_{t}\right)\right\|_{p}+|\sigma|_{\infty} \sup _{t \leq T}\left\|R_{0, t}^{\Lambda}\right\|_{p}\right]+T\left[\sup _{t \leq T}\left\|R_{0, \sigma^{2}}\left(X_{t}\right)\right\|_{p}+|\sigma|_{\infty}^{2} \sup _{t \leq T}\left\|R_{0, t}^{V}\right\|_{p}\right] .
$$

We conclude using (3.30)-(3.18)-(3.22). Similarly for $R_{1, t}^{X}$ given in (3.28), we obtain:

$$
\begin{aligned}
\left\|R_{1, t}^{X}\right\|_{p} \leq & \sqrt{T}\left\{\sup _{t \leq T}\left\|R_{1, \sigma}\left(X_{t}\right)\right\|_{p}+\sup _{t \leq T}\left\|\Lambda_{1, t} R_{0, \sigma}\left(X_{t}\right)\right\|_{p}+|\sigma|_{\infty} \sup _{t \leq T}\left\|R_{1, t}^{\Lambda}\right\|_{p}+\mathcal{M}_{1}(\sigma) \sup _{t \leq T}\left\|R_{0, t}^{X}\right\|_{p}\right\} \\
& +T\left\{\sup _{t \leq T}\left\|R_{1, \sigma^{2}}\left(X_{t}\right)\right\|_{p}+\sup _{t \leq T}\left\|V_{1, t} R_{0, \sigma^{2}}\left(X_{t}\right)\right\|_{p}+|\sigma|_{\infty}^{2} \sup _{t \leq T}\left\|R_{1, t}^{V}\right\|\left\|_{p}+|\sigma|_{\infty} \mathcal{M}_{1}(\sigma) \sup _{t \leq T}\right\| R_{0, t}^{X} \|_{p}\right\} .
\end{aligned}
$$

Then, by plugging in the above upper bound the estimates (3.17)-(3.18)-(3.21)-(3.22)-(3.30)-(3.31) $i=0$, we complete the proof of (3.31) for $i=1$. Finally for $R_{2, t}^{X}$, starting from (3.29), we readily have:

$$
\begin{aligned}
\left\|R_{2, t}^{X}\right\|_{p} \leq & \sqrt{T}\left\{\sup _{t \leq T}\left\|R_{2, \sigma}\left(X_{t}\right)\right\|_{p}+\sup _{t \leq T}\left\|\Lambda_{1, t} R_{1, \sigma}\left(X_{t}\right)\right\|_{p}+\left.\sup _{t \leq T}\left\|\Lambda_{2, t} R_{0, \sigma}\left(X_{t}\right)\right\|\right|_{p}+|\sigma|_{\infty} \sup _{t \leq T}\left\|R_{2, t}^{\Lambda}\right\|_{p}\right. \\
& \left.+\mathcal{M}_{1}(\sigma) \sup _{t \leq T}\left\|R_{1, t}^{X}\right\|_{p}+\mathcal{M}_{1}(\sigma) \sup _{t \leq T}\left\|R_{0, t}^{X}\left(X_{t}+X_{t}^{P}-2 x_{0}\right)\right\|_{p}+\mathcal{M}_{1}(\sigma) \sup _{t \leq T}\left\|\Lambda_{1, t} R_{0, t}^{X}\right\|_{p}\right\} \\
& +T\left\{\sup _{t \leq T}\left\|R_{2, \sigma^{2}}\left(X_{t}\right)\right\|_{p}+\sup _{t \leq T}\left\|V_{1, t} R_{1, \sigma^{2}}\left(X_{t}\right)\right\|_{p}+\sup _{t \leq T}\left\|V_{2, t} R_{0, \sigma^{2}}\left(X_{t}\right)\right\|_{p}+|\sigma|_{\infty}^{2} \sup _{t \leq T}\left\|R_{2, t}^{V}\right\|_{p}\right. \\
& \left.+|\sigma|{ }_{\infty} \mathcal{M}_{1}(\sigma) \sup _{t \leq T}\left\|R_{1, t}^{X}\right\|\left\|_{p}+\mathcal{M}_{1}(\sigma) \mathcal{M}_{0}(\sigma) \sup _{t \leq T}\right\| R_{0, t}^{X}\left(X_{t}+X_{t}^{P}-2 x_{0}\right)\left\|_{p}+|\sigma|_{\infty} \mathcal{M}_{1}(\sigma) \sup _{t \leq T}\right\| V_{1, t} R_{0, t}^{X} \|_{p}\right\} .
\end{aligned}
$$

The proof is completed as before using (3.17)-(3.18)-(3.21)-(3.22)-(3.30)-(3.31) $i=0$ and $1 . \square$

3.3. Regularization of the function $h$ by adding a small noise perturbation. As explained in Subsection 3.1, in order to compensate the lack of smoothness of the payoff function $h$ and to overcome some problems of degeneracy in the Malliavin sense, we introduce an extra scalar Brownian motion $\bar{W}$ independent of $W$ and $B$. Let $h$ be a bounded payoff, then define:

$$
h_{\delta}(x)=\mathbb{E}\left[h\left(x+\delta \bar{W}_{T}\right)\right]
$$

for a small parameter $\delta>0$. Clearly the function $h_{\delta}$ is of class $\mathcal{C}_{b}^{\infty}(\mathbb{R})$ thanks to the smooth Gaussian density and remarkably we notice that

$$
h_{\delta}(x)=\mathbb{E}\left[h_{\delta / \sqrt{2}}\left(x+\delta \bar{W}_{\frac{T}{2}}\right)\right] .
$$

In addition $h \in \operatorname{Lip}_{\mathrm{b}}(\mathbb{R}) \Rightarrow \mathrm{h}_{\delta} \in \operatorname{Lip}_{\mathrm{b}}(\mathbb{R})$ and we have $C_{h_{\delta}} \leq C_{h}$ and $L_{h_{\delta}} \leq L_{h}$ (remind (2.2)). The next lemma quantifies the error in terms of $\delta$ induced by considering $h_{\delta}$ instead of $h$ in expectations and sensitivities appearing in Theorem 2.4.

Lemma 3.10. Let $\delta>0$. Assume that $h \in \operatorname{Lip}_{\mathrm{b}}(\mathbb{R})$ and that $\left(\mathcal{H}_{x_{0}}\right)$ is satisfied. Then we have:

$$
\begin{aligned}
\left|\mathbb{E}\left[h\left(X_{T}\right)\right]-\mathbb{E}\left[h_{\delta}\left(X_{T}\right)\right]\right|+\left|\mathbb{E}\left[h\left(X_{T}^{P}\right)\right]-\mathbb{E}\left[h_{\delta}\left(X_{T}^{P}\right)\right]\right| & \leq_{c} L_{h} \delta \sqrt{T}, \\
\left|\partial_{x^{i}}^{i} \mathbb{E}\left[h\left(X_{T}^{P}+x\right)\right]\right|_{x=0}-\left.\partial_{x^{i}}^{i} \mathbb{E}\left[h_{\delta}\left(X_{T}^{P}+x\right)\right]\right|_{x=0} \mid & \leq_{c} L_{h} \frac{\delta \sqrt{T}}{\left(\int_{0}^{T} \sigma_{t}^{2} v_{t} \mathrm{~d} t\right)^{i / 2}}, \forall i \geq 1 .
\end{aligned}
$$


Proof. The first estimate directly follows from the Lipschitz property of $h$. For the second one, write:

$$
\mathbb{E}\left[h_{\delta}\left(X_{T}^{P}+x\right)\right]=\int_{\mathbb{R}} \mathbb{E}\left[h\left(y-\frac{\int_{0}^{T} \sigma_{t}^{2} v_{t} \mathrm{~d} t}{2}+\delta \bar{W}_{T}\right)\right] \frac{e^{-\frac{\left(y-x^{2}\right.}{2 \int_{0}^{T} \sigma_{t}^{2} v_{t} \mathrm{~d} t}}}{\sqrt{2 \pi \int_{0}^{T} \sigma_{t}^{2} v_{t} \mathrm{~d} t}} \mathrm{~d} y,
$$

to obtain:

$$
\begin{aligned}
& \left.\partial_{x^{i}}^{i} \mathbb{E}\left[h\left(X_{T}^{P}+x\right)\right]\right|_{x=0}-\left.\partial_{x^{i}}^{i} \mathbb{E}\left[h_{\delta}\left(X_{T}^{P}+x\right)\right]\right|_{x=0} \\
= & \left.\int_{\mathbb{R}} \mathbb{E}\left[h\left(y-\frac{\int_{0}^{T} \sigma_{t}^{2} v_{t} \mathrm{~d} t}{2}+\delta \bar{W}_{T}\right)-h\left(y-\frac{\int_{0}^{T} \sigma_{t}^{2} v_{t} \mathrm{~d} t}{2}\right)\right] \partial_{x^{i}}^{i}\left\{\frac{e^{-\frac{(y-x)^{2}}{2 \int_{0}^{T} \sigma_{t}^{2} v_{t} \mathrm{~d} t}}}{\sqrt{2 \pi \int_{0}^{T} \sigma_{t}^{2} v_{t} \mathrm{~d} t}}\right\}\right|_{x=0} \mathrm{~d} y .
\end{aligned}
$$

Then we easily complete the proof using again that $h$ is Lipschitz and standard upper bounds for the derivatives of the Gaussian density.

In view of the magnitudes of the coefficients $C_{i, T}^{l}, C_{i, T}^{s}$ and $C_{i, T}^{l s}$ defined in Theorem 2.4, Lemma 3.10 readily yields

$$
\begin{aligned}
\mid \text { Error }_{3, h} \mid= & \left|\mathbb{E}\left[h\left(X_{T}\right)\right]-\mathbb{E}\left[h\left(X_{T}^{P}\right)\right]-\sum_{i=1}^{6} \eta_{i, T} \mathcal{G}_{i}^{h}\right| \\
\leq & \left|\mathbb{E}\left[h\left(X_{T}\right)\right]-\mathbb{E}\left[h_{\delta}\left(X_{T}\right)\right]\right|+\left|\mathbb{E}\left[h_{\delta}\left(X_{T}^{P}\right)\right]-\mathbb{E}\left[h\left(X_{T}^{P}\right)\right]\right| \\
& +\sum_{i=1}^{6}\left|\eta_{i, T}\right|\left|\mathcal{G}_{i}^{h}-\mathcal{G}_{i}^{h_{\delta}}\right|+\mid \text { Error }_{3, h_{\delta}}\left|\leq_{c} L_{h} \delta \sqrt{T}+\right| \text { Error }_{3, h_{\delta}} \mid .
\end{aligned}
$$

Assume now without loss of generality that $\xi_{\text {sup }} \neq 0(\operatorname{valid}$ under $(\mathcal{P}))$. We may prove the advertised error estimate (2.4) provided that we choose

$$
\delta=|\sigma|_{\infty}\left[\xi_{\text {sup }}^{3}+\mathcal{M}_{1}(\sigma)\left(\mathcal{M}_{0}(\sigma)+\xi_{\text {sup }}\right)^{2}\right] T^{\frac{3}{2}}
$$

and that, for such a $\delta$, we show

$$
\mid \text { Error }\left._{3, h_{\delta}}\left|\leq_{c} L_{h}\right| \sigma\right|_{\infty}\left[\xi_{\text {sup }}^{3}+\mathcal{M}_{1}(\sigma)\left(\mathcal{M}_{0}(\sigma)+\xi_{\text {sup }}\right)^{2}\right] T^{2} .
$$

This is the purpose of the next subsection, where we will consider the representation (3.15) for Error $_{3, h_{\delta}}$.

3.4. Malliavin integration by part formula and proof of estimate (3.35). We write $W_{t}=\int_{0}^{t} \rho_{s} \mathrm{~d} B_{s}+\int_{0}^{t} \sqrt{1-\rho_{s}^{2}} \mathrm{~d} B_{s}^{\perp}$ where $\left(B_{t}^{\perp}\right)_{0 \leq t \leq T}$ is a Brownian motion independent of $B_{t}$ and we consider the calculus of stochastic variations w.r.t. the three-dimensional Brownian motion $\left(B, B^{\perp}, \bar{W}\right)$, the Malliavin derivative operator w.r.t. $B, B^{\perp}$ and $\bar{W}$ being respectively denoted by $D()=.\left(D_{t}^{1}(.), D_{t}^{2}(.), D_{t}^{3}(.)\right)_{t \in[0, T]}$. For the second derivatives, we use the obvious notation $\left(D^{i, j}(.)\right)_{s, t \in[0, T]}$ for $i, j \in\{1,2,3\}$ and so on for the higher derivatives. We freely adopt the notations of [Nua06] for the Sobolev space $\mathbb{D}^{k, p}$ associated to the norm $\|.\|_{k, p}$.

In the following lemma, we provide estimates of the Malliavin derivatives of $X_{t}^{P}, X_{1, t}$ and $X_{2, t}$ for $t \in[0, T]$.

Lemma 3.11. Assume that $\left(\mathcal{H}_{x_{0}}\right)$ and $(\mathcal{P})$ hold. Then, $\forall t \in[0, T], X_{t}^{P}, X_{1, t}, X_{2, t}, V_{1, t}$, and $V_{2, t} \in \mathbb{D}^{3, \infty}$. Moreover, we have the following estimates, $\forall p \geq 1$, uniformly in $q, r, s, t \in[0, T]$ :

$$
\left\|D_{s}^{1} X_{t}^{P}\right\|_{p}+\left\|D_{s}^{2} X_{t}^{P}\right\|_{p} \leq_{c}|\sigma|_{\infty}
$$




$$
\begin{aligned}
\left\|D_{s}^{1} \Lambda_{n, t}\right\|_{p}+\left\|D_{s}^{1} V_{n, t}\right\|_{p} & \leq_{c} \xi_{\text {sup }}^{n} T^{\frac{n-1}{2}}, \forall n \in\{1,2\} \\
\left\|D_{s}^{1} X_{n, t}||_{p}+\right\| D_{s}^{2} X_{n, t} \|_{p} & \leq_{c}|\sigma|_{\infty}\left[\xi_{\text {sup }}^{n}+\mathcal{M}_{1}(\sigma)\left(\mathcal{M}_{0}(\sigma)+\xi_{\text {sup }}\right)^{n-1}\right] T^{\frac{n}{2}}, \forall n \in\{1,2\} \\
\left\|D_{r, s}^{1,1} \Lambda_{2, t}\right\|_{p}+\left\|D_{r, s}^{1,1} V_{2, t}\right\|_{p} & \leq_{c} \xi_{\text {sup }}^{2} \\
\sum_{i, j \in\{1,2\}}\left\|D_{r, s}^{i, j} X_{n, t}\right\|_{p} & \leq_{c}|\sigma|_{\infty}\left[\xi_{\text {sup }}^{n}+\mathcal{M}_{1}(\sigma)\left(\mathcal{M}_{0}(\sigma)+\xi_{\text {sup }}\right)^{n-1}\right] T^{\frac{n-1}{2}}, \forall n \in\{1,2\} \\
\sum_{i, j, k \in\{1,2\}}\left\|D_{q, r, s}^{i, j, k} X_{2, t}\right\|_{p} & \leq_{c}|\sigma|_{\infty}\left[\xi_{\text {sup }}^{2}+\mathcal{M}_{1}(\sigma)\left(\mathcal{M}_{0}(\sigma)+\xi_{\text {sup }}\right)\right] .
\end{aligned}
$$

Proof. All the 5 variables are clearly in $\mathbb{D}^{3, \infty}$ because they are defined as iteration and smooth composition of Wiener integrals (see (1.4)-(3.4)-(3.7)-(3.5)-(3.8)). Then the calculus of the derivatives and the $L^{p}$-estimates is classical and is left to the reader. In particular, all the derivatives w.r.t. the third Brownian motion $\bar{W}$ are null as well as the derivatives of $V_{1, t}, \Lambda_{1, t}, V_{2, t}$ and $\Lambda_{2, t}$ w.r.t. $B^{\perp}$.

We now state the crucial result related to integration by parts formulas which is proved later.

Proposition 3.12. Assume $\left(\mathcal{H}_{x_{0}}\right)$ and $(\mathcal{P})$. For any $\eta \in[0,1]$, we define the random variable $G_{\delta}^{\eta}:=X_{T}^{P}+\eta\left(X_{1, T}+\frac{X_{2, T}}{2}\right)+\delta \bar{W}_{T / 2}$. Let $i \in\{2,3\}$, for any $Y$ in $\mathbb{D}^{i-1, \infty}$, there exist random variables $Y_{i, \eta} \in \cap_{p \geq 1} L^{p}$ such that

$$
\mathbb{E}\left[Y h_{\delta / \sqrt{2}}^{(i)}\left(G_{\delta}^{\eta}\right)\right]=\mathbb{E}\left[Y_{i, \eta} h_{\delta / \sqrt{2}}^{(1)}\left(G_{\delta}^{\eta}\right)\right],
$$

where, for any $p \geq 1$,

$$
\sup _{\eta \in[0,1]}\left\|Y_{i, \eta}\right\|_{p} \leq_{c}\|Y\|_{i-1, p+\frac{1}{2}}\left(\int_{0}^{T} \sigma_{t}^{2} v_{t} \mathrm{~d} t\right)^{-\frac{(i-1)}{2}}
$$

We are now in a position to achieve the proof of (3.35). Consider Error $_{3, h_{\delta}}$ explicitly written in (3.15). The first term of (3.15) is easily handled using (3.31) $j=2$, in addition we notice that $\left|h_{\delta / \sqrt{2}}^{(1)}\right|_{\infty} \leq L_{h}$. For the second term of (3.15), use (3.33) and apply Proposition 3.12 with $Y=X_{1, T} X_{2, T}+\frac{X_{2, T}^{2}}{4}$ : thus using (3.26)-(3.38), we obtain:

$$
\begin{aligned}
& \quad\left|\mathbb{E}\left[h_{\delta}^{(2)}\left(X_{T}^{P}\right)\left(X_{1, T} X_{2, T}+\frac{X_{2, T}^{2}}{4}\right)\right]\right|=\left|\mathbb{E}\left[h_{\delta / \sqrt{2}}^{(2)}\left(G_{\delta}^{0}\right) Y\right]\right|=\left|\mathbb{E}\left[h_{\delta / \sqrt{2}}^{(1)}\left(G_{\delta}^{0}\right) Y_{1,0}\right]\right| \\
& \leq_{c} L_{h}\|Y\|_{1,2}\left(\int_{0}^{T} v_{t} \sigma_{t}^{2} \mathrm{~d} t\right)^{-1 / 2} \leq_{c} L_{h}|\sigma|_{\infty}\left[\xi_{\text {sup }}^{3}+\mathcal{M}_{1}(\sigma)\left(\mathcal{M}_{0}(\sigma)+\xi_{\text {sup }}\right)^{2}\right] T^{2} .
\end{aligned}
$$

The last term of (3.15) is handled similarly; apply Proposition 3.12 with $Y=\left(X_{1, T}+\frac{X_{2, T}}{2}\right)^{3}$ and use (3.26)-(3.38)-(3.40)-(3.41) to obtain the announced result. To summarize, the proof of Theorem 2.4 is now complete provided that we establish Proposition 3.12, which is done in the following subsection.

3.5. Proof of Proposition 3.12. $X_{T}^{P}$ is a non degenerate random variable with Malliavin covariance matrix equal to $\int_{0}^{T} \sigma_{t}^{2} v_{t} \mathrm{~d} t>0$ thanks to $\left(\mathcal{H}_{x_{0}}\right)$ but $G^{\eta}=X_{T}^{P}+\eta\left(X_{1, T}+\frac{X_{2, T}}{2}\right)$ may be degenerate for $\eta>0$ and this is the second reason to have introduced the small perturbation $\delta \bar{W}_{T / 2}$. Consider the random variable $G_{\delta}^{\eta}=G^{\eta}+\delta \bar{W}_{T / 2}$ defined in Proposition 3.12: clearly 
it belongs to $\mathbb{D}^{3, \infty}$ (even to $\mathbb{D}^{\infty, \infty}$ but higher regularity will not be used here). Its Malliavin covariance matrix is invertible since

$$
\gamma_{G_{\delta}^{\eta}}=\sum_{i=1}^{2} \int_{0}^{T}\left(D_{t}^{i} G^{\eta}\right)^{2} \mathrm{~d} t+\delta^{2} \frac{T}{2}=\gamma_{G^{\eta}}+\delta^{2} \frac{T}{2} \geq \delta^{2} \frac{T}{2}>0 .
$$

Then in view of (3.34)-(3.36)-(3.38)-(3.40)-(3.41) it readily comes for $i \in\{1,2\}$ and any $p \geq 1$ :

$$
\left\|\left(D^{1} G_{\delta}^{\eta}, D^{2} G_{\delta}^{\eta}, D^{3} G_{\delta}^{\eta}\right)\right\|_{i, p} \leq_{c}|\sigma|_{\infty} \sqrt{T} .
$$

Hence, applying [Nua06, Proposition 1.5.6 and Proposition 2.1.4] and using (3.44) we get the existence of $Y_{2, \eta}$ and $Y_{3, \eta}$ such that for any $i \in\{2,3\}$ and any $p \geq 1$ :

$$
\begin{aligned}
\left\|Y_{i, \eta}\right\|_{p} & \leq_{c}\|Y\|_{i-1, p+\frac{1}{2}}\left\|D G_{\delta}^{\eta}\right\|_{i-1,2 p(2 p+1)}^{i-1}\left\|\gamma_{G_{\delta}^{\eta}}^{-1}\right\|_{i-1,2 p(2 p+1)}^{i-1} \\
& \leq_{c}\|Y\|_{i-1, p+\frac{1}{2}}\left(|\sigma|_{\infty} \sqrt{T}\right)^{i-1}\left\|\gamma_{G_{\delta}^{\eta}}^{-1}\right\|_{i-1,2 p(2 p+1)}^{i-1} .
\end{aligned}
$$

It remains to finely estimate the norms related to the inverse of the Malliavin covariance matrix $\gamma_{G_{\delta}^{\eta}}$. First notice that using the definitions of $G^{\eta}$ and $\gamma_{G^{\eta}}$ we have:

$$
\begin{aligned}
\gamma_{G^{\eta}}= & \int_{0}^{T} \sigma_{t}^{2} v_{t} \mathrm{~d} t+\eta^{2} \int_{0}^{T}\left|D_{t}\left(X_{1, T}+\frac{X_{2, T}}{2}\right)\right|^{2} \mathrm{~d} t \\
& +2 \eta \int_{0}^{T} \sigma_{t} \sqrt{v_{t}}\left(\rho_{t}, \sqrt{1-\rho_{t}^{2}}, 0\right) \cdot D_{t}\left(X_{1, T}+\frac{X_{2, T}}{2}\right) \mathrm{d} t .
\end{aligned}
$$

Therefore, from estimates $(3.36)-(3.38),\left(\mathcal{H}_{x_{0}}\right)$ and $(\mathcal{P})$, we easily obtain

$$
\sup _{\eta \in[0,1]} \| \gamma_{G^{\eta}}-\int_{0}^{T} \sigma_{t}^{2} v_{t} \mathrm{~d} t||_{p} \leq_{c}|\sigma|_{\infty}^{2}\left(\xi_{\text {sup }}+\mathcal{M}_{1}(\sigma)\right) T^{\frac{3}{2}},
$$

for any $p \geq 1$. This intermediate estimate allows to prove the next lemma.

Lemma 3.13. Assume $\left(\mathcal{H}_{x_{0}}\right)$ and $(\mathcal{P})$. Then $\left(\gamma_{G_{\delta}^{\eta}}\right)^{-1} \in \mathbb{D}^{2, \infty}$ and we have for any $p \geq 1$ :

$$
\begin{gathered}
\sup _{\eta \in[0,1]}\left\|\left(\gamma_{G_{\delta}^{\eta}}\right)^{-1}\right\|_{p} \leq_{c}\left(\int_{0}^{T} \sigma_{t}^{2} v_{t} \mathrm{~d} t\right)^{-1} \\
\sup _{t \in[0, T], \eta \in[0,1]} \sum_{i \in\{1,2\}}\left\|D_{t}^{i}\left(\gamma_{G_{\delta}^{\eta}}\right)^{-1}\right\|_{p} \leq_{c}\left(\mathcal{M}_{1}(\sigma)+\xi_{\text {sup }}\right)\left(\int_{0}^{T} \sigma_{t}^{2} v_{t} \mathrm{~d} t\right)^{-1} \\
\sup _{s, t \in[0, T], \eta \in[0,1]} \sum_{i, j \in\{1,2\}}\left\|D_{s, t}^{i, j}\left(\gamma_{G_{\delta}^{\eta}}\right)^{-1}\right\|_{p} \leq_{c}\left[\xi_{\text {sup }}^{2}+\mathcal{M}_{1}(\sigma)\left(\mathcal{M}_{0}(\sigma)+\xi_{\text {sup }}\right)\right]\left(\int_{0}^{T} \sigma_{t}^{2} v_{t} \mathrm{~d} t\right)^{-1}
\end{gathered}
$$

Proof. For the sake of brevity, we only prove (3.47) and (3.48) because there is no extra difficulties for (3.49). For (3.47), we have for any $p \geq 1$ and $q \geq 1$ :

$$
\begin{aligned}
\mathbb{E}\left[\left(\gamma_{G_{\delta}^{\eta}}\right)^{-p}\right] & =\mathbb{E}\left[\left(\gamma_{G_{\delta}^{\eta}}\right)^{-p} \mathbb{1}_{\gamma_{G} \eta \frac{1}{2} \int_{0}^{T} \sigma_{t}^{2} v_{t} \mathrm{~d} t}\right]+\mathbb{E}\left[\left(\gamma_{G_{\delta}^{\eta}}\right)^{-p} \mathbb{1}_{\gamma_{G^{\eta}}>\frac{1}{2} \int_{0}^{T} \sigma_{t}^{2} v_{t} \mathrm{~d} t}\right] \\
& \leq\left(\delta^{2} \frac{T}{2}\right)^{-p} \mathbb{P}\left(\int_{0}^{T} \sigma_{t}^{2} v_{t} \mathrm{~d} t-\gamma_{G^{\eta}} \geq \frac{\int_{0}^{T} \sigma_{t}^{2} v_{t} \mathrm{~d} t}{2}\right)+\left(\frac{1}{2} \int_{0}^{T} \sigma_{t}^{2} v_{t} \mathrm{~d} t\right)^{-p}
\end{aligned}
$$




$$
\begin{aligned}
& \leq_{c}\left(\delta^{2} T\right)^{-p}\left(\int_{0}^{T} \sigma_{t}^{2} v_{t} \mathrm{~d} t\right)^{-q}\left\|\gamma_{G^{\eta}}-\int_{0}^{T} \sigma_{t}^{2} v_{t} \mathrm{~d} t\right\|_{q}^{q}+\left(\int_{0}^{T} \sigma_{t}^{2} v_{t} \mathrm{~d} t\right)^{-p} \\
& \leq_{c}\left(\int_{0}^{T} \sigma_{t}^{2} v_{t} \mathrm{~d} t\right)^{-p}\left[\left(\delta^{2} T\right)^{-p}\left(\int_{0}^{T} \sigma_{t}^{2} v_{t} \mathrm{~d} t\right)^{-q+p}\left\|\gamma_{G^{\eta}}-\int_{0}^{T} \sigma_{t}^{2} v_{t} \mathrm{~d} t\right\|_{q}^{q}+1\right],
\end{aligned}
$$

where we have used the Markov inequality at the third line. Then choosing $q=6 p$ and using (3.34)-(3.46), we readily obtain:

$$
\begin{aligned}
\left\|\left(\gamma_{G_{\delta}^{\eta}}\right)^{-1}\right\|_{p} \leq & \left(\int_{0}^{T} \sigma_{t}^{2} v_{t} \mathrm{~d} t\right)^{-1} \\
& \times\left[\left(|\sigma|_{\infty}\left[\xi_{\text {sup }}^{3}+\mathcal{M}_{1}(\sigma)\left(\mathcal{M}_{0}(\sigma)+\xi_{\text {sup }}\right)^{2}\right] T^{2}\right)^{-2}\left(T|\sigma|_{\infty}^{2}\right)^{-5}\left(|\sigma|_{\infty}^{2}\left[\xi_{\text {sup }}+\mathcal{M}_{1}(\sigma)\right] T^{\frac{3}{2}}\right)^{6}+1\right] \\
\leq & \left(\int_{0}^{T} \sigma_{t}^{2} v_{t} \mathrm{~d} t\right)^{-1} .
\end{aligned}
$$

The inequality (3.48) is a straightforward consequence of [Nua06, Lemma 2.1.6], which writes

$$
D_{t}^{i}\left(\gamma_{G_{\delta}^{\eta}}\right)^{-1}=-\frac{D_{t}^{i} \gamma_{G_{\delta}^{\eta}}}{\gamma_{G_{\delta}^{\eta}}^{2}}=-2 \frac{\int_{0}^{T}\left[D_{u}^{1} G^{\eta} D_{t, u}^{i, 1} G^{\eta}+D_{u}^{2} G^{\eta} D_{t, u}^{i, 2} G^{\eta}\right] \mathrm{d} u}{\gamma_{G_{\delta}^{\eta}}^{2}}, i \in\{1,2\}, t \in[0, T] .
$$

Then using (3.36)-(3.38)-(3.40)-(3.47) we readily get, $\forall p \geq 1$,

$$
\sup _{t \in[0, T]}\left\|D_{t}^{i}\left(\gamma_{G_{\delta}^{\eta}}\right)^{-1}\right\|_{p} \leq_{c} T|\sigma|_{\infty}^{2}\left(\mathcal{M}_{1}(\sigma)+\xi_{\text {sup }}\right)\left(\int_{0}^{T} \sigma_{t}^{2} v_{t} \mathrm{~d} t\right)^{-2}
$$

which leads to (3.48).

Now plug (3.47)-(3.48)-(3.49) in (3.45) to complete the proof of (3.43) and thus Proposition 3.12 .

4. Expansion formulas for the implied volatility. Now we have at hand an expansion formula for the price $\mathbb{E}\left[h\left(X_{T}\right)\right]$ (recall that we consider discounted asset so that risk-free rate is set to 0) with an expansion which takes the form of Black-Scholes model price plus weighted sensitivities, we are in a position to derive expansion formulas for the (Black-Scholes) implied volatilities. Indeed we know that direct implied volatility expansions may be more accurate than the corresponding price formulas, moreover they are usually simpler.

This derivation follows a more or less standard computational routine (see for instance [BG12, LPP14]) by taking advantage of the relations between sensitivities w.r.t. the log-spot and those w.r.t. volatility. Computations being quite standard (but tedious), we only state the approximation formulas on implied volatilities without proof and we refer to [Bom13] for the full details.

Actually we provide two expansions of implied volatility, one when the local volatility is computed at the log-spot $x_{0}$ (see Subsection 4.2), one when it is computed at the average point between spot and strike, which is known to be more symmetric and accurate (see Subsection 4.3). Remind that the asset price model is martingale, and as explained in Subsection 2.3, this allows to apply our main theorem to the Call payoff $h(x)=\left(e^{x}-K\right)_{+}$.

\subsection{Notations.}

Call options. We denote by Call $\left(S_{0}, T, K\right)$ the price at time 0 of a Call option with spot $S_{0}=$ $e^{x_{0}}$, maturity $T$ and strike $K$, written on the asset $S=e^{X}$ that is $\operatorname{Call}\left(S_{0}, T, K\right)=\mathbb{E}\left[\left(e^{X_{T}}-K\right)_{+}\right]$. As usual, ATM (At The Money) Call refers to $S_{0} \approx K$, ITM (In The Money) to $S_{0} \gg K$, OTM (Out The Money) to $S_{0} \ll K$. 
Black-Scholes Call price function. For the sake of completeness, we give the Black-Scholes Call price function depending on $\log$-spot $x$, total variance $y$ and log-strike $k$ :

$$
\operatorname{Call}^{\mathrm{BS}}(x, y, k)=e^{x} \mathcal{N}\left(d_{1}(x, y, k)\right)-e^{k} \mathcal{N}\left(d_{2}(x, y, k)\right)
$$

where:

$$
\begin{array}{rlrl}
\mathcal{N}(x) & =\int_{-\infty}^{x} \mathcal{N}^{\prime}(u) \mathrm{d} u, & \mathcal{N}^{\prime}(u)=\frac{e^{-u^{2} / 2}}{\sqrt{2 \pi}}, \\
d_{1}(x, y, k)=\frac{x-k}{\sqrt{y}}+\frac{1}{2} \sqrt{y}, & d_{2}(x, y, k)=d_{1}(x, y, k)-\sqrt{y} .
\end{array}
$$

In the following, $x_{0}=\log \left(S_{0}\right)$ will represent the $\log$-spot, $k=\log (K)$ the $\log$-strike, $x_{\text {avg }}=\left(x_{0}+\right.$ $k) / 2=\log \left(\sqrt{S_{0} K}\right)$ the mid-point between the log-spot and the log-strike, $m=x_{0}-k=\log \left(S_{0} / K\right)$ the log-moneyness. The value $\mathrm{Call}^{\mathrm{BS}}\left(x_{0}, \int_{0}^{T} \sigma_{t}^{2} v_{t} \mathrm{~d} t, k\right)=\mathbb{E}\left[\left(e^{X_{T}^{P}}-e^{k}\right)_{+}\right]$equals $\operatorname{Call}\left(S_{0}, T, K\right)=$ $\mathbb{E}\left[\left(e^{X_{T}}-K\right)_{+}\right]$when $\mathcal{M}_{1}(\sigma)=\xi_{\text {sup }}=0$. For $(x, T, k)$ given, the implied Black-Scholes volatility of a price $\operatorname{Call}\left(e^{x}, T, e^{k}\right)$ is the unique non-negative volatility parameter $\sigma_{\mathrm{I}}(x, T, k)$ such that:

$$
\operatorname{Call}^{\mathrm{BS}}\left(x, \sigma_{\mathrm{I}}^{2}(x, T, k) T, k\right)=\operatorname{Call}\left(e^{x}, T, e^{k}\right) .
$$

Quadratic mean of the volatility on $[0, T]$. For any spatial point $z \in \mathbb{R}$, we denote by $\bar{\sigma}_{z}$ the quadratic mean on $[0, T]$ of $\left(\sigma_{t}(z) \sqrt{v_{t}}\right)_{t \in[0, T]}$ defined by:

$$
\bar{\sigma}_{z}=\sqrt{\frac{1}{T} \int_{0}^{T} \sigma_{t}^{2}(z) v_{t} \mathrm{~d} t .}
$$

This notation is frequently used for the points $x_{0}$ and $x_{a v g}$. When applied in $x_{0}$, we simply write $\bar{\sigma}$ if unambiguous.

4.2. Implied volatility expansions at spot. We introduce new corrective coefficients useful for the implied volatility expansions:

Definition 4.1. Assume $\left(\mathcal{H}_{x_{0}}\right)$. We define the following corrective coefficients:

$$
\begin{aligned}
\gamma_{0 a, T}= & \bar{\sigma}+\frac{C_{1, T}^{s}}{4 \bar{\sigma} T}, \\
\gamma_{0 b, T}= & \frac{C_{5, T}^{l}}{2 \bar{\sigma}^{3} T^{2}}-\frac{C_{6, T}^{l}}{4 \bar{\sigma}^{3} T^{2}}-\frac{3 C_{6, T}^{l}}{\bar{\sigma}^{5} T^{3}}+\frac{C_{2, T}^{s}}{8 \bar{\sigma} T}-\frac{C_{2, T}^{s}}{2 \bar{\sigma}^{3} T^{2}}-\frac{C_{3, T}^{s}}{16 \bar{\sigma} T}-\frac{C_{3, T}^{s}}{4 \bar{\sigma}^{3} T^{2}}+\frac{3\left(C_{1, T}^{s}\right)^{2}}{8 \bar{\sigma}^{5} T^{3}} \\
& +\frac{C_{7, T}^{l s}}{\bar{\sigma}^{3} T^{2}}-\frac{C_{4, T}^{l s}}{8 \bar{\sigma} T}-\frac{3 C_{4, T}^{l s}}{2 \bar{\sigma}^{3} T^{2}}-\frac{C_{5, T}^{l s}}{8 \bar{\sigma} T}-\frac{C_{5, T}^{l s}}{2 \bar{\sigma}^{3} T^{2}}-\frac{C_{6, T}^{l s}}{2 \bar{\sigma}^{3} T^{2}}+\frac{\left(C_{1, T}^{l} C_{1, T}^{s}\right)}{8 \bar{\sigma}^{3} T^{2}}+\frac{3\left(C_{1, T}^{l} C_{1, T}^{s}\right)}{2 \bar{\sigma}^{5} T^{3}}, \\
\gamma_{1 a, T}= & -\frac{C_{1, T}^{l}}{\bar{\sigma}^{3} T^{2}}-\frac{C_{1, T}^{s}}{2 \bar{\sigma}^{3} T^{2}}, \\
\gamma_{1 b, T}= & -\frac{C_{2, T}^{s}}{2 \bar{\sigma}^{3} T^{2}}+\frac{3\left(C_{1, T}^{s}\right)^{2}}{8 \bar{\sigma}^{5} T^{3}}-\frac{C_{2, T}^{l s}+C_{3, T}^{l s}}{2 \bar{\sigma}^{3} T^{2}}-\frac{C_{4, T}^{l s}}{2 \bar{\sigma}^{3} T^{2}}-\frac{C_{6, T}^{l s}}{4 \bar{\sigma}^{3} T^{2}}+\frac{3\left(C_{1, T}^{l} C_{1, T}^{s}\right)}{4 \bar{\sigma}^{5} T^{3}}, \\
\gamma_{2, T}= & \frac{C_{3, T}^{l}}{\bar{\sigma}^{5} T^{3}}-\frac{3 C_{4, T}^{l}}{\bar{\sigma}^{5} T^{3}}+6 \frac{C_{6, T}^{l}}{\bar{\sigma}^{7} T^{4}}+\frac{C_{2, T}^{s}}{2 \bar{\sigma}^{5} T^{3}}+\frac{C_{3, T}^{s}}{4 \bar{\sigma}^{5} T^{3}}-\frac{3\left(C_{1, T}^{s}\right)^{2}}{4 \bar{\sigma}^{7} T^{4}}+\frac{C_{2, T}^{l s}+C_{3, T}^{l s}}{\bar{\sigma}^{5} T^{3}} \\
& +\frac{3 C_{4, T}^{l s}}{2 \bar{\sigma}^{5} T^{3}}+\frac{C_{5, T}^{l s}}{2 \bar{\sigma}^{5} T^{3}}+\frac{C_{6, T}^{l s}}{2 \bar{\sigma}^{5} T^{3}}-\frac{3\left(C_{1, T}^{l} C_{1, T}^{s}\right)}{\bar{\sigma}^{7} T^{4}},
\end{aligned}
$$


where $\left(C_{i, T}^{l}\right)_{1 \leq i \leq 4}-\left(C_{i, T}^{s}\right)_{1 \leq i \leq 3}-\left(C_{i, T}^{l s}\right)_{1 \leq i \leq 6}$ are defined in Theorem 2.4 and $C_{5, T}^{l}-C_{6, T}^{l}-C_{7, T}^{l s}$ are defined by:

$$
\begin{aligned}
& C_{5, T}^{l}=\omega\left(\sigma^{2} v,\left(\left(\sigma^{(1)}\right)^{2}+\sigma \sigma^{(2)}\right) v, \sigma^{2} v\right)_{0}^{T}, \quad C_{6, T}^{l}=\omega\left(\sigma^{2} v, \sigma \sigma^{(1)} v, \sigma \sigma^{(1)} v, \sigma^{2} v\right)_{0}^{T}, \\
& C_{7, T}^{l s}=\omega\left(\rho \xi \sigma v, \sigma \sigma^{(1)}, \sigma^{2} v\right)_{0}^{T} .
\end{aligned}
$$

Our first implied volatility expansion states that the implied volatility writes as a quadratic function w.r.t. the log-moneyness $m$ with coefficients defined by $\gamma$.

Theorem 4.2 (3rd order expansion of the implied volatility). Assume $\left(\mathcal{H}_{x_{0}}\right)$ and $(\mathcal{P})$. We have:

$$
\sigma_{\mathrm{I}}\left(x_{0}, T, k\right)=\gamma_{0 a, T}+\gamma_{0 b, T}+\left(\gamma_{1 a, T}+\gamma_{1 b, T}\right) m+\gamma_{2, T} m^{2}+\operatorname{Error}_{3, x_{0}}^{\mathrm{I}} .
$$

In addition, for any $C_{m}>0$ and any $|m| \leq C_{m}|\sigma|_{\infty} \sqrt{T}$, the error is estimated as

$$
\mid \text { Error }\left._{3, x_{0}}^{\mathrm{I}}\left|\leq_{c}\right| \sigma\right|_{\infty}\left[\xi_{\text {sup }}^{3}+\mathcal{M}_{1}(\sigma)\left(\mathcal{M}_{0}(\sigma)+\xi_{\text {sup }}\right)^{2}\right] T^{\frac{3}{2}},
$$

where the generic constant depends in an increasing way of $C_{m}$ (but not $m$ ).

Several remarks and corollaries follow from the above result.

$\triangleright$ Short maturity skew and smile behaviors. We analyse the behavior of the approximation formula (4.4) at the money (i.e. $m \approx 0$ ) and for short maturity (i.e. $T \ll 1$ ). In view of $(4.4)$ and the various coefficients $C^{l}, C^{s}, C^{l s}$ and $\gamma$ (see Definition 4.1 and Theorem 2.4), assuming that $\sigma_{t}$, $\sigma_{t}^{(1)}$ and $\sigma_{t}^{(2)}$ are continuous at $t=0$, we obtain the level, the slope and the curvature ATM:

$$
\begin{gathered}
{\left.\left[\sigma_{\mathrm{I}}\left(x_{0}, T, k\right)\right]\right|_{k=x_{0}} \approx \gamma_{0 a, T}+\gamma_{0 b, T} \approx \sigma_{0} \sqrt{v_{0}}} \\
\left.\partial_{k}\left[\sigma_{\mathrm{I}}\left(x_{0}, T, k\right)\right]\right|_{k=x_{0}} \approx-\gamma_{1 a, T}-\gamma_{1 b, T} \approx-\gamma_{1 a, T} \approx \frac{\sigma_{0}^{(1)} \sqrt{v_{0}}}{2}+\frac{\rho_{0} \xi_{0}}{4 \sqrt{v_{0}}} \\
\left.\partial_{k^{2}}^{2}\left[\sigma_{\mathrm{I}}\left(x_{0}, T, k\right)\right]\right|_{k=x_{0}} \approx 2 \gamma_{2, T}=\frac{\sigma_{0}^{(2)} \sqrt{v_{0}}}{3}-\frac{\left[\sigma_{0}^{(1)}\right]^{2} \sqrt{v_{0}}}{6 \sigma_{0}}-\frac{5 \rho_{0}^{2} \xi_{0}^{2}}{24 \sigma_{0} v_{0}^{\frac{3}{2}}}+\frac{\xi_{0}^{2}}{12 \sigma_{0} v_{0}^{\frac{3}{2}}},
\end{gathered}
$$

where we have used $\left|\gamma_{0 a, T}+\gamma_{0 b, T}-\bar{\sigma}\right|+\left|\gamma_{1 b, T}\right| \leq_{c} T$, and consequently neglected these terms considered as maturity bias. We have several observations.

i) In case of null correlation, our approximation coincides with [FJ11, Theorem 4.1]. The slope of ATM implied volatility depends on both the correlation and the slope of local volatility, which are therefore interpreted as skew parameters. There might be a competition between $\sigma_{0}^{(1)}$ and $\rho$ in the calibration procedures. $22]$.

ii) For pure local volatility models (i.e. $\xi_{\text {sup }}=0$ ), we retrieve the results of [BG12, Theorem

iii) For pure Heston models (i.e. $\mathcal{M}_{1}(\sigma)=0$ ), we recover the expansion given in [FJ09, Theorem 2.5]. In the case of zero correlation, the approximation formula (4.4) becomes for short maturity:

$$
\sigma_{\mathrm{I}}\left(x_{0}, T, k\right) \approx \bar{\sigma}-\frac{C_{3, T}^{s}}{16 \bar{\sigma} T}-\frac{C_{3, T}^{s}}{4 \bar{\sigma}^{3} T^{2}}+\frac{C_{3, T}^{s}}{4 \bar{\sigma}^{5} T^{3}} m^{2} \approx \bar{\sigma}-\frac{\xi_{0}^{2} \sigma_{0} T}{24 \sqrt{v_{0}}}\left[\frac{\sigma_{0}^{2} v_{0} T}{4}+1\right]+\frac{\xi_{0}^{2}}{24 \sigma_{0} v_{0}^{\frac{3}{2}}} m^{2} .
$$

We have retrieved that an uncorrelated Heston model induces symmetric smile w.r.t. the moneyness. The implied volatility is smaller ATM and becomes larger ITM or OTM, the smile increasing 
with the volatility of volatility $\xi_{0}$. If we consider a negative correlation, in view of (4.5) (the slope becomes negative and increases in absolute value with $\left|\rho_{0}\right|$ ) and (4.6) (the curvature is decreasing until reaching zero for $\left|\rho_{0}\right|=\sqrt{2 / 5} \approx 0.63$, see [FJ09, Remark 2.1]), the center of the short maturity smile is shifted to the right and the smile changes from a symmetric shape to a negative skew. The converse is realized for a positive correlation.

$\triangleright$ Calibration issues for time independent parameters. Generally the local volatility function is completely determined by the level and slope parameters identified respectively with the local volatility and its first derivative ATM. This is for instance the case of the CEV model (exposed in (5.1)). For general local and stochastic volatility models, the level of the volatility can be fixed throughout the local volatility function whereas the stochastic variance process can be normalized with an initial value $v_{0}$ equal to 1 . We have seen that for an uncorrelated local and stochastic volatility model:

i) The level parameter of the local volatility is linked to the short time implied volatility ATM,

ii) The skew parameter of the local volatility is linked to the short time slope of the implied volatility ATM,

iii) Once the local volatility function is identified, the volatility of volatility parameter is linked to the short time curvature of the implied volatility ATM.

These features allow us to suggest good surrogates for these three parameters in view of a calibration procedure by simply estimate the market implied volatility curve for short maturity. But we have observed that the correlation modifies the short term skew and it is well known that the mean reversion parameter of a CIR process is in competition with the volatility of volatility. Thus we can find models having different parameters but reproducing the same smile for one maturity or several maturities, which cause problems for calibration.

4.3. Implied volatility expansions at mid-point. It has been observed in [BG12] throughout numerical experiments that for the pure local volatility case, expansions with local volatility function frozen at mid-point $x_{a v g}=\left(x_{0}+k\right) / 2$ give better results. Actually this is a commonly admitted fact and we aim at extending this idea to the current setting. First we introduce new notations and definitions.

$\triangleright$ Corrective coefficients frozen at mid-point. The coefficients $C^{l}, C^{s}, C^{l s}$ and $\gamma$ were defined in Theorem 2.4 and Definition 4.1 for the local volatility function $\sigma$ at log-spot $x_{0}$. To consider the same coefficients but with local volatility function frozen at point $z$ (where $z$ is generally equal to $x_{a v g}$ or $\left.x_{0}\right)$, we use the notations $C_{i, T}^{l}(z), C_{i, T}^{s}(z), C_{i, T}^{l s}(z)$ and $\gamma_{i, T}(z)$.

$\triangleright$ New ellipticity assumption at $x_{\text {avg }}$. We define similarly $\left(\mathcal{H}_{x_{\text {avg }}}\right)$ and $\left(\mathcal{H}_{x_{0}}\right)$ by replacing $x_{0}$ by $x_{a v g}$ The generic constant in the further estimates will depend in an increasing way on $\frac{|\sigma|_{\infty}^{2} T}{\int_{0}^{T} \sigma_{t}^{2}\left(x_{a v g}\right) v_{t} \mathrm{~d} t}$.

$\triangleright$ Time reversal. For the coefficients $C_{i, T}^{l}\left(x_{a v g}\right)$, we introduce the notation $\widetilde{C}_{i, T}^{l}\left(x_{a v g}\right)$ which means that we have inverted the order of integration of the integrands. For example $\widetilde{C}_{1, T}^{l}\left(x_{\text {avg }}\right)=$ $\omega\left(\sigma\left(x_{\text {avg }}\right) \sigma^{(1)}\left(x_{\text {avg }}\right) v, \sigma^{2}\left(x_{\text {avg }}\right) v\right)_{0}^{T}$ instead of $C_{1, T}^{l}\left(x_{\text {avg }}\right)=\omega\left(\sigma^{2}\left(x_{\text {avg }}\right) v, \sigma\left(x_{\text {avg }}\right) \sigma^{(1)}\left(x_{\text {avg }}\right) v\right)_{0}^{T}$.

Definition 4.3. Assume $\left(\mathcal{H}_{x_{\text {avg }}}\right)$. We define the following corrective coefficients:

$\pi_{0 a, T}\left(x_{a v g}\right)=\gamma_{0 a, T}\left(x_{a v g}\right)$,

$\pi_{0 b, T}\left(x_{a v g}\right)=\gamma_{0 b, T}\left(x_{a v g}\right)$,

$\pi_{1 a, T}\left(x_{a v g}\right)=\frac{\widetilde{C}_{1, T}^{l}\left(x_{a v g}\right)-C_{1, T}^{l}\left(x_{a v g}\right)}{2 \bar{\sigma}_{x_{a v g}}^{3} T^{2}}-\frac{C_{1, T}^{s}\left(x_{a v g}\right)}{2 \bar{\sigma}_{x_{a v g}}^{3} T^{2}}$, 


$$
\begin{aligned}
\pi_{1 b, T}\left(x_{a v g}\right)= & \gamma_{1 b, T}\left(x_{a v g}\right)+\frac{C_{1, T}^{l s}\left(x_{a v g}\right)}{4 \bar{\sigma}_{x_{a v g}} T}+\frac{C_{8, T}^{l s}\left(x_{a v g}\right)}{8 \bar{\sigma}_{x_{a v g}} T}-\frac{C_{4, T}^{l s}\left(x_{a v g}\right)+C_{5, T}^{l s}\left(x_{a v g}\right)+C_{9, T}^{l s}\left(x_{a v g}\right)}{8 \bar{\sigma}_{x_{a v g}}^{3} T^{2}}, \\
\pi_{2, T}\left(x_{a v g}\right)= & \frac{\widetilde{C}_{3, T}^{l}\left(x_{a v g}\right)+C_{3, T}^{l}\left(x_{a v g}\right)}{2 \bar{\sigma}_{x_{a v g}}^{5} T^{3}}-\frac{3\left(\widetilde{C}_{4, T}^{l}\left(x_{a v g}\right)+C_{4, T}^{l}\left(x_{a v g}\right)\right)}{2 \bar{\sigma}_{x_{a v g}}^{5} T^{3}}-\frac{C_{7, T}^{l}\left(x_{a v g}\right)}{8 \bar{\sigma}_{x_{a v g}} T}+\frac{C_{8, T}^{l}\left(x_{a v g}\right)}{4 \bar{\sigma}_{x_{a v g}}^{3} T^{2}} \\
& +6 \frac{C_{6, T}^{l}\left(x_{a v g}\right)}{\bar{\sigma}_{x_{a v g}}^{7} T^{4}}+\frac{C_{2, T}^{s}\left(x_{a v g}\right)}{2 \bar{\sigma}_{x_{a v g}}^{5} T^{3}}+\frac{C_{3, T}^{s}\left(x_{a v g}\right)}{4 \bar{\sigma}_{x_{a v g}}^{5} T^{3}}-\frac{3\left(C_{1, T}^{s}\left(x_{a v g}\right)\right)^{2}}{4 \bar{\sigma}_{x_{a v g}}^{7} T^{4}}+\frac{C_{2, T}^{l s}\left(x_{a v g}\right)+C_{3, T}^{l s}\left(x_{a v g}\right)}{\bar{\sigma}_{x_{a v g}}^{5} T^{3}} \\
& +\frac{3 C_{4, T}^{l s}\left(x_{a v g}\right)}{2 \bar{\sigma}_{x_{a v g}}^{5} T^{3}}+\frac{C_{5, T}^{l s}\left(x_{a v g}\right)}{2 \bar{\sigma}_{x_{a v g}}^{5} T^{3}}+\frac{C_{6, T}^{l s}\left(x_{a v g}\right)}{2 \bar{\sigma}_{x_{a v g}}^{5} T^{3}}-\frac{3\left(C_{1, T}^{l} C_{1, T}^{s}\right)\left(x_{a v g}\right)}{\bar{\sigma}_{x_{a v g}}^{7} T^{4}}-\frac{C_{1, T}^{l s}\left(x_{a v g}\right)}{2 \bar{\sigma}_{x_{a v g}}^{3} T^{2}}-\frac{C_{8, T}^{l s}\left(x_{a v g}\right)}{4 \bar{\sigma}_{x_{a v g}}^{3} T^{2}} \\
& +3 \frac{C_{4, T}^{l s}\left(x_{a v g}\right)+C_{5, T}^{l s}\left(x_{a v g}\right)+C_{9, T}^{l s}\left(x_{a v g}\right)}{4 \bar{\sigma}_{x_{a v g}}^{5} T^{3}}
\end{aligned}
$$

where $C_{7, T}^{l}\left(x_{a v g}\right), C_{8, T}^{l}\left(x_{a v g}\right), C_{8, T}^{l s}\left(x_{a v g}\right)$ and $C_{9, T}^{l s}\left(x_{a v g}\right)$ are defined by:

$C_{7, T}^{l}\left(x_{\text {avg }}\right)=\omega\left(\left(\left(\sigma^{(1)}\right)^{2}+\sigma \sigma^{(2)}\right)\left(x_{\text {avg }}\right) v\right)_{0}^{T}, \quad C_{8, T}^{l}\left(x_{\text {avg }}\right)=\omega\left(\sigma\left(x_{\text {avg }}\right) \sigma^{(1)}\left(x_{\text {avg }}\right) v, \sigma\left(x_{\text {avg }}\right) \sigma^{(1)}\left(x_{\text {avg }}\right) v\right)_{0}^{T}$,

$C_{8, T}^{l s}\left(x_{\text {avg }}\right)=\omega\left(\rho \xi \sigma^{(1)}\left(x_{\text {avg }}\right) v, \sigma^{2}\left(x_{\text {avg }}\right)\right)_{0}^{T}, \quad C_{9, T}^{l s}\left(x_{\text {avg }}\right)=\omega\left(\sigma\left(x_{\text {avg }}\right) \sigma^{(1)}\left(x_{\text {avg }}\right) v, \rho \xi \sigma\left(x_{\text {avg }}\right) v, \sigma^{2}\left(x_{\text {avg }}\right)\right)_{0}^{T}$.

Then a tedious analysis about approximating the expansion (4.4) using $x_{\text {avg }}$ instead of $x_{0}$ leads to the following result. Details are in [Bom13].

THEOREM 4.4 (3rd order expansion of the implied volatility at mid-point). Assume $\left(\mathcal{H}_{x_{0}}\right)$, $\left(\mathcal{H}_{x_{\text {avg }}}\right)$ and $(\mathcal{P})$. We have:

$$
\begin{aligned}
\sigma_{\mathrm{I}}\left(x_{0}, T, k\right)= & \pi_{0 a, T}\left(x_{\text {avg }}\right)+\pi_{0 b, T}\left(x_{\text {avg }}\right)+\left(\pi_{1 a, T}\left(x_{\text {avg }}\right)+\pi_{1 b, T}\left(x_{\text {avg }}\right)\right) m \\
& +\pi_{2, T}\left(x_{\text {avg }}\right) m^{2}+\operatorname{Error}_{3, x_{a v g}}^{\mathrm{I}},
\end{aligned}
$$

where the corrective coefficients $\pi$ are defined in Definition 4.3, and where Error $_{3, x_{a v g}}^{\mathrm{I}}$ is estimated as in Theorem 4.2.

\section{Numerical experiments.}

5.1. Model and benchmark. Here we give numerical examples of the accuracy of our implied volatility approximation formula with local volatility at mid-point (see (4.7) in Theorem 4.4). We consider a time-independent CEV-Heston model:

$$
\begin{aligned}
\mathrm{d} S_{t} & =\mu S_{t}^{\beta} \sqrt{Y_{t}} \mathrm{~d} W_{t}, S_{0}=e^{x_{0}}, \\
\mathrm{~d} Y_{t} & =\kappa\left(\theta-Y_{t}\right) \mathrm{d} t+\sqrt{Y_{t}} \xi \mathrm{d} B_{t}, Y_{0}=v_{0}, \\
\mathrm{~d}\langle W, B\rangle_{t} & =\rho \mathrm{d} t .
\end{aligned}
$$

In our setting, it is related to local volatility function $\sigma(x)=\mu e^{(\beta-1) x}$. Using Proposition C.2 in Appendix C, the implied volatility formula (4.7) writes explicitly:

$$
\begin{aligned}
\sigma_{\mathrm{I}}\left(x_{0}, T, k\right) \approx & \mu\left(S_{0} K\right)^{\frac{\beta-1}{2}} \sqrt{\bar{v}}\left\{1+\frac{\rho \xi \mu\left(S_{0} K\right)^{\frac{\beta-1}{2}} R_{1}^{s} T}{8 \bar{v}}\right. \\
& +\frac{(\beta-1)^{2} \mu^{2}\left(S_{0} K\right)^{\beta-1} \bar{v} T}{24}\left(1-\frac{\mu^{2}\left(S_{0} K\right)^{\beta-1} \bar{v} T}{4}\right)
\end{aligned}
$$




$$
\begin{aligned}
& +\frac{\rho^{2} \xi^{2} T}{\bar{v}^{2}}\left[\frac{3\left(R_{1}^{s}\right)^{2}}{32 \bar{v}}+R_{2}^{s}\left(\frac{\mu^{2}\left(S_{0} K\right)^{\beta-1} \bar{v} T}{48}-\frac{1}{12}\right)\right]-\frac{\xi^{2} T R_{3}^{s}}{\bar{v}^{2}}\left[\frac{1}{24}+\frac{\mu^{2}\left(S_{0} K\right)^{\beta-1} \bar{v} T}{96}\right] \\
& +\frac{\rho \xi(\beta-1) \mu\left(S_{0} K\right)^{\frac{\beta-1}{2}} T}{\bar{v}}\left[\frac{R_{1}^{s}}{8}+\mu^{2}\left(S_{0} K\right)^{\beta-1} \bar{v} T\left(\frac{R_{2}^{l s}}{48 \bar{v}}-\frac{R_{1}^{s}}{32}\right)\right] \\
& +\frac{\rho \xi}{\mu\left(S_{0} K\right)^{\frac{\beta-1}{2}} \bar{v}^{2}}\left[-\frac{R_{1}^{s}}{4}+(\beta-1) \mu^{2}\left(S_{0} K\right)^{\beta-1} \bar{v} T\left(\frac{R_{1}^{s}}{16}-\frac{R_{2}^{l s}}{24 \bar{v}}\right)\right] \log \left(\frac{S_{0}}{K}\right) \\
& +\frac{\rho^{2} \xi^{2} T}{\bar{v}^{2}}\left[\frac{3\left(R_{1}^{s}\right)^{2}}{32 \bar{v}}-\frac{R_{2}^{s}}{12}\right] \log \left(\frac{S_{0}}{K}\right)-\frac{(\beta-1)^{2}}{24} \log ^{2}\left(\frac{S_{0}}{K}\right) \\
& \left.+\frac{\rho^{2} \xi^{2}}{\mu^{2}\left(S_{0} K\right)^{\beta-1} \bar{v}^{3}}\left[\frac{R_{2}^{s}}{12}-\frac{3\left(R_{1}^{s}\right)^{2}}{16 \bar{v}}\right] \log ^{2}\left(\frac{S_{0}}{K}\right)+\frac{\xi^{2} R_{3}^{s}}{24 \mu^{2}\left(S_{0} K\right)^{\beta-1} \bar{v}^{3}} \log ^{2}\left(\frac{S_{0}}{K}\right)\right\},
\end{aligned}
$$

where the coefficients $\bar{v}, R_{1}^{s}, R_{2}^{s}, R_{3}^{s}$ and $R_{2}^{l s}$ are defined in Proposition C.1. Note that if the correlation is equal to zero, many terms vanish and the formula becomes very simple.

As a benchmark, we use Monte Carlo methods with a variance reduction technique. The simulated random variable is $\left(S_{T}-K\right)_{+}$using an Euler scheme (see [Gla03, Section 3.4]) and in order to reduce the statistical error, we use the Heston control variate $\left(S_{T}^{\mathrm{H}}-K\right)_{+}-\mathbb{E}\left[\left(S_{T}^{\mathrm{H}}-K\right)_{+}\right]$ where $\left(S_{t}^{H}\right)_{t \in[0, T]}$ follows (5.1) with $\beta$ fixed at 1 . The latter expectation is computed using the Lewis formula [Lew00]. In [BGM10b], the authors have studied the numerical accuracy of price approximations w.r.t. $\kappa, \theta, \xi$ and $\rho$ in the context of Heston models whereas the influence of the parameters $\beta$ and $\mu$ has been considered in details in [BG12] in the case of pure local volatility models. This is the reason why we decide to freeze at realistic values the set of model parameters (with an important negative skew) and allow the maturity and the strike to vary in order to study the global accuracy. In all the tests we use the values:

$$
S_{0}=1, \quad \mu=0.25, \quad \beta=0.5, \quad v_{0}=1, \quad \theta=1.2, \quad \kappa=3, \quad \xi=1.5, \quad \rho=-70 \%,
$$

and we perform the Monte Carlo simulations with $10^{7}$ sample paths and a time discretization of 300 steps by year. Using the Heston control variate, this number of simulations allows to obtain confidence intervals with a width reduced to a few $\mathrm{bps}^{2}$ for a large range of strikes and maturities. All the following computations are performed using $\mathrm{C}++$ on a Intel(R) Core(TM) i5 CPU@2.40GHz with 4 GB of ram.

5.2. Accuracy of the implied volatility formula (4.7). In Tables 5.1, 5.2, 5.3, 5.4, 5.5 and 5.6 (corresponding to the maturities $6 \mathrm{M}, 1 Y, 2 Y, 3 Y, 5 Y$ and $10 Y$ ) we give for various strikes the Black-Scholes implied volatilities estimated by Monte Carlo (MC), the bounds of the 95\%confidence interval of the Monte Carlo estimator (MC- and $\mathrm{MC}+$ ) and the implied volatilities given by the approximation formula (5.2) $\left(\mathrm{AF}\left(x_{\text {avg }}\right)\right)$. We use the parameters as in (5.3) and the strikes are chosen to be approximately equal to $S_{0} e^{q \mu \sqrt{\theta T}}$ where $q$ takes the value of various quantiles of the standard Gaussian law (1\%-5\%-10\%-20\%-30\%-40\%-50\%-60\%-70\%-80\%-90\%-95\%$99 \%$ which allows to cover far ITM and far OTM options. For the sake of completeness, we report the computational time to perform the Monte Carlo simulations (for the analytical approximations, the evaluation is instantaneous).

Regarding the results, we see that our approximation formula (5.2) is very accurate, giving errors on implied volatilities smaller than $20 \mathrm{bps}$ for a large range of strikes and maturities. The results for ATM options are truly excellent but we nevertheless observe inaccuracies for extreme strikes,

\footnotetext{
${ }^{2} 1 \mathrm{bp}$ (basis point) is equal to $0.01 \%$.
} 
especially for OTM options (however for such strikes the accuracy of the Monte Carlo estimates is less good) and for short maturity. This asymmetry in the errors is probably due to the important correlation. Higher errors for short maturities is a counterintuitive fact with our error estimate (2.4). It was already observed in [BGM10b] for Heston models and it may be explained by the convergence of the stochastic variance to its stationary regime for long maturities whereas the skew is very important for short maturities due to correlation. Thus we observe a maximal error for the whole range of strikes and maturities of approximately 150 bps in Table 5.1 realized for the maturity $6 M$ and the extreme strike 1.50 . For long maturities $(3 Y, 5 Y$ and $10 Y)$, errors on implied volatility are smaller than 15 bps if we except the largest strike for which the Monte Carlo estimate is questionable (wide confidence interval). For instance we report ND in the tabulars corresponding to the maturities $5 Y$ and $10 Y$ meaning that the corresponding prices are outside the arbitrage bounds.

Last but not least, regarding the computational time, we observe that we need approximately $2 \mathrm{~m} 30 \mathrm{~s}$ per month of the maturity for the Monte Carlo simulations (4h54m27s for the maturity $10 Y$ !), whereas the whole set of implied volatilities is computed in less than $1 \mathrm{~ms}$ with the implied volatility approximation formula. This is a very significant advantage allowing real-time pricing and calibration procedures.

To conclude, our implied volatility approximation provides very good accuracy with real-time computations and it is able to handle general time-dependent local volatility functions.

TABle 5.1

Implied Black Scholes volatilities (\%) for the Monte Carlo simulations (execution time: 17m02s) and the approximation $A F\left(x_{\text {avg }}\right)$ expressed as a function of strikes for $T=6 M$.

\begin{tabular}{lrrrrrrrrrrrrr}
\hline Strikes & 0.65 & 0.75 & 0.80 & 0.85 & 0.90 & 0.95 & 1 & 1.05 & 1.10 & 1.20 & 1.25 & 1.35 & 1.50 \\
\hline $\mathrm{MC}$ & 34.86 & 31.86 & 30.49 & 29.18 & 27.94 & 26.74 & 25.61 & 24.52 & 23.50 & 21.64 & 20.82 & 19.45 & 18.01 \\
$\mathrm{MC}-$ & 34.85 & 31.86 & 30.49 & 29.18 & 27.93 & 26.74 & 25.61 & 24.52 & 23.50 & 21.64 & 20.82 & 19.44 & 17.95 \\
$\mathrm{MC}+$ & 34.87 & 31.87 & 30.49 & 29.18 & 27.94 & 26.75 & 25.61 & 24.53 & 23.50 & 21.64 & 20.83 & 19.46 & 18.07 \\
$\mathrm{AF}\left(x_{\text {avg }}\right)$ & 35.04 & 31.93 & 30.52 & 29.19 & 27.93 & 26.74 & 25.60 & 24.52 & 23.48 & 21.53 & 20.61 & 18.86 & 16.45 \\
\hline
\end{tabular}

TABle 5.2

Implied Black-Scholes volatilities (\%) for the Monte Carlo simulations (execution time: 31m33s) and the approximation $A F\left(x_{\text {avg }}\right)$ expressed as a function of strikes for $T=1 Y$.

\begin{tabular}{lrrrrrrrrrrrrr}
\hline Strikes & 0.55 & 0.65 & 0.75 & 0.80 & 0.90 & 0.95 & 1 & 1.05 & 1.15 & 1.25 & 1.40 & 1.50 & 1.80 \\
\hline $\mathrm{MC}$ & 36.36 & 33.49 & 31.01 & 29.89 & 27.85 & 26.91 & 26.02 & 25.17 & 23.61 & 22.22 & 20.43 & 19.44 & 17.32 \\
$\mathrm{MC}-$ & 36.34 & 33.48 & 31.01 & 29.89 & 27.84 & 26.90 & 26.01 & 25.17 & 23.61 & 22.22 & 20.43 & 19.43 & 17.16 \\
$\mathrm{MC}+$ & 36.37 & 33.49 & 31.02 & 29.90 & 27.85 & 26.91 & 26.02 & 25.17 & 23.62 & 22.23 & 20.44 & 19.45 & 17.47 \\
$\mathrm{AF}\left(x_{\text {avg }}\right)$ & 36.56 & 33.58 & 31.05 & 29.92 & 27.85 & 26.90 & 26.00 & 25.15 & 23.57 & 22.12 & 20.16 & 18.97 & 15.83 \\
\hline
\end{tabular}

Appendix A. Martingale property: proof of Proposition 2.3. The process $\left(e^{X_{t}}\right)_{0 \leq t \leq T}$ is a positive local martingale, thus a supermartingale and $\mathbb{E}\left[e^{X_{t}}\right] \leq e^{x_{0}}<+\infty$. The announced martingale property is proved if we show $\mathbb{E}\left[e^{X_{T}}\right]=e^{x_{0}}$.

For $n \in \mathbb{N}^{*}$, define the stopping time $\tau_{n}=\inf \left\{t \in[0, T]: V_{t} \geq n\right\}$ (with the convention $\tau_{n}=+\infty$ if the set is empty) and set

$$
\mathrm{d} X_{t}^{(n)}=\sigma\left(t, X_{t}\right) \sqrt{V_{t} \wedge n} \mathrm{~d} W_{t}-\frac{1}{2} \sigma^{2}\left(t, X_{t}\right)\left(V_{t} \wedge n\right) \mathrm{d} t, \quad X_{0}^{n}=x_{0} .
$$


TABLE 5.3

Implied Black-Scholes volatilities (\%) for the Monte Carlo simulations (execution time: 1h4m11s) and the approximation $A F\left(x_{\text {avg }}\right)$ expressed as a function of strikes for $T=2 Y$.

\begin{tabular}{|c|c|c|c|c|c|c|c|c|c|c|c|c|c|}
\hline Strikes & 0.45 & 0.55 & 0.65 & 0.75 & 0.85 & 0.90 & 1 & 1.10 & 1.20 & 1.35 & 1.55 & 1.80 & 2.30 \\
\hline $\mathrm{MC}$ & 37.22 & 34.46 & 32.19 & 30.26 & 28.59 & 27.83 & 26.44 & 25.20 & 24.07 & 22.58 & 20.89 & 19.16 & 16.72 \\
\hline MC- & 37.20 & 34.45 & 32.18 & 30.26 & 28.59 & 27.83 & 26.44 & 25.19 & 24.07 & 22.58 & 20.88 & 19.14 & 16.26 \\
\hline $\mathrm{MC}+$ & 37.24 & 34.47 & 32.20 & 30.27 & 28.60 & 27.84 & 26.45 & 25.20 & 24.08 & 22.59 & 20.89 & 19.18 & 17.05 \\
\hline $\operatorname{AF}\left(x_{a v g}\right)$ & 37.32 & 34.52 & 32.22 & 30.28 & 28.59 & 27.83 & 26.43 & 25.18 & 24.04 & 22.52 & 20.76 & 18.87 & 15.84 \\
\hline
\end{tabular}

TABLE 5.4

Implied Black-Scholes volatilities (\%) for the Monte Carlo simulations (execution time: 1h31m44s) and the approximation $A F\left(x_{\text {avg }}\right)$ expressed as a function of strikes for $T=3 Y$.

\begin{tabular}{|c|c|c|c|c|c|c|c|c|c|c|c|c|c|}
\hline Strikes & 0.35 & 0.50 & 0.55 & 0.70 & 0.80 & 0.90 & 1 & 1.10 & 1.25 & 1.45 & 1.75 & 2.05 & 2.70 \\
\hline $\mathrm{MC}$ & 39.08 & 34.73 & 33.59 & 30.74 & 29.19 & 27.84 & 26.65 & 25.57 & 24.16 & 22.55 & 20.57 & 18.97 & 16.32 \\
\hline MC- & 39.04 & 34.71 & 33.58 & 30.74 & 29.19 & 27.84 & 26.64 & 25.57 & 24.15 & 22.54 & 20.55 & 18.94 & 15.58 \\
\hline $\mathrm{MC}+$ & 39.11 & 34.74 & 33.60 & 30.75 & 29.20 & 27.85 & 26.65 & 25.58 & 24.16 & 22.55 & 20.58 & 19.00 & 16.79 \\
\hline $\operatorname{AF}\left(x_{a v g}\right)$ & 39.13 & 34.76 & 33.62 & 30.76 & 29.20 & 27.85 & 26.65 & 25.58 & 24.15 & 22.52 & 20.49 & 18.82 & 15.97 \\
\hline
\end{tabular}

By the monotone convergence theorem and the local property of stochastic integrals, we have

$$
\mathbb{E}\left[e^{X_{T}}\right]=\lim _{n \rightarrow+\infty} \mathbb{E}\left[e^{X_{T}} \mathbb{1}_{T<\tau_{n}}\right]=\lim _{n \rightarrow+\infty} \mathbb{E}\left[e^{X_{T}^{(n)}} \mathbb{1}_{T<\tau_{n}}\right] .
$$

Because $\sigma\left(t, X_{t}\right) \sqrt{V_{t} \wedge n}$ is bounded in $(t, \omega)$, we can define a new measure $\left.\mathbb{Q}^{(n)}\right|_{\mathcal{F}_{T}}=\left.e^{X_{T}^{(n)}-x_{0}} \mathbb{Q}\right|_{\mathcal{F}_{T}}$ under which

$$
\left(W_{t}^{(n)}, B_{t}^{(n)}\right):=\left(W_{t}-\int_{0}^{t} \sigma\left(s, X_{s}\right) \sqrt{V_{s} \wedge n} \mathrm{~d} s, B_{t}-\int_{0}^{t} \rho_{s} \sigma\left(s, X_{s}\right) \sqrt{V_{s} \wedge n} \mathrm{~d} s\right)
$$

is a two-dimensional Brownian motion with correlation $\left(\rho_{t}\right)_{0 \leq t \leq T}$. It gives

$$
\mathbb{E}\left[e^{X_{T}}\right]=e^{x_{0}} \lim _{n \rightarrow+\infty} \mathbb{Q}^{(n)}\left(T<\tau_{n}\right) .
$$

The dynamics of $V$ under $\mathbb{Q}^{(n)}$ writes

$$
\mathrm{d} V_{t}=\left(\alpha_{t}+\rho_{t} \xi_{t} \sigma\left(t, X_{t}\right) \sqrt{V_{t}} \sqrt{V_{t} \wedge n}\right) \mathrm{d} t+\xi_{t} \sqrt{V_{t}} \mathrm{~d} B_{t}^{(n)}, \quad V_{0}=v_{0},
$$

and we aim at comparing it to the Stochastic Differential Equation (SDE in short)

$$
\mathrm{d} V_{t}^{(n)}=\left(\alpha_{t}+|\xi|_{\infty}|\sigma|_{\infty} V_{t}^{(n)}\right) \mathrm{d} t+\xi_{t} \sqrt{V_{t}^{(n)}} \mathrm{d} B_{t}^{(n)}, \quad V_{0}^{(n)}=v_{0} .
$$

Observe that the drift coefficient of $V^{(n)}$ dominates that of $V$ : therefore, from usual arguments used for comparing time-dependent SDEs (see Proposition [KS91, Proposition 5.2.18]), we can show $V_{t}^{(n)} \geq V_{t}$ for any $t \in[0, T]$ a.s.. Actually, the quoted reference deals with one-dimensional SDEs, but the arguments apply in the same way to our model $(X, V)$. Then it gives

$$
\mathbb{Q}^{(n)}\left(T<\tau_{n}\right) \geq \mathbb{Q}^{(n)}\left(\sup _{0 \leq t \leq T} V_{t}^{(n)}<n\right) .
$$


TABLE 5.5

Implied Black-Scholes volatilities (\%) for the Monte Carlo simulations (execution time: 2h29m18s) and the approximation $A F\left(x_{\text {avg }}\right)$ expressed as a function of strikes for $T=5 Y$.

\begin{tabular}{lrrrrrrrrrrrrl}
\hline Strikes & 0.25 & 0.40 & 0.50 & 0.60 & 0.75 & 0.85 & 1 & 1.15 & 1.35 & 1.60 & 2.05 & 2.50 & 3.60 \\
\hline $\mathrm{MC}$ & 41.27 & 36.15 & 33.81 & 31.93 & 29.68 & 28.44 & 26.86 & 25.52 & 24.01 & 22.45 & 20.25 & 18.53 & 15.59 \\
$\mathrm{MC}-$ & 41.21 & 36.12 & 33.79 & 31.91 & 29.67 & 28.43 & 26.85 & 25.51 & 24.01 & 22.45 & 20.23 & 18.48 & ND \\
$\mathrm{MC}+$ & 41.33 & 36.18 & 33.82 & 31.94 & 29.69 & 28.45 & 26.86 & 25.53 & 24.02 & 22.46 & 20.27 & 18.57 & 16.76 \\
$\mathrm{AF}\left(x_{\text {avg }}\right)$ & 41.27 & 36.16 & 33.82 & 31.94 & 29.69 & 28.45 & 26.87 & 25.53 & 24.03 & 22.46 & 20.24 & 18.51 & 15.44 \\
\hline
\end{tabular}

TABLe 5.6

Implied Black-Scholes volatilities (\%) for the Monte Carlo simulations (execution time: 4h54m27s) and the approximation $A F\left(x_{a v g}\right)$ expressed as a function of strikes for $T=10 Y$.

\begin{tabular}{lrrrrrrrrrrrrr}
\hline Strikes & 0.15 & 0.25 & 0.35 & 0.50 & 0.65 & 0.80 & 1 & 1.20 & 1.50 & 1.95 & 2.75 & 3.65 & 6.30 \\
\hline MC & 44.71 & 39.62 & 36.40 & 33.11 & 30.77 & 28.97 & 27.09 & 25.59 & 23.81 & 21.79 & 19.28 & 17.33 & 15.31 \\
MC- & 44.60 & 39.56 & 36.36 & 33.08 & 30.75 & 28.95 & 27.07 & 25.58 & 23.80 & 21.78 & 19.25 & 17.22 & ND \\
MC+ & 44.83 & 39.69 & 36.44 & 33.14 & 30.79 & 28.98 & 27.10 & 25.60 & 23.82 & 21.81 & 19.31 & 17.43 & 16.28 \\
$\mathrm{AF}\left(x_{\text {avg }}\right)$ & 44.69 & 39.63 & 36.41 & 33.12 & 30.78 & 28.98 & 27.10 & 25.61 & 23.83 & 21.81 & 19.28 & 17.30 & 13.70
\end{tabular}

The last probability is equal to $\mathbb{Q}\left(\sup _{0 \leq t \leq T} \tilde{V}_{t}<n\right)$ where $\tilde{V}$ is the (strong) solution to (A.3) with $B$ instead of $B^{(n)}$. Since $\tilde{V}$ is non explosive, the latter probability converges to 1 as $n \rightarrow+\infty$. The proof of Proposition 2.3 is complete.

Appendix B. Explicit computation of the corrective terms of Theorem 2.4. We give the full derivation of the corrective terms in the approximation (2.3) of Theorem 2.4. We begin with the proof of Lemma 3.2 and next we give the details of the computation of the corrective terms.

B.1. Proof of Lemma 3.2. We proceed by induction, by using the next intermediate result:

Lemma B.1. Let $\left(M_{t}\right)_{t \in[0, T]}$ be a square integrable and predictable process, $\left(f_{t}\right)_{t \in[0, T]}$ be a measurable and bounded deterministic function and $\varphi \in \mathcal{C}_{b}^{\infty}(\mathbb{R})$. Then, we have:

$$
\begin{aligned}
& \mathbb{E}\left[\varphi\left(\int_{0}^{T} f_{t} \mathrm{~d} W_{t}\right) \int_{0}^{T} M_{t} \mathrm{~d} W_{t}\right]=\mathbb{E}\left[\varphi^{(1)}\left(\int_{0}^{T} f_{t} \mathrm{~d} W_{t}\right) \int_{0}^{T} f_{t} M_{t} \mathrm{~d} t\right], \\
& \mathbb{E}\left[\varphi\left(\int_{0}^{T} f_{t} \mathrm{~d} W_{t}\right) \int_{0}^{T} M_{t} \mathrm{~d} B_{t}\right]=\mathbb{E}\left[\varphi^{(1)}\left(\int_{0}^{T} f_{t} \mathrm{~d} W_{t}\right) \int_{0}^{T} \rho_{t} f_{t} M_{t} \mathrm{~d} t\right] .
\end{aligned}
$$

Proof. These equalities directly follow from the duality relationship of Malliavin calculus (see Lemma 1.2.1 in [Nua06]).

If $N=1$ and $I_{N}=0$, there is nothing to prove. If $N=1$ and $I_{N} \in\{1,2\}$, Lemma 3.2 is a particular case of Lemma B.1 (with deterministic $M$ ) noting that $\forall i \in \mathbb{N}$,

$$
\mathbb{E}\left[\varphi^{(i)}\left(\int_{0}^{T} f_{t} \mathrm{~d} W_{t}\right)\right]=\left.\partial_{x^{i}}^{i} \mathbb{E}\left[\varphi\left(\int_{0}^{T} f_{t} \mathrm{~d} W_{t}+x\right)\right]\right|_{x=0} .
$$

Suppose that the formula (3.13) is true for a given $N \geq 1$ and let us prove it at order $N+1$. Then 
write, directly if $I_{N+1}=0$ or with Lemma B.1 if $I_{N+1} \in\{1,2\}$,

$$
\begin{aligned}
& \mathbb{E}\left[\varphi\left(\int_{0}^{T} f_{t} \mathrm{~d} W_{t}\right) \int_{0}^{T} l_{N+1, t_{N+1}} \int_{0}^{t_{N+1}} l_{N, t_{N}} \ldots \int_{0}^{t_{2}} l_{1, t_{1}} \mathrm{~d} W_{t_{1}}^{I_{1}} \ldots \mathrm{d} W_{t_{N}}^{I_{N}} \mathrm{~d} W_{t_{N+1}}^{I_{N+1}}\right] \\
= & \mathbb{E}\left[\varphi^{\left(\mathbb{1}_{I_{N+1} \neq 0}\right)}\left(\int_{0}^{T} f_{t} \mathrm{~d} W_{t}\right) \int_{0}^{T} \widehat{l}_{N+1, t_{N+1}} \int_{0}^{t_{N+1}} l_{N, t_{N}} \int_{0}^{t_{N}} \ldots \int_{0}^{t_{2}} l_{1, t_{1}} \mathrm{~d} W_{t_{1}}^{I_{1}} \ldots \mathrm{d} W_{t_{N}}^{I_{N}} \mathrm{~d} t_{N+1}\right] \\
= & \mathbb{E}\left[\varphi^{\left(\mathbb{1}_{I_{N+1} \neq 0}\right)}\left(\int_{0}^{T} f_{t} \mathrm{~d} W_{t}\right) \int_{0}^{T}\left(l_{N, t_{N}} \int_{t_{N}}^{T} \widehat{l}_{N+1, s} \mathrm{~d} s\right) \int_{0}^{t_{N}} \ldots \int_{0}^{t_{2}} l_{1, t_{1}} \mathrm{~d} W_{t_{1}}^{I_{1}} \ldots \mathrm{d} W_{t_{N}}^{I_{N}}\right],
\end{aligned}
$$

where at the last equality we have used the Itô integration by parts formula $\int_{0}^{T} g_{t} Z_{t} \mathrm{~d} t=\int_{0}^{T}\left(\int_{t}^{T} g_{s} \mathrm{~d} s\right) \mathrm{d} Z_{t}$, valid for any continuous semi-martingale $Z$ starting from 0 and any measurable and bounded deterministic function $g$. We easily conclude using the induction hypothesis at order $N$.

B.2. Calculus of the corrective terms. We recall our third order approximation:

$$
\mathbb{E}\left[h\left(X_{T}^{P}\right)\right]+\mathbb{E}\left[h^{(1)}\left(X_{T}^{P}\right) X_{1, T}\right]+\mathbb{E}\left[h^{(1)}\left(X_{T}^{P}\right) \frac{X_{2, T}}{2}\right]+\frac{1}{2} \mathbb{E}\left[h^{(2)}\left(X_{T}^{P}\right) X_{1, T}^{2}\right] .
$$

We compute each corrective term separately, and pay attention to the different natures in these corrections (pure local volatility, pure stochastic volatility and mixed local-stochastic one).

$\triangleright$ Step 1: contribution with $X_{1, T}$. From (3.4)-(3.5)-(3.6) we have

$$
X_{1, T}=\int_{0}^{T}\left(\sigma_{t_{2}}^{(1)} \lambda_{t_{2}} \int_{0}^{t_{2}} \sigma_{t_{1}} \lambda_{t_{1}}\left(\mathrm{~d} W_{t_{1}}-\frac{\sigma_{t_{1}} \lambda_{t_{1}}}{2} \mathrm{~d} t_{1}\right)+\frac{\sigma_{t_{2}}}{2 \lambda_{t_{2}}} \int_{0}^{t_{2}} \xi_{t_{1}} \lambda_{t_{1}} \mathrm{~d} B_{t_{1}}\right)\left(\mathrm{d} W_{t_{2}}-\sigma_{t_{2}} \lambda_{t_{2}} \mathrm{~d} t_{2}\right) .
$$

Then, apply repeatedly Lemma 3.2 to $\varphi(\cdot)=h^{(1)}\left(x_{0}-\frac{1}{2} \int_{0}^{T} \sigma_{t}^{2} v_{t} \mathrm{~d} t+\cdot\right), f_{t}=\sigma_{t} \lambda_{t}$ to get (recalling the definition (2.1) of sensitivities):

$$
\mathbb{E}\left[h^{(1)}\left(X_{T}^{P}\right) X_{1, T}\right]=C_{1, T}^{l}\left[\mathcal{G}_{3}^{h}-\frac{3}{2} \mathcal{G}_{2}^{h}+\frac{1}{2} \mathcal{G}_{1}^{h}\right]+\frac{C_{1, T}^{s}}{2}\left[\mathcal{G}_{3}^{h}-\mathcal{G}_{2}^{h}\right],
$$

where:

$$
C_{1, T}^{l}=\omega\left(\sigma^{2} v, \sigma \sigma^{(1)} v\right)_{0}^{T}, \quad C_{1, T}^{s}=\omega\left(\rho \xi \sigma v, \sigma^{2}\right)_{0}^{T} .
$$

$\triangleright$ Step 2: contribution with $X_{2, T}$. In view of (3.7) and (3.6)-(3.9), we have:

$$
\begin{aligned}
\frac{X_{2, T}}{2}= & \frac{1}{2} \int_{0}^{T} \lambda_{t}\left[\left(X_{t}^{P}-x_{0}\right)^{2} \sigma_{t}^{(2)}+2 X_{1, t} \sigma_{t}^{(1)}\right]\left(\mathrm{d} W_{t}-\sigma_{t} \lambda_{t} \mathrm{~d} t\right) \\
& +\frac{1}{2} \int_{0}^{T}\left(X_{t}^{P}-x_{0}\right) V_{1, t} \frac{\sigma_{t}^{(1)}}{\lambda_{t}}\left(\mathrm{~d} W_{t}-2 \sigma_{t} \lambda_{t} \mathrm{~d} t\right) \\
& -\frac{1}{2} \int_{0}^{T}\left(X_{t}^{P}-x_{0}\right)^{2}\left(\sigma_{t}^{(1)}\right)^{2} v_{t} \mathrm{~d} t+\frac{1}{4} \int_{0}^{T} \frac{V_{2, t}}{\lambda_{t}} \sigma_{t}\left(\mathrm{~d} W_{t}-\sigma_{t} \lambda_{t} \mathrm{~d} t\right)-\frac{1}{8} \int_{0}^{T} \frac{V_{1, t}^{2}}{\lambda_{t}^{3}} \sigma_{t} \mathrm{~d} W_{t} .
\end{aligned}
$$

Besides, the Itô formula combined with (1.4)-(3.4)-(3.5)-(3.6)-(3.9) yields

$$
\frac{\left(X_{t}^{P}-x_{0}\right)^{2}}{2}=\int_{0}^{t}\left(\int_{0}^{t_{2}} \sigma_{t_{1}} \lambda_{t_{1}}\left(\mathrm{~d} W_{t_{1}}-\frac{\sigma_{t_{1}} \lambda_{t_{1}}}{2} \mathrm{~d} t_{1}\right)\right) \sigma_{t_{2}} \lambda_{t_{2}}\left(\mathrm{~d} W_{t_{2}}-\frac{\sigma_{t_{2}} \lambda_{t_{2}}}{2} \mathrm{~d} t_{2}\right)
$$




$$
\begin{aligned}
& +\frac{1}{2} \int_{0}^{t} \sigma_{t_{1}}^{2} v_{t_{1}} \mathrm{~d} t_{1} \\
X_{1, t}= & \int_{0}^{t}\left(\int_{0}^{t_{2}} \sigma_{t_{1}} \lambda_{t_{1}}\left(\mathrm{~d} W_{t_{1}}-\frac{\sigma_{t_{1}} \lambda_{t_{1}}}{2} \mathrm{~d} t_{1}\right)\right) \sigma_{t_{2}}^{(1)} \lambda_{t_{2}}\left(\mathrm{~d} W_{t_{2}}-\sigma_{t_{2}} \lambda_{t_{2}} \mathrm{~d} t_{2}\right) \\
& +\int_{0}^{t} \frac{\sigma_{t_{2}}}{2 \lambda_{t_{2}}}\left(\int_{0}^{t_{2}} \xi_{t_{1}} \lambda_{t_{1}} \mathrm{~d} B_{t_{1}}\right)\left(\mathrm{d} W_{t_{2}}-\sigma_{t_{2}} \lambda_{t_{2}} \mathrm{~d} t_{2}\right) \\
\left(X_{t}^{P}-x_{0}\right) V_{1, t}= & \int_{0}^{t}\left(\int_{0}^{t_{2}} \xi_{s_{1}} \lambda_{s_{1}} \mathrm{~d} B_{s_{1}}\right) \sigma_{t_{2}} \lambda_{t_{2}}\left(\mathrm{~d} W_{t_{2}}-\frac{\sigma_{t_{2}} \lambda_{t_{2}}}{2} \mathrm{~d} t_{2}\right) \\
& +\int_{0}^{t}\left(\int_{0}^{t_{2}} \sigma_{t_{1}} \lambda_{t_{1}}\left(\mathrm{~d} W_{t_{1}}-\frac{\sigma_{t_{1}} \lambda_{t_{1}}}{2} \mathrm{~d} t_{1}\right)\right) \xi_{s_{2}} \lambda_{s_{2}} \mathrm{~d} B_{s_{2}}+\int_{0}^{t} \xi_{t_{1}} \rho_{t_{1}} \sigma_{t_{1}} v_{t_{1}} \mathrm{~d} t_{1}, \\
V_{2, t}= & \int_{0}^{t}\left(\int_{0}^{t_{2}} \xi_{s_{1}} \lambda_{s_{1}} \mathrm{~d} B_{s_{1}}\right) \frac{\xi_{s_{2}}}{\lambda_{s_{2}}} \mathrm{~d} B_{s_{2}}
\end{aligned}
$$

Combining (B.1)-(B.3)-(B.4)-(B.5)-(B.6) with repeated applications of Lemma 3.2 yields (after tedious but easy computations)

$$
\begin{aligned}
& \mathbb{E}\left[h^{(1)}\left(X_{T}^{P}\right) \frac{X_{2, T}}{2}\right]+\frac{1}{8} \mathbb{E}\left[h^{(2)}\left(X_{T}^{P}\right) \int_{0}^{T} \frac{V_{1, t}^{2}}{v_{t}} \sigma_{t}^{2} \mathrm{~d} t\right] \\
= & C_{3 b, T}^{l}\left[\mathcal{G}_{4}^{h}-2 \mathcal{G}_{3}^{h}+\frac{5 \mathcal{G}_{2}^{h}}{4}-\frac{\mathcal{G}_{1}^{h}}{4}\right]+C_{2 b, T}^{l}\left[\frac{\mathcal{G}_{2}^{h}}{2}-\frac{\mathcal{G}_{1}^{h}}{2}\right] \\
& +C_{4, T}^{l}\left[\mathcal{G}_{4}^{h}-\frac{5 \mathcal{G}_{3}^{h}}{2}+2 \mathcal{G}_{2}^{h}-\frac{\mathcal{G}_{1}^{h}}{2}\right]+C_{4, T}^{l s}\left[\frac{\mathcal{G}_{4}^{h}}{2}-\mathcal{G}_{3}^{h}+\frac{\mathcal{G}_{2}^{h}}{2}\right] \\
& +\left(C_{2, T}^{l s}+C_{3, T}^{l s}\right)\left[\frac{\mathcal{G}_{4}^{h}}{2}-\frac{5 \mathcal{G}_{3}^{h}}{4}+\frac{\mathcal{G}_{2}^{h}}{2}\right]+C_{1, T}^{l s}\left[\frac{\mathcal{G}_{2}^{h}}{2}-\mathcal{G}_{1}^{h}\right] \\
& +C_{3 a, T}^{l}\left[-\mathcal{G}_{3}^{h}+\mathcal{G}_{2}^{h}-\frac{\mathcal{G}_{1}^{h}}{4}\right]-C_{2 a, T}^{l} \frac{\mathcal{G}_{1}^{h}}{2}+C_{2, T}^{s}\left[\frac{\mathcal{G}_{4}^{h}}{4}-\frac{\mathcal{G}_{3}^{h}}{4}\right],
\end{aligned}
$$

where:

$$
\begin{aligned}
& C_{2 a, T}^{l}=\omega\left(\sigma^{2} v,\left(\sigma^{(1)}\right)^{2} v\right)_{0}^{T}, \quad C_{2 b, T}^{l}=\omega\left(\sigma^{2} v, \sigma \sigma^{(2)} v\right)_{0}^{T}, \quad C_{3 a, T}^{l}=\omega\left(\sigma^{2} v, \sigma^{2} v,\left(\sigma^{(1)}\right)^{2} v\right)_{0}^{T}, \\
& C_{3 b, T}^{l}=\omega\left(\sigma^{2} v, \sigma^{2} v, \sigma \sigma^{(2)} v\right)_{0}^{T}, \quad C_{4, T}^{l}=\omega\left(\sigma^{2} v, \sigma \sigma^{(1)} v, \sigma \sigma^{(1)} v\right)_{0}^{T}, \quad C_{2, T}^{s}=\omega\left(\rho \xi \sigma v, \rho \xi \sigma, \sigma^{2}\right)_{0}^{T} \text {, } \\
& C_{1, T}^{l s}=\omega\left(\rho \xi \sigma v, \sigma \sigma^{(1)}\right)_{0}^{T}, \quad C_{2, T}^{l s}=\omega\left(\rho \xi \sigma v, \sigma^{2} v, \sigma \sigma^{(1)}\right)_{0}^{T}, \quad C_{3, T}^{l s}=\omega\left(\sigma^{2} v, \rho \xi \sigma v, \sigma \sigma^{(1)}\right)_{0}^{T} \text {, } \\
& C_{4, T}^{l s}=\omega\left(\rho \xi \sigma v, \sigma^{2}, \sigma \sigma^{(1)} v\right)_{0}^{T} \text {. }
\end{aligned}
$$

Here we handle the second term of (B.7) together with $X_{2, T}$ because the overall computations are somewhat simpler (here it has the effect of removing the last contribution of $X_{2, T}$ ).

$\triangleright$ Step 3: contribution with $X_{1, T}^{2}$. Starting from (3.4) and applying the Itô formula we have:

$$
\begin{aligned}
\frac{1}{2} X_{1, T}^{2}= & \int_{0}^{T} X_{1, t}\left(\left(X_{t}^{P}-x_{0}\right) \sigma_{t}^{(1)} \lambda_{t}+\frac{V_{1, t} \sigma_{t}}{2 \lambda_{t}}\right)\left(\mathrm{d} W_{t}-\sigma_{t} \lambda_{t} \mathrm{~d} t\right) \\
& +\frac{1}{2} \int_{0}^{T}\left(\left(X_{t}^{P}-x_{0}\right)^{2}\left(\sigma_{t}^{(1)}\right)^{2} v_{t}+\left(X_{t}^{P}-x_{0}\right) V_{1, t} \sigma_{t} \sigma_{t}^{(1)}\right) \mathrm{d} t+\frac{1}{8} \int_{0}^{T} \frac{V_{1, t}^{2}}{v_{t}} \sigma_{t}^{2} \mathrm{~d} t, \\
X_{1, t}\left(X_{t}^{P}-x_{0}\right)= & \int_{0}^{t}\left[\left(X_{t_{1}}^{P}-x_{0}\right)^{2} \sigma_{t_{1}}^{(1)} \lambda_{t_{1}}+\frac{\left(X_{t_{1}}^{P}-x_{0}\right) V_{1, t_{1}} \sigma_{t_{1}}}{2 \lambda_{t_{1}}}\right]\left(\mathrm{d} W_{t_{1}}-\sigma_{t_{1}} \lambda_{t_{1}} \mathrm{~d} t_{1}\right)
\end{aligned}
$$




$$
\begin{aligned}
& +\int_{0}^{t} X_{1, t_{1}} \sigma_{t_{1}} \lambda_{t_{1}}\left(\mathrm{~d} W_{t_{1}}-\frac{\sigma_{t_{1}} \lambda_{t_{1}}}{2} \mathrm{~d} t_{1}\right)+\int_{0}^{t}\left[\left(X_{t_{1}}^{P}-x_{0}\right) \sigma_{t_{1}} \sigma_{t_{1}}^{(1)} v_{t_{1}}+\frac{V_{1, t_{1}}}{2} \sigma_{t_{1}}^{2}\right] \mathrm{d} t_{1}, \\
X_{1, t} V_{1, t}= & \int_{0}^{T}\left[\left(X_{t_{1}}^{P}-x_{0}\right) V_{1, t_{1}} \sigma_{t_{1}}^{(1)} \lambda_{t_{1}}+\frac{V_{1, t_{1}}^{2} \sigma_{t_{1}}}{2 \lambda_{t_{1}}}\right]\left(\mathrm{d} W_{t_{1}}-\sigma_{t_{1}} \lambda_{t_{1}} \mathrm{~d} t_{1}\right)+\int_{0}^{t} X_{1, t_{1}} \xi_{t_{1}} \lambda_{t_{1}} \mathrm{~d} B_{t_{1}} \\
& +\int_{0}^{t} \rho_{t_{1}} \xi_{t_{1}}\left[\left(X_{t_{1}}^{P}-x_{0}\right) \sigma_{t_{1}}^{(1)} v_{t_{1}}+\frac{V_{1, t_{1}} \sigma_{t_{1}}}{2}\right] \mathrm{d} t_{1}, \\
V_{1, t}^{2}= & 2 \int_{0}^{t}\left(\int_{0}^{t_{2}} \xi_{t_{1}} \lambda_{t_{1}} \mathrm{~d} B_{t_{1}}\right) \xi_{t_{2}} \lambda_{t_{2}} \mathrm{~d} B_{t_{2}}+\int_{0}^{t} \xi_{t_{1}}^{2} v_{t_{1}} \mathrm{~d} t_{1} .
\end{aligned}
$$

From Lemma 3.2 and (B.9)-(B.3)-(B.5)-(B.1)-(1.4)-(3.5) it follows that

$$
\begin{aligned}
& \mathbb{E}\left[h^{(2)}\left(X_{T}^{P}\right) \int_{0}^{T} X_{1, t}\left(X_{t}^{P}-x_{0}\right) \sigma_{t}^{(1)} \lambda_{t}\left(\mathrm{~d} W_{t}-\sigma_{t} \lambda_{t} \mathrm{~d} t\right)\right] \\
= & C_{9 a, T}^{l}\left[2 \mathcal{G}_{6}^{h}-6 \mathcal{G}_{5}^{h}+\frac{13 \mathcal{G}_{4}^{h}}{2}-3 \mathcal{G}_{3}^{h}+\frac{\mathcal{G}_{2}^{h}}{2}\right]+C_{4, T}^{l}\left[\mathcal{G}_{4}^{h}-2 \mathcal{G}_{3}^{h}+\mathcal{G}_{2}^{h}\right] \\
& +\left(C_{10 a, T}^{l s}+C_{10 b, T}^{l s}\right)\left[\frac{\mathcal{G}_{6}^{h}}{2}-\frac{5 \mathcal{G}_{5}^{h}}{4}+\mathcal{G}_{4}^{h}-\frac{\mathcal{G}_{3}^{h}}{4}\right]+C_{4, T}^{l s}\left[\frac{\mathcal{G}_{4}^{h}}{2}-\mathcal{G}_{3}^{h}+\frac{\mathcal{G}_{2}^{h}}{2}\right] \\
& +C_{9 b, T}^{l}\left[\mathcal{G}_{6}^{h}-3 \mathcal{G}_{5}^{h}+\frac{13 \mathcal{G}_{4}^{h}}{4}-\frac{3 \mathcal{G}_{3}^{h}}{2}+\frac{\mathcal{G}_{2}^{h}}{4}\right]+C_{10 c, T}^{l s}\left[\frac{\mathcal{G}_{6}^{h}}{2}-\frac{5 \mathcal{G}_{5}^{h}}{4}+\mathcal{G}_{4}^{h}-\frac{\mathcal{G}_{3}^{h}}{4}\right] \\
& +C_{4, T}^{l}\left[\mathcal{G}_{4}^{h}-\frac{3 \mathcal{G}_{3}^{h}}{2}+\frac{\mathcal{G}_{2}^{h}}{2}\right]+C_{4, T}^{l s}\left[\frac{\mathcal{G}_{4}^{h}}{2}-\frac{\mathcal{G}_{3}^{h}}{2}\right]
\end{aligned}
$$

where

$$
\begin{aligned}
C_{9 a, T}^{l} & =\omega\left(\sigma^{2} v, \sigma^{2} v, \sigma \sigma^{(1)} v, \sigma \sigma^{(1)} v\right)_{0}^{T}, & & C_{9 b, T}^{l}=\omega\left(\sigma^{2} v, \sigma \sigma^{(1)} v, \sigma^{2} v, \sigma \sigma^{(1)} v\right)_{0}^{T}, \\
C_{10 a, T}^{l s} & =\omega\left(\rho \xi \sigma v, \sigma^{2} v, \sigma^{2}, \sigma \sigma^{(1)} v\right)_{0}^{T}, & & C_{10 b, T}^{l s}=\omega\left(\sigma^{2} v, \rho \xi \sigma v, \sigma^{2}, \sigma \sigma^{(1)} v\right)_{0}^{T}, \\
C_{10 c, T}^{l s} & =\omega\left(\rho \xi \sigma v, \sigma^{2}, \sigma^{2} v, \sigma \sigma^{(1)} v\right)_{0}^{T} . & &
\end{aligned}
$$

Similarly, using Lemma 3.2 and (B.10)-(B.5)-(B.11)-(B.1), we have:

$$
\begin{aligned}
& \frac{1}{2} \mathbb{E}\left[h^{(2)}\left(X_{T}^{P}\right) \int_{0}^{T} \frac{X_{1, t} V_{1, t} \sigma_{t}}{\lambda_{t}}\left(\mathrm{~d} W_{t}-\sigma_{t} \lambda_{t} \mathrm{~d} t\right)\right] \\
= & \left(C_{10 d, T}^{l s}+C_{10 e, T}^{l s}\right)\left[\frac{\mathcal{G}_{6}^{h}}{2}-\frac{5 \mathcal{G}_{5}^{h}}{4}+\mathcal{G}_{4}^{h}-\frac{\mathcal{G}_{3}^{h}}{4}\right]+C_{5, T}^{l s}\left[\frac{\mathcal{G}_{4}^{h}}{2}-\mathcal{G}_{3}^{h}+\frac{\mathcal{G}_{2}^{h}}{2}\right] \\
& +C_{4 a, T}^{s}\left[\frac{\mathcal{G}_{6}^{h}}{2}-\mathcal{G}_{5}^{h}+\frac{\mathcal{G}_{4}^{h}}{2}\right]+C_{3, T}^{s}\left[\frac{\mathcal{G}_{4}^{h}}{4}-\frac{\mathcal{G}_{3}^{h}}{2}+\frac{\mathcal{G}_{2}^{h}}{4}\right] \\
& +C_{10 f, T}^{l s}\left[\frac{\mathcal{G}_{6}^{h}}{2}-\frac{5 \mathcal{G}_{5}^{h}}{4}+\mathcal{G}_{4}^{h}-\frac{\mathcal{G}_{3}^{h}}{4}\right]+C_{4 b, T}^{s}\left[\frac{\mathcal{G}_{6}^{h}}{4}-\frac{\mathcal{G}_{5}^{h}}{2}+\frac{\mathcal{G}_{4}^{h}}{4}\right] \\
& +C_{6, T}^{l s}\left[\frac{\mathcal{G}_{4}^{h}}{2}-\frac{3 \mathcal{G}_{3}^{h}}{4}+\frac{\mathcal{G}_{2}^{h}}{4}\right]+C_{2, T}^{s}\left[\frac{\mathcal{G}_{4}^{h}}{4}-\frac{\mathcal{G}_{3}^{h}}{4}\right],
\end{aligned}
$$

where

$$
\begin{aligned}
& C_{2, T}^{s}=\omega\left(\rho \xi \sigma v, \rho \xi \sigma, \sigma^{2}\right)_{0}^{T}, \quad C_{3, T}^{s}=\omega\left(\xi^{2} v, \sigma^{2}, \sigma^{2}\right)_{0}^{T}, \quad C_{4 a, T}^{s}=\omega\left(\rho \xi \sigma v, \xi \rho v \sigma, \sigma^{2}, \sigma^{2}\right)_{0}^{T}, \\
& C_{4 b, T}^{s}=\omega\left(\rho \xi v \sigma, \sigma^{2}, \rho \xi \sigma v, \sigma^{2}\right)_{0}^{T}, \quad C_{5, T}^{l s}=\omega\left(\rho \xi \sigma v, \sigma \sigma^{(1)} v, \sigma^{2}\right)_{0}^{T}, \quad C_{6, T}^{l s}=\omega\left(\sigma^{2} v, \rho \xi \sigma^{(1)} v, \sigma^{2}\right)_{0}^{T},
\end{aligned}
$$


$C_{10 d, T}^{l s}=\omega\left(\rho \xi \sigma v, \sigma^{2} v, \sigma \sigma^{(1)} v, \sigma^{2}\right)_{0}^{T}, \quad C_{10 e, T}^{l s}=\omega\left(\sigma^{2} v, \rho \xi \sigma v, \sigma \sigma^{(1)} v, \sigma^{2}\right)_{0}^{T}, \quad C_{10 f, T}^{l s}=\omega\left(\sigma^{2} v, \sigma \sigma^{(1)} v, \rho \xi \sigma v, \sigma^{2}\right)_{0}^{T}$.

Then using again Lemma 3.2 and (B.3)-(B.5) it comes:

$$
\begin{aligned}
& \frac{1}{2} \mathbb{E}\left[h^{(2)}\left(X_{T}^{P}\right) \int_{0}^{T}\left(\left(X_{t}^{P}-x_{0}\right)^{2}\left(\sigma_{t}^{(1)}\right)^{2} v_{t}+\left(X_{t}^{P}-x_{0}\right) V_{1, t} \sigma_{t} \sigma_{t}^{(1)}\right) \mathrm{d} t\right] \\
= & C_{3 a, T}^{l}\left[\mathcal{G}_{4}^{h}-\mathcal{G}_{3}^{h}+\frac{\mathcal{G}_{2}^{h}}{4}\right]+C_{2 a, T}^{l} \frac{\mathcal{G}_{2}^{h}}{2}+\left(C_{2, T}^{l s}+C_{3, T}^{l s}\right)\left[\frac{\mathcal{G}_{4}^{h}}{2}-\frac{\mathcal{G}_{3}^{h}}{4}\right]+C_{1, T}^{l s} \frac{\mathcal{G}_{2}^{h}}{2} .
\end{aligned}
$$

Finally in view of (B.8), we sum the contributions (B.12)-(B.13)-(B.14) to obtain

$$
\begin{aligned}
& \frac{1}{2} \mathbb{E}\left[h^{(2)}\left(X_{T}^{P}\right) X_{1, T}^{2}\right]-\frac{1}{8} \mathbb{E}\left[h^{(2)}\left(X_{T}^{P}\right) \int_{0}^{T} \frac{V_{1, t}^{2}}{v_{t}} \sigma_{t}^{2} \mathrm{~d} t\right] \\
= & \left(2 C_{9 a, T}^{l}+C_{9 b, T}^{l}\right)\left[\mathcal{G}_{6}^{h}-3 \mathcal{G}_{5}^{h}+\frac{13 \mathcal{G}_{4}^{h}}{4}-\frac{3 \mathcal{G}_{3}^{h}}{2}+\frac{\mathcal{G}_{2}^{h}}{4}\right] \\
& +C_{4, T}^{l}\left[2 \mathcal{G}_{4}^{h}-\frac{7 \mathcal{G}_{3}^{h}}{2}+\frac{3 \mathcal{G}_{2}^{h}}{2}\right]+C_{4, T}^{l s}\left[\mathcal{G}_{4}^{h}-\frac{3 \mathcal{G}_{3}^{h}}{2}+\frac{\mathcal{G}_{2}^{h}}{2}\right] \\
& +\left(C_{10 a, T}^{l s}+C_{10 b, T}^{l s}+C_{10 c, T}^{l s}+C_{10 d, T}^{l s}+C_{10 e, T}^{l s}+C_{10 f, T}^{l s}\right)\left[\frac{\mathcal{G}_{6}^{h}}{2}-\frac{5 \mathcal{G}_{5}^{h}}{4}+\mathcal{G}_{4}^{h}-\frac{\mathcal{G}_{3}^{h}}{4}\right] \\
& +C_{5, T}^{l s}\left[\frac{\mathcal{G}_{4}^{h}}{2}-\mathcal{G}_{3}^{h}+\frac{\mathcal{G}_{2}^{h}}{2}\right]+C_{3, T}^{s}\left[\frac{\mathcal{G}_{4}^{h}}{4}-\frac{\mathcal{G}_{3}^{h}}{2}+\frac{\mathcal{G}_{2}^{h}}{4}\right]+C_{2, T}^{s}\left[\frac{\mathcal{G}_{4}^{h}}{4}-\frac{\mathcal{G}_{3}^{h}}{4}\right] \\
& +\left(2 C_{4 a, T}^{s}+C_{4 b, T}^{s}\right)\left[\frac{\mathcal{G}_{6}^{h}}{4}-\frac{\mathcal{G}_{5}^{h}}{2}+\frac{\mathcal{G}_{4}^{h}}{4}\right]+C_{6, T}^{l s}\left[\frac{\mathcal{G}_{4}^{h}}{2}-\frac{3 \mathcal{G}_{3}^{h}}{4}+\frac{\mathcal{G}_{2}^{h}}{4}\right] \\
& +C_{3 a, T}^{l}\left[\mathcal{G}_{4}^{h}-\mathcal{G}_{3}^{h}+\frac{\mathcal{G}_{2}^{h}}{4}\right]+C_{2 a, T}^{l} \frac{\mathcal{G}_{2}^{h}}{2}+\left(C_{2, T}^{l s}+C_{3, T}^{l s}\right)\left[\frac{\mathcal{G}_{4}^{h}}{2}-\frac{\mathcal{G}_{3}^{h}}{4}\right]+C_{1, T}^{l s} \frac{\mathcal{G}_{2}^{h}}{2} .
\end{aligned}
$$

$\triangleright$ Step 4: some mathematical reductions. There are some relations between the expansion coefficients. Namely, for any integrable functions $f_{1}, f_{2}, g_{1}, g_{2}:[0, T] \mapsto \mathbb{R}$, we have

$$
\begin{gathered}
\omega\left(f_{1}, f_{2}\right)_{0}^{T} \omega\left(g_{1}, g_{2}\right)_{0}^{T}=\omega\left(f_{1}, f_{2}, g_{1}, g_{2}\right)_{0}^{T}+\omega\left(f_{1}, g_{1}, g_{2}, f_{2}\right)_{0}^{T}+\omega\left(f_{1}, g_{1}, f_{2}, g_{2}\right)_{0}^{T} \\
+\omega\left(g_{1}, g_{2}, f_{1}, f_{2}\right)_{0}^{T}+\omega\left(g_{1}, f_{1}, f_{2}, g_{2}\right)_{0}^{T}+\omega\left(g_{1}, f_{1}, g_{2}, f_{2}\right)_{0}^{T} .
\end{gathered}
$$

This relation is proved by standard integral manipulations. It gives:

$$
\begin{aligned}
\frac{\left(C_{1, T}^{s}\right)^{2}}{2} & =2 C_{4 a, T}^{s}+C_{4 b, T}^{s}, & \frac{\left(C_{1, T}^{l}\right)^{2}}{2}=2 C_{9 a, T}^{l}+C_{9 b, T}^{l}, \\
C_{1, T}^{l} C_{1, T}^{s} & =C_{10 a, T}^{l s}+C_{10 b, T}^{l s}+C_{10 c, T}^{l s}+C_{10 d, T}^{l s}+C_{10 e, T}^{l s}+C_{10 f, T}^{l s} . &
\end{aligned}
$$

In addition we set $C_{2, T}^{l}=C_{2 a, T}^{l}+C_{2 b, T}^{l}$ and $C_{3, T}^{l}=C_{3 a, T}^{l}+C_{3 b, T}^{l}$.

$\triangleright$ Final step. Taking advantage of the above simplifications and summing up the different contributions (B.2)-(B.7)-(B.15) of steps $1-2-3$, we obtain the announced formula (2.3), gathering the corrective terms according to the order of the Greeks.

Appendix C. Applications of the implied volatility expansion at mid-point for timeindependent local and stochastic volatility models with CIR-type variance. We specify in this section the form of the implied volatility approximation at mid point when considering the time-independent local and stochastic volatility model with CIR-type variance:

$$
\mathrm{d} X_{t}=\sigma\left(X_{t}\right) \sqrt{Y_{t}}\left[d W_{t}-\frac{\sigma\left(X_{t}\right) \sqrt{Y_{t}}}{2} \mathrm{~d} t\right], \quad X_{0}=x_{0}
$$




$$
\begin{aligned}
\mathrm{d} Y_{t} & =\kappa\left(\theta-Y_{t}\right) \mathrm{d} t+\xi \sqrt{Y_{t}} \mathrm{~d} B_{t}, \quad Y_{0}=v_{0}, \\
\mathrm{~d}\langle W, B\rangle_{t} & =\rho \mathrm{d} t .
\end{aligned}
$$

Note that this model is equivalent to the time-dependent model (1.2)-(1.3) by setting $V_{t}=Y_{t} e^{\kappa t}$, $\sigma(t, x)=\sigma(x) e^{-\kappa t / 2}, \alpha_{t}=e^{\kappa t} \kappa \theta, \xi_{t}=\xi e^{\kappa t / 2}$. Thus we can apply our different price and implied volatility expansion theorems by considering in the various corrective coefficients $C$ (defined in Theorem 2.4 and Definitions 4.1 and 4.3) the above time dependent functions. These coefficients are obtained by simple iterated integrations of exponential functions. Using Mathematica, we derive the following explicit expressions.

Proposition C.1. For $\sigma(t, x)=\sigma(x) e^{-\frac{\kappa t}{2}}, v_{t}=v_{0}+\theta\left(e^{\kappa t}-1\right), \xi_{t}=\xi e^{\frac{\kappa t}{2}}$ and $\rho_{t}=\rho$, one has:

$$
\begin{array}{rlrl}
\int_{0}^{T} v_{t} e^{-\kappa t} \mathrm{~d} t & =\bar{v} T, & & C_{1, T}^{l}(x)=\frac{\sigma^{3}(x) \sigma^{(1)}(x) \bar{v}^{2} T^{2}}{2}, \\
C_{2, T}^{l}(x) & =\frac{\sigma^{2}(x)\left[\left(\sigma^{(1)}\right)^{2}+\sigma \sigma^{(2)}\right](x) \bar{v}^{2} T^{2}}{2}, & & C_{3, T}^{l}(x)=C_{5, T}^{l}(x)=\frac{\sigma^{4}(x)\left[\left(\sigma^{(1)}\right)^{2}+\sigma \sigma^{(2)}\right](x) \bar{v}^{3} T^{3}}{6}, \\
C_{4, T}^{l}(x) & =\frac{\sigma^{4}(x)\left(\sigma^{(1)}\right)^{2}(x) \bar{v}^{3} T^{3}}{6}, & C_{6, T}^{l}(x) & =\frac{\sigma^{6}(x)\left(\sigma^{(1)}\right)^{2}(x) \bar{v}^{4} T^{4}}{24}, \\
C_{7, T}^{l}(x) & =\left[\left(\sigma^{(1)}\right)^{2}+\sigma \sigma^{(2)}\right](x) \bar{v} T, & C_{8, T}^{l}(x)=\frac{\left[\sigma \sigma^{(1)}\right]^{2}(x) \bar{v}^{2} T^{2}}{2}, \\
C_{1, T}^{s}(x) & =\frac{\rho \xi \sigma^{3}(x) R_{1}^{s} T^{2}}{2}, & C_{2, T}^{s}(x)=\frac{\rho^{2} \xi^{2} \sigma^{4}(x) R_{2}^{s} T^{3}}{6}, \\
C_{3, T}^{s}(x) & =\frac{\xi^{2} \sigma^{4}(x) R_{3}^{s} T^{3}}{6}, & C_{1, T}^{l s}(x)=C_{8, T}^{l s}(x)=\frac{\rho \xi \sigma^{2}(x) \sigma^{(1)}(x) R_{1}^{s} T^{2}}{2}, \\
C_{2, T}^{l s}(x) & =C_{5, T}^{l s}(x)=\frac{\rho \xi \sigma^{4}(x) \sigma^{(1)}(x) R_{1}^{l s} T^{3}}{6}, & C_{3, T}^{l s}(x)=C_{6, T}^{l s}(x)=C_{9, T}^{l s}(x)=\frac{\rho \xi \sigma^{4}(x) \sigma^{(1)}(x) R_{2}^{l s} T^{3}}{6}, \\
C_{4, T}^{l s}(x) & =C_{7, T}^{l s}(x)=\frac{\rho \xi \sigma^{4}(x) \sigma^{(1)}(x) R_{3}^{l s} T^{3}}{6}, &
\end{array}
$$

where

$$
\begin{aligned}
\bar{v}= & \left(v_{0}-\theta\right) \frac{e^{-\kappa T}\left(-1+e^{\kappa T}\right)}{\kappa T}+\theta \\
R_{1}^{s}= & \left(v_{0}-\theta\right) \frac{e^{-\kappa T}\left(-2 \kappa T+2 e^{\kappa T}-2\right)}{\kappa^{2} T^{2}}+\theta \frac{e^{-\kappa T}\left(2 \kappa T e^{\kappa T}-2 e^{\kappa T}+2\right)}{\kappa^{2} T^{2}}, \\
R_{2}^{s}= & \left(v_{0}-\theta\right) \frac{e^{-\kappa T}\left(-3 \kappa T(\kappa T+2)+6 e^{\kappa T}-6\right)}{\kappa^{3} T^{3}}+\theta \frac{e^{-\kappa T}\left(6 e^{\kappa T}(\kappa T-2)+6 \kappa T+12\right)}{\kappa^{3} T^{3}}, \\
R_{3}^{s}= & \left(v_{0}-\theta\right) \frac{e^{-2 \kappa T}\left(-6 e^{\kappa T} \kappa T+3 e^{2 \kappa T}-3\right)}{\kappa^{3} T^{3}}+\theta \frac{e^{-2 \kappa T}\left(12 e^{\kappa T}+3 e^{2 \kappa T}(2 \kappa T-3)-3\right)}{2 \kappa^{3} T^{3}}, \\
R_{1}^{l s}= & \frac{3}{2 \kappa^{3} T^{3}}\left\{e^{-2 \kappa T}\left(v_{0}-\theta\right)\left(v_{0}(3+2 \kappa T)-\theta(5+2 \kappa T)\right)+2 e^{-\kappa T}\left(\theta^{2}(4+\kappa T(6+\kappa T))\right.\right. \\
& \left.\left.-\theta v_{0}(-2+\kappa T(4+\kappa T))-2 v_{0}^{2}\right)+\theta^{2}(4 \kappa T-13)+4 \theta v_{0}+v_{0}^{2}\right\}, \\
R_{2}^{l s}= & \frac{3}{\kappa^{3} T^{3}}\left\{-e^{-2 \kappa T}\left(v_{0}-\theta\right)^{2}+e^{-\kappa T}\left(\theta^{2}(-4+\kappa T(-2+\kappa T))-\theta v_{0}(-2+\kappa T(-4+\kappa T))-2 \kappa T v_{0}^{2}\right)\right. \\
& \left.+\theta^{2}(5+\kappa T(-4+\kappa T))+2 \theta v_{0}(-2+\kappa T)+v_{0}^{2}\right\}, \\
R_{3}^{l s}= & \frac{3}{2 \kappa^{3} T^{3}}\left\{e^{-2 \kappa T}\left(v_{0}-\theta\right)\left(v_{0}(3+2 \kappa T)-\theta(5+2 \kappa T)\right)-4 e^{-\kappa T}\left(v_{0}-2 \theta\right)^{2}\right.
\end{aligned}
$$




$$
\left.+\theta^{2}(11+2 \kappa T(-4+\kappa T))+4 \theta v_{0}(-2+\kappa T)+v_{0}^{2}\right\} .
$$

We have in addition the relation: $\bar{v} T \frac{R_{1}^{s} T^{2}}{2}=\frac{\left(R_{1}^{l s}+R_{2}^{l s}+R_{3}^{l s}\right) T^{3}}{6}$.

After some simplifications, we obtain the following expressions for the coefficients $\gamma$ and $\pi$ defined in Definitions 4.1 and 4.3.

Proposition C.2. For $\sigma(t, x)=\sigma(x) e^{-\frac{\kappa t}{2}}, v_{t}=v_{0}+\theta\left(e^{\kappa t}-1\right), \xi_{t}=\xi e^{\frac{\kappa t}{2}}$ and $\rho_{t}=\rho$, one has:

$$
\begin{aligned}
\gamma_{0 a, T}\left(x_{0}\right)= & \sigma\left(x_{0}\right) \sqrt{\bar{v}}\left\{1+\frac{\rho \xi \sigma\left(x_{0}\right) R_{1}^{s} T}{8 \bar{v}}\right\} \\
\gamma_{1 a, T}\left(x_{0}\right)= & \sigma\left(x_{0}\right) \sqrt{\bar{v}}\left\{-\frac{\sigma^{(1)}\left(x_{0}\right)}{2 \sigma\left(x_{0}\right)}-\frac{\rho \xi R_{1}^{s}}{4 \sigma\left(x_{0}\right) \bar{v}^{2}}\right\} \\
\gamma_{0 b, T}\left(x_{0}\right)= & \sigma\left(x_{0}\right) \sqrt{\bar{v}}\left\{\bar{v} T\left[\frac{\sigma\left(x_{0}\right) \sigma^{(2)}\left(x_{0}\right)}{12}-\left(\sigma^{(1)}\right)^{2}\left(x_{0}\right)\left(\frac{1}{24}+\frac{\sigma^{2}\left(x_{0}\right) \bar{v} T}{96}\right)\right]\right. \\
& +\frac{\rho^{2} \xi^{2} T}{\bar{v}^{2}}\left[\frac{3\left(R_{1}^{s}\right)^{2}}{32 \bar{v}}+R_{2}^{s}\left(\frac{\sigma^{2}\left(x_{0}\right) \bar{v} T}{48}-\frac{1}{12}\right)\right]-\frac{\xi^{2} T R_{3}^{s}}{\bar{v}^{2}}\left[\frac{1}{24}+\frac{\sigma^{2}\left(x_{0}\right) \bar{v} T}{96}\right] \\
& \left.+\frac{\rho \xi \sigma^{(1)}\left(x_{0}\right) T}{\bar{v}}\left[\frac{R_{1}^{s}}{8}+\sigma^{2}\left(x_{0}\right) \bar{v} T\left(\frac{R_{2}^{l s}}{48 \bar{v}}-\frac{R_{1}^{s}}{32}\right)\right]\right\} \\
\gamma_{1 b, T}\left(x_{0}\right)= & \sigma\left(x_{0}\right) \sqrt{\bar{v}}\left\{\frac{\rho^{2} \xi^{2} T}{\bar{v}^{2}}\left[\frac{3\left(R_{1}^{s}\right)^{2}}{32 \bar{v}}-\frac{R_{2}^{s}}{12}\right]-\frac{\rho \xi \sigma^{(1)}\left(x_{0}\right) T}{\bar{v}}\left[\frac{R_{1}^{s}}{16}+\frac{R_{2}^{l s}}{24 \bar{v}}\right]\right\}, \\
\gamma_{2, T}\left(x_{0}\right)= & \sigma\left(x_{0}\right) \sqrt{\bar{v}}\left\{\frac{\sigma^{(2)}\left(x_{0}\right)}{6 \sigma\left(x_{0}\right)}-\frac{\left(\sigma^{(1)}\right)^{2}\left(x_{0}\right)}{12 \sigma^{2}\left(x_{0}\right)}+\frac{\rho^{2} \xi^{2}}{\sigma^{2}\left(x_{0}\right) \bar{v}^{3}}\left[\frac{R_{2}^{s}}{12}-\frac{3\left(R_{1}^{s}\right)^{2}}{16 \bar{v}}\right]+\frac{\xi^{2} R_{3}^{s}}{24 \sigma^{2}\left(x_{0}\right) \bar{v}^{3}}\right\}, \\
\pi_{1 a, T}\left(x_{a v g}\right)= & -\sigma\left(x_{a v g}\right) \sqrt{\bar{v}} \frac{\rho \xi R_{1}^{s}}{4 \sigma\left(x_{a v g}\right) \bar{v}^{2}}, \\
\pi_{1 b, T}\left(x_{a v g}\right)= & \sigma\left(x_{a v g}\right) \sqrt{\bar{v}}\left\{\frac{\rho^{2} \xi^{2} T}{\bar{v}^{2}}\left[\frac{3\left(R_{1}^{s}\right)^{2}}{32 \bar{v}}-\frac{R_{2}^{s}}{12}\right]+\frac{\rho \xi \sigma^{(1)}\left(x_{a v g}\right) T}{\bar{v}}\left[\frac{R_{1}^{s}}{16}-\frac{R_{2}^{l s}}{24 \bar{v}}\right]\right\}, \\
\pi_{2, T}\left(x_{a v g}\right)= & \sigma\left(x_{a v g}\right) \sqrt{\bar{v}}\left\{\frac{\sigma^{(2)}\left(x_{a v g}\right)}{24 \sigma\left(x_{a v g}\right)}-\frac{\left(\sigma^{(1)}\right)^{2}\left(x_{a v g}\right)}{12 \sigma^{2}\left(x_{a v g}\right)}+\frac{\rho^{2} \xi^{2}}{\sigma^{2}\left(x_{a v g}\right) \bar{v}^{3}}\left[\frac{R_{2}^{s}}{12}-\frac{3\left(R_{1}^{s}\right)^{2}}{16 \bar{v}}\right]+\frac{\xi^{2} R_{3}^{s}}{24 \sigma^{2}\left(x_{a v g}\right) \bar{v}^{3}}\right\}
\end{aligned}
$$

\section{REFERENCES}

[AE08] E. Alòs and C.O. Ewald. Malliavin differentiability of the Heston volatility and applications to option pricing. Advances in Applied Probability, 40(1):144-162, 2008.

[AP07] L.B.G. Andersen and V.V. Piterbarg. Moment explosions in stochastic volatility models. Finance and Stochastics, 11:29-50, 2007.

[BBF04] H. Berestycki, J. Busca, and I. Florent. Computing the implied volatility in stochastic volatility models. Comm. Pure Appl. Math., 10:1352-1373, 2004.

[BG12] R. Bompis and E. Gobet. Asymptotic and non asymptotic approximations for option valuation. In T. Gerstner and P. Kloeden, editors, Recent Developments in Computational Finance: Foundations, Algorithms and Applications. World Scientific Publishing Company, 2012.

[BGM09] E. Benhamou, E. Gobet, and M. Miri. Smart expansion and fast calibration for jump diffusion. Finance and Stochastics, 13(4):563-589, 2009.

[BGM10a] E. Benhamou, E. Gobet, and M. Miri. Expansion formulas for European options in a local volatility model. International Journal of Theoretical and Applied Finance, 13(4):603-634, 2010.

[BGM10b] E. Benhamou, E. Gobet, and M. Miri. Time dependent Heston model. SIAM Journal on Financial Mathematics, 1:289-325, 2010.

[Bom13] R. Bompis. Stochastic expansion for the diffusion processes and applications to option pricing. PhD thesis, Ecole Polytechnique, http://pastel.archives-ouvertes.fr/pastel-00921808, 2013. 
[De 11] S. De Marco. Smoothness and asymptotic estimates of densities for SDEs with locally smooth coefficients and applications to square root-type diffusions. Ann. Appl. Probab., 21(4):1282-1321, 2011.

[EKO11] B. Engelmann, F. Koster, and D. Oeltz. Calibration of the Heston local stochastic volatility model: a finite volume scheme. Preprint available at http://www. quantsolutions. de/downloads/ HestonL V. pdf, 2011.

$\left[\mathrm{FGG}^{+}{ }^{15}\right] \quad$ P.K. Friz, J. Gatheral, A. Gulisashvili, A. Jacquier, and J. Teichmann, editors. Large Deviations and Asymptotic Methods in Finance. Springer Proceedings in Mathematics and Statistics. SpringerVerlag, Berlin, 2015.

[FJ09] M. Forde and A. Jacquier. Small-time asymptotics for implied volatility under the Heston model. International Journal of Theoretical and Applied Finance, 12(6):861-876, 2009.

[FJ11] M. Forde and A. Jacquier. Small-time asymptotics for an uncorrelated local-stochastic volatility model. Applied Mathematical Finance, 8(6):517-535, 2011.

[FL11] J.P. Fouque and M. Lorig. A fast mean-reverting correction to Heston stochastic volatility model. SIAM Journal on Financial Mathematics, 2:221-254, 2011.

[Fou08] N. Fournier. Smoothness of the law of some one-dimensional jumping S.D.E.s with non-constant rate of jump. Electron. J. Probab., 13(6):135-156, 2008.

[FP13] M. Forde and A. Pogudin. The large-maturity smile for the SABR and CEV-Heston models. International Journal of Theoretical and Applied Finance, 16:1350047, 2013.

[FPSK11] J.P. Fouque, G. Papanicolaou, R. Sircar, and S. Knut. Multiscale stochastic volatility for equity, interest rate, and credit derivatives. Cambridge University Press, Cambridge, 2011.

[FPSS04] J-P. Fouque, G. Papanicolaou, R. Sircar, and K. Solna. Maturity cycles in implied volatility. Finance and Stochastics, 8(4):451-477, 2004.

[Gla03] P. Glasserman. Monte Carlo methods in Financial Engineering. Springer Verlag, New York, 2003.

[GM14] E. Gobet and M. Miri. Weak approximation of averaged diffusion processes. Stochastic Processes and their Applications, 124:475-504, 2014.

[GP14] E. Gobet and S. Pagliarani. Analytical approximations of BSDEs with non-smooth driver. In revision for SIAM Financial Mathematics, 2014.

[Gul12] A. Gulisashvili. Analytically tractable stochastic stock price models. Springer Finance, 2012.

[Hen08] P. Henry-Labordère. Analysis, Geometry, and Modeling in Finance: Advanced Methods in Option Pricing. Chapman and Hall, 2008.

[HKLW02] P.S. Hagan, D. Kumar, A.S. Lesniewski, and D.E. Woodward. Managing smile risk. Willmott Magazine, pages $84-108,2002$.

[JT11] R. Jordan and C. Tier. Asymptotic approximations to deterministic and stochastic volatility models. SIAM J. Fin. Math., 2(1):935-964, 2011.

[KS91] I. Karatzas and S.E. Shreve. Brownian motion and stochastic calculus. Springer Verlag, second edition, 1991.

[Lew00] A.L. Lewis. Option valuation under stochastic volatility: with Mathematica code. Finance Press, Newport Beach, California, 2000.

[Lew07] A.L. Lewis. Geometries and smile asymptotics for a class of stochastic volatility models. www.optioncity.net, 2007.

[LPP14] M. Lorig, S. Pagliarani, and A. Pascucci. Explicit implied vols for multifactor local-stochastic vol models. Available at SSRN 2283874, 2014.

[Nua06] D. Nualart. Malliavin calculus and related topics. Springer-Verlag, Berlin, second edition, 2006. (With corrections on the webpage of the author).

[PP13] S. Pagliarani and A. Pascucci. Local stochastic volatility with jumps: analytical approximations. Int. J. Theor. Appl. Finance, 16(8):1350050, 2013.

[Pro04] P. Protter. Stochastic integration and differential equations. Springer Verlag, second edition, 2004.

[TY12] A. Takahashi and T. Yamada. An asymptotic expansion with push-down of Malliavin weights. SIAM J. Financ. Math., 3(1):95-136, 2012. 